\title{
Hybrid Extragradient Method with Regularization for Convex Minimization, Generalized Mixed Equilibrium, Variational Inequality and Fixed Point Problems
}

\author{
Lu-Chuan Ceng ${ }^{1}$ and Juei-Ling $\mathbf{H o}^{2}$ \\ ${ }^{1}$ Department of Mathematics, Shanghai Normal University and Scientific Computing Key Laboratory of Shanghai Universities, \\ Shanghai 200234, China \\ ${ }^{2}$ Department of Finance, Tainan University of Technology, No. 529, Zhongzheng Road, YongKang District, Tainan 71002, Taiwan
}

Correspondence should be addressed to Juei-Ling Ho; t20054@mail.tut.edu.tw

Received 15 October 2013; Revised 25 November 2013; Accepted 25 November 2013; Published 4 February 2014

Academic Editor: Erdal Karapınar

Copyright (C) 2014 L.-C. Ceng and J.-L. Ho. This is an open access article distributed under the Creative Commons Attribution License, which permits unrestricted use, distribution, and reproduction in any medium, provided the original work is properly cited.

\begin{abstract}
We introduce two iterative algorithms by the hybrid extragradient method with regularization for finding a common element of the set of solutions of the minimization problem for a convex and continuously Fréchet differentiable functional, the set of solutions of finite generalized mixed equilibrium problems, the set of solutions of finite variational inequalities for inverse strong monotone mappings and the set of fixed points of an asymptotically $\kappa$-strict pseudocontractive mapping in the intermediate sense in a real Hilbert space. We prove some strong and weak convergence theorems for the proposed iterative algorithms under mild conditions.
\end{abstract}

\section{Introduction}

Throughout this paper, we assume that $H$ is a real Hilbert space with inner product $\langle\cdot, \cdot\rangle$ and norm $\|\cdot\|$; let $C$ be a nonempty closed convex subset of $H$ and let $P_{C}$ be the metric projection of $H$ onto $C$. Let $S: C \rightarrow C$ be a self-mapping on $C$. We denote by $\operatorname{Fix}(S)$ the set of fixed points of $S$ and by $\mathbf{R}$ the set of all real numbers.

Let $\varphi: C \rightarrow \mathbf{R}$ be a real-valued function, let $A:$ $C \rightarrow H$ be a nonlinear mapping, and let $F: C \times C \rightarrow \mathbf{R}$ be a bifunction. In 2008, Peng and Yao [1] introduced the following generalized mixed equilibrium problem (GMEP) of finding $x \in C$ such that

$$
F(x, y)+\varphi(y)-\varphi(x)+\langle A x, y-x\rangle \geq 0, \quad \forall y \in C .
$$

We denote the set of solutions of GMEP (1) by $\operatorname{GMEP}(F, \varphi, A)$. The GMEP (1) is very general in the sense that it includes, as special cases, optimization problems, variational inequalities, minimax problems, and Nash equilibrium problems in noncooperative games. The GMEP is further considered and studied in [2-5].
Let $f: C \rightarrow \mathbf{R}$ be a convex and continuously Fréchet differentiable functional. Consider the convex minimization problem (CMP) of minimizing $f$ over the constraint set $C$

$$
\min _{x \in C} f(x)
$$

(assuming the existence of minimizers). We denote by $\Gamma$ the set of minimizers of CMP (2). The gradient-projection algorithm (GPA) generates a sequence $\left\{x_{n}\right\}$ determined by the gradient $\nabla f$ and the metric projection $P_{C}$ as follows:

$$
x_{n+1}:=P_{C}\left(x_{n}-\lambda \nabla f\left(x_{n}\right)\right), \quad \forall n \geq 0,
$$

or more generally,

$$
x_{n+1}:=P_{C}\left(x_{n}-\lambda_{n} \nabla f\left(x_{n}\right)\right), \quad \forall n \geq 0,
$$

where, in both (3) and (3)', the initial guess $x_{0}$ is taken from $C$ arbitrarily and the parameters $\lambda$ or $\lambda_{n}$ are positive real numbers. The convergence of algorithms (3) and (3)' depends on the behavior of the gradient $\nabla f$.

Since the Lipschitz continuity of the gradient $\nabla f$ implies that it is actually $(1 / L)$-inverse strongly monotone (ism) [6], 
its complement can be an averaged mapping (i.e., it can be expressed as a proper convex combination of the identity mapping and a nonexpansive mapping). Consequently, the GPA can be rewritten as the composite of a projection and an averaged mapping, which is again an averaged mapping. This shows that averaged mappings play an important role in the GPA. Recently, Xu [7] used averaged mappings to study the convergence analysis of the GPA, which is hence an operatororiented approach.

Assume that the CMP (2) is consistent and the gradient $\nabla f$ is $L$-Lipschitz continuous with $L>0$. Let $Q: C \rightarrow C$ be a $\rho$-contraction with $\rho \in[0,1)$. Xu [7] introduced the following hybrid GPA:

$$
x_{n+1}=\theta_{n} Q x_{n}+\left(1-\theta_{n}\right) P_{C}\left(x_{n}-\lambda_{n} \nabla f\left(x_{n}\right)\right), \quad \forall n \geq 0,
$$

where $\left\{\theta_{n}\right\} \subset[0,1]$ and $0<\liminf _{n \rightarrow \infty} \lambda_{n} \leq$ $\limsup _{n \rightarrow \infty} \lambda_{n}<2 / L$. It was proven that under appropriate conditions the sequence $\left\{x_{n}\right\}$ converges in norm to a minimizer of CMP (2); see [7, Theorem 5.2].

It is worth emphasizing that the regularization, in particular the traditional Tikhonov regularization, is usually used to solve ill-posed optimization problems. Consider the regularized minimization problem

$$
\min _{x \in C} f_{\alpha}(x):=f(x)+\frac{\alpha}{2}\|x\|^{2}
$$

where $\alpha>0$ is the regularization parameter and again $f$ is convex with $L$-Lipschitz continuous gradient $\nabla f$. In [7], Xu introduced another hybrid GPA with regularization

$$
\begin{aligned}
x_{n+1} & =P_{C}\left(I-\lambda_{n} \nabla f_{\alpha_{n}}\right) x_{n} \\
& =P_{C}\left(I-\lambda_{n}\left(\nabla f+\alpha_{n} I\right)\right) x_{n}, \quad \forall n \geq 0,
\end{aligned}
$$

where (i) $0<\lambda_{n} \leq \alpha_{n} /\left(L+\alpha_{n}\right)^{2}$ for all $n \geq 0$; (ii) $\alpha_{n} \rightarrow 0$ (and $\lambda_{n} \rightarrow 0$ ) as $n \rightarrow \infty$; (iii) $\sum_{n=0}^{\infty} \alpha_{n} \lambda_{n}=\infty$; and (iv) $\left(\left|\lambda_{n}-\lambda_{n-1}\right|+\left|\alpha_{n} \lambda_{n}-\alpha_{n-1} \lambda_{n-1}\right|\right) /\left(\alpha_{n}^{2} \lambda_{n}^{2}\right) \rightarrow 0$ as $n \rightarrow \infty$. It was proven that $\left\{x_{n}\right\}$ converges strongly to the minimumnorm solution $x^{\dagger} \in \Gamma$ of CMP (2); see [7, Theorem 6.1]. Very recently, the hybrid GPA with regularization is extended to develop new extragradient methods with regularization in Ceng et al. [8, 9] for finding a common solution of the split feasibility problem (SFP) and the fixed point problem of a nonexpansive mapping in a real Hilbert space.

On the other hand, consider the following variational inequality problem (VIP): find a $\bar{x} \in C$ such that

$$
\langle A \bar{x}, y-\bar{x}\rangle \geq 0, \quad \forall y \in C .
$$

The solution set of VIP (7) is denoted by $\operatorname{VI}(C, A)$.

The VIP (7) was first discussed by Lions [10] and now is well known; there are a lot of different approaches towards solving VIP (7) in finite-dimensional and infinitedimensional spaces, and the research is intensively continued. The VIP (7) has many applications in computational mathematics, mathematical physics, operations research, mathematical economics, optimization theory, and other fields; see, for example, [11-14]. It is well known that if $A$ is a strongly monotone and Lipschitz-continuous mapping on $C$, then VIP (7) has a unique solution. Not only the existence and uniqueness of solutions are important topics in the study of VIP (7), but also how to actually find a solution of VIP (7) is important.

Motivated by the idea of Korpelevič's extragradient method [15], Nadezhkina and Takahashi [16] introduced an extragradient iterative scheme:

$$
\begin{gathered}
x_{0}=x \in C \text { chosen arbitrary, } \\
y_{n}=P_{C}\left(x_{n}-\lambda_{n} A x_{n}\right), \\
x_{n+1}=\alpha_{n} x_{n}+\left(1-\alpha_{n}\right) S P_{C}\left(x_{n}-\lambda_{n} A y_{n}\right), \quad \forall n \geq 0,
\end{gathered}
$$

where $A: C \rightarrow H$ is a monotone, $L$-Lipschitz continuous mapping, $S: C \rightarrow C$ is a nonexpansive mapping, and $\left\{\lambda_{n}\right\} \subset[a, b]$ for some $a, b \in(0,1 / L)$ and $\left\{\alpha_{n}\right\} \subset[c, d]$ for some $c, d \in(0,1)$. They proved the weak convergence of $\left\{x_{n}\right\}$ to an element of $\operatorname{Fix}(S) \cap \operatorname{VI}(C, A)$. Recently, inspired by Nadezhkina and Takahashi's iterative scheme [16], Zeng and Yao [17] introduced another iterative scheme for finding an element of $\operatorname{Fix}(S) \cap \operatorname{VI}(C, A)$ and derived the weak convergence result. Furthermore, by combining the CQ method and extragradient method, Nadezhkina and Takahashi [18] introduced an iterative process:

$$
\begin{gathered}
x_{0}=x \in C \text { chosen arbitrary, } \\
y_{n}=P_{C}\left(x_{n}-\lambda_{n} A x_{n}\right), \\
z_{n}=\alpha_{n} x_{n}+\left(1-\alpha_{n}\right) P_{C}\left(x_{n}-\lambda_{n} A y_{n}\right), \\
C_{n}=\left\{z \in C:\left\|z_{n}-z\right\| \leq\left\|x_{n}-z\right\|\right\}, \\
Q_{n}=\left\{z \in C:\left\langle x_{n}-z, x-x_{n}\right\rangle \geq 0\right\}, \\
x_{n+1}=P_{C_{n} \cap Q_{n}} x, \quad \forall n \geq 0 .
\end{gathered}
$$

They proved the strong convergence of $\left\{x_{n}\right\}$ to an element of $\operatorname{Fix}(S) \cap \operatorname{VI}(C, A)$ under appropriate conditions. Later on, Ceng and Yao [19] introduced an extragradient-like approximation method which is based on the above extragradient method and viscosity approximation method and derived a strong convergence result as well. Next, recall some concepts. A mapping $A: C \rightarrow H$ is called $L$-Lipschitz continuous if there exists a constant $L \geq 0$ such that

$$
\|A x-A y\| \leq L\|x-y\|, \quad \forall x, y \in C .
$$

In particular, if $L=1$, then $A$ is called a nonexpansive mapping; if $L \in[0,1)$, then $A$ is called a contraction.

Recall that a mapping $A: C \rightarrow H$ is called

(i) monotone if

$$
\langle A x-A y, x-y\rangle \geq 0, \quad \forall x, y \in C
$$

(ii) $\eta$-strongly monotone if there exists a constant $\eta>0$ such that

$$
\langle A x-A y, x-y\rangle \geq \eta\|x-y\|^{2}, \quad \forall x, y \in C ;
$$


(iii) $\alpha$-inverse-strongly monotone if there exists a constant $\alpha>0$ such that

$$
\langle A x-A y, x-y\rangle \geq \alpha\|A x-A y\|^{2}, \quad \forall x, y \in C .
$$

It is obvious that if $A$ is $\alpha$-inverse-strongly monotone, then $A$ is monotone and $(1 / \alpha)$-Lipschitz continuous.

Definition 1. Let $C$ be a nonempty subset of a normed space $X$ and let $S: C \rightarrow C$ be a self-mapping on $C$.

(i) $S$ is asymptotically nonexpansive (cf. [20]) if there exists a sequence $\left\{k_{n}\right\}$ of positive numbers satisfying the property $\lim _{n \rightarrow \infty} k_{n}=1$ and

$$
\left\|S^{n} x-S^{n} y\right\| \leq k_{n}\|x-y\|, \quad \forall n \geq 1, \forall x, y \in C
$$

(ii) $S$ is asymptotically nonexpansive in the intermediate sense [21] provided $S$ is uniformly continuous and

$$
\limsup _{n \rightarrow \infty} \sup _{x, y \in C}\left(\left\|S^{n} x-S^{n} y\right\|-\|x-y\|\right) \leq 0
$$

(iii) $S$ is uniformly Lipschitzian if there exists a constant $\mathscr{L}>0$ such that

$$
\left\|S^{n} x-S^{n} y\right\| \leq \mathscr{L}\|x-y\|, \quad \forall n \geq 1, \forall x, y \in C .
$$

It is clear that every nonexpansive mapping is asymptotically nonexpansive and every asymptotically nonexpansive mapping is uniformly Lipschitzian.

The class of asymptotically nonexpansive mappings was introduced by Goebel and Kirk [20] as an important generalization of the class of nonexpansive mappings. The existence of fixed points of asymptotically nonexpansive mappings was proved by Goebel and Kirk [20] as follows.

Theorem GK (see [20, Theorem 1]). IfC is a nonempty closed convex bounded subset of a uniformly convex Banach space, then every asymptotically nonexpansive mapping $S: C \rightarrow C$ has a fixed point in $C$.

The class of asymptotically nonexpansive mappings in the intermediate sense was introduced by Bruck et al. [21]. Recently, Kim and Xu [22] introduced the concept of asymptotically $\kappa$-strict pseudocontractive mappings in a Hilbert space as follows.

Definition 2. Let $C$ be a nonempty subset of a Hilbert space $H$. A mapping $S: C \rightarrow C$ is said to be an asymptotically $\kappa$ strict pseudocontractive mapping with sequence $\left\{\gamma_{n}\right\}$ if there exists a constant $\kappa \in[0,1)$ and a sequence $\left\{\gamma_{n}\right\}$ in $[0, \infty)$ with $\lim _{n \rightarrow \infty} \gamma_{n}=0$ such that

$$
\begin{aligned}
\left\|S^{n} x-S^{n} y\right\|^{2} \leq & \left(1+\gamma_{n}\right)\|x-y\|^{2} \\
& +\kappa\left\|x-S^{n} x-\left(y-S^{n} y\right)\right\|^{2},
\end{aligned}
$$

$\forall n \geq 1, \quad \forall x, y \in C$.
They studied weak and strong convergence theorems for this class of mappings. It is important to note that every asymptotically $\mathcal{\kappa}$-strict pseudocontractive mapping with sequence $\left\{\gamma_{n}\right\}$ is a uniformly $\mathscr{L}$-Lipschitzian mapping with $\mathscr{L}=\sup \left\{\left(\left(\kappa+\sqrt{1+(1-\kappa) \gamma_{n}}\right) /(1+\kappa)\right): n \geq 1\right\}$.

Recently, Sahu et al. [23] considered the concept of asymptotically $\kappa$-strict pseudocontractive mappings in the intermediate sense, which are not necessarily Lipschitzian.

Definition 3. Let $C$ be a nonempty subset of a Hilbert space $H$. A mapping $S: C \rightarrow C$ is said to be an asymptotically $\kappa$ strict pseudocontractive mapping in the intermediate sense with sequence $\left\{\gamma_{n}\right\}$ if there exist a constant $\kappa \in[0,1)$ and a sequence $\left\{\gamma_{n}\right\}$ in $[0, \infty)$ with $\lim _{n \rightarrow \infty} \gamma_{n}=0$ such that

$$
\begin{array}{r}
\limsup _{n \rightarrow \infty} \sup _{x, y \in C}\left(\left\|S^{n} x-S^{n} y\right\|^{2}-\left(1+\gamma_{n}\right)\|x-y\|^{2}\right. \\
\left.-\kappa\left\|x-S^{n} x-\left(y-S^{n} y\right)\right\|^{2}\right) \leq 0 .
\end{array}
$$

Put $c_{n}:=\max \left\{0, \sup _{x, y \in C}\left(\left\|S^{n} x-S^{n} y\right\|^{2}-\left(1+\gamma_{n}\right)\|x-y\|^{2}-\right.\right.$ $\left.\left.\kappa\left\|x-S^{n} x-\left(y-S^{n} y\right)\right\|^{2}\right)\right\}$. Then $c_{n} \geq 0(\forall n \geq 1), c_{n} \rightarrow$ $0(n \rightarrow \infty)$ and (17) reduces to the relation

$$
\begin{array}{r}
\left\|S^{n} x-S^{n} y\right\|^{2} \leq\left(1+\gamma_{n}\right)\|x-y\|^{2} \\
+\kappa\left\|x-S^{n} x-\left(y-S^{n} y\right)\right\|^{2}+c_{n}, \\
\forall n \geq 1, \quad \forall x, y \in C .
\end{array}
$$

Whenever $c_{n}=0$ for all $n \geq 1$ in (18), then $S$ is an asymptotically $\kappa$-strict pseudocontractive mapping with sequence $\left\{\gamma_{n}\right\}$. In 2009, Sahu et al. [23] derived the weak and strong convergence of the modified Mann iteration process for the class of asymptotically $\kappa$-strictly pseudocontractive mappings in the intermediate sense with sequence $\left\{\gamma_{n}\right\}$. More precisely, they established the following theorems.

Theorem SXY 1. Let $C$ be a nonempty closed convex subset of a real Hilbert space $H$ and let $S: C \rightarrow C$ be a uniformly continuous asymptotically $\kappa$-strict pseudocontractive mapping in the intermediate sense with sequence $\left\{\gamma_{n}\right\}$ such that $\operatorname{Fix}(S) \neq \emptyset$ and $\sum_{n=1}^{\infty} \gamma_{n}<\infty$. Assume that $\left\{\alpha_{n}\right\}$ is a sequence in $(0,1)$ such that $0<\delta \leq \alpha_{n} \leq 1-\kappa-\delta$ and $\sum_{n=1}^{\infty} \alpha_{n} c_{n}<\infty$. Let $\left\{x_{n}\right\}_{n=1}^{\infty}$ be a sequence in $C$ generated by the modified Mann iteration process:

$$
\begin{gathered}
x_{1}=x \in C \text { chosen arbitrary, } \\
y_{n}=\left(1-\alpha_{n}\right) x_{n}+\alpha_{n} S^{n} x_{n}, \\
C_{n}=\left\{z \in C:\left\|y_{n}-z\right\|^{2} \leq\left\|x_{n}-z\right\|^{2}+\theta_{n}\right\}, \\
Q_{n}=\left\{z \in C:\left\langle x_{n}-z, x-x_{n}\right\rangle \geq 0\right\}, \\
x_{n+1}=\left(1-\alpha_{n}\right) x_{n}+\alpha_{n} S^{n} x_{n}, \quad \forall n \geq 1 .
\end{gathered}
$$

Then $\left\{x_{n}\right\}$ converges weakly to an element of $\operatorname{Fix}(S)$. 
Theorem SXY 2. Let $C$ be a nonempty closed convex subset of a real Hilbert space $H$ and let $S: C \rightarrow C$ be a uniformly continuous asymptotically $\kappa$-strict pseudocontractive mapping in the intermediate sense with sequence $\left\{\gamma_{n}\right\}$ such that $\operatorname{Fix}(S)$ is nonempty and bounded. Let $\left\{\alpha_{n}\right\}$ be a sequence in $[0,1]$ such that $0<\delta \leq \alpha_{n} \leq 1-\kappa$ for all $n \geq 1$. Let $\left\{x_{n}\right\}$ be the sequence in $C$ generated by the following (CQ) algorithm:

$$
\begin{gathered}
x_{1}=x \in C \text { chosen arbitrary, } \\
y_{n}=\left(1-\alpha_{n}\right) x_{n}+\alpha_{n} S^{n} x_{n}, \\
C_{n}=\left\{z \in C:\left\|y_{n}-z\right\|^{2} \leq\left\|x_{n}-z\right\|^{2}+\theta_{n}\right\}, \\
Q_{n}=\left\{z \in C:\left\langle x_{n}-z, x-x_{n}\right\rangle \geq 0\right\}, \\
x_{n+1}=P_{C_{n} \cap Q_{n}} x, \quad \forall n \geq 1,
\end{gathered}
$$

where $\theta_{n}=c_{n}+\gamma_{n} \Delta_{n}$ and $\Delta_{n}=\sup \left\{\left\|x_{n}-z\right\|^{2}: z \in \operatorname{Fix}(S)\right\}<$ $\infty$. Then $\left\{x_{n}\right\}$ converges strongly to $P_{\mathrm{Fix}(S)} x$.

Subsequently, the iterative algorithms in Theorems SXY1 and SXY2 are extended to develop new iterative algorithms for finding a common solution of the VIP and the fixed point problem of an asymptotically strict pseudocontractive mapping in the intermediate sense in a real Hilbert space; see, for example, $[24,25]$.

On the other hand, Yao et al. [26] introduced two iterative algorithms for finding a common element of the set of fixed points of an asymptotically $\kappa$-strict pseudocontraction and the set of solutions of a mixed equilibrium problem in a real Hilbert space. Then they obtained some weak and strong convergence theorems for the proposed iterative algorithms. Very recently, motivated by Yao et al. [26], Cai and $\mathrm{Bu}$ [3] introduced two iterative algorithms for finding a common element of the set of solutions of finite generalized mixed equilibrium problems, the set of solutions of finite variational inequalities for inverse strong monotone mappings, and the set of fixed points of an asymptotically $\kappa$-strict pseudocontractive mapping in the intermediate sense in a real Hilbert space. Then they proved some strong and weak convergence theorems for the proposed iterative algorithms under appropriate conditions.

In this paper, inspired by the above facts, we introduce two iterative algorithms by hybrid extragradient method with regularization for finding a common element of the set of solutions of the CMP (2) for a convex functional $f$ : $C \rightarrow \mathbf{R}$ with $L$-Lipschitz continuous gradient $\nabla f$, the set of solutions of finite GMEPs, the set of solutions of finite VIPs for inverse strong monotone mappings, and the set of fixed points of an asymptotically $\kappa$-strict pseudocontractive mapping in the intermediate sense in a real Hilbert space. Then we prove some strong and weak convergence theorems for the proposed iterative algorithms under mild conditions. For recent related results, see, for example, $[7,24,27-31]$ and ther references therein.

\section{Preliminaries}

Let $H$ be a real Hilbert space whose inner product and norm are denoted by $\langle\cdot, \cdot\rangle$ and $\|\cdot\|$, respectively. Let $C$ be a nonempty closed convex subset of $H$. We write $x_{n} \rightarrow x$ to indicate that the sequence $\left\{x_{n}\right\}$ converges weakly to $x$ and $x_{n} \rightarrow x$ to indicate that the sequence $\left\{x_{n}\right\}$ converges strongly to $x$. Moreover, we use $\omega_{w}\left(x_{n}\right)$ to denote the weak $\omega$-limit set of the sequence $\left\{x_{n}\right\}$; that is,

$$
\begin{aligned}
\omega_{w}\left(x_{n}\right):= & \left\{x \in H: x_{n_{i}}-x\right. \\
& \left.\quad \text { for some subsequence }\left\{x_{n_{i}}\right\} \text { of }\left\{x_{n}\right\}\right\} .
\end{aligned}
$$

The metric (or nearest point) projection from $H$ onto $C$ is the mapping $P_{C}: H \rightarrow C$ which assigns to each point $x \in H$ the unique point $P_{C} x \in C$ satisfying the property

$$
\left\|x-P_{C} x\right\|=\inf _{y \in C}\|x-y\|=: d(x, C) .
$$

Some important properties of projections are gathered in the following proposition.

Proposition 4. For given $x \in H$ and $z \in C$ :

(i) $z=P_{C} x \Leftrightarrow\langle x-z, y-z\rangle \leq 0$, for all $y \in C$;

(ii) $z=P_{C} x \Leftrightarrow\|x-z\|^{2} \leq\|x-y\|^{2}-\|y-z\|^{2}$, for all $y \in$ $C$;

(iii) $\left\langle P_{C} x-P_{C} y, x-y\right\rangle \geq\left\|P_{C} x-P_{C} y\right\|^{2}$, for all $y \in H$.

Consequently, $P_{C}$ is nonexpansive and monotone.

If $A$ is an $\alpha$-inverse-strongly monotone mapping of $C$ into $H$, then it is obvious that $A$ is $(1 / \alpha)$-Lipschitz continuous. We also have that, for all $u, v \in C$ and $\lambda>0$,

$$
\begin{aligned}
\|(I- & \lambda A) u-(I-\lambda A) v \|^{2} \\
= & \|(u-v)-\lambda(A u-A v)\|^{2} \\
= & \|u-v\|^{2}-2 \lambda\langle A u-A v, u-v\rangle \\
& +\lambda^{2}\|A u-A v\|^{2} \\
\leq & \|u-v\|^{2}+\lambda(\lambda-2 \alpha)\|A u-A v\|^{2} .
\end{aligned}
$$

So if $\lambda \leq 2 \alpha$, then $I-\lambda A$ is a nonexpansive mapping from $C$ to $H$.

Definition 5. A mapping $T: H \rightarrow H$ is said to be

(a) nonexpansive if

$$
\|T x-T y\| \leq\|x-y\|, \quad \forall x, y \in H ;
$$

(b) firmly nonexpansive if $2 T-I$ is nonexpansive, or equivalently, if $T$ is 1-inverse strongly monotone (1ism),

$$
\langle x-y, T x-T y\rangle \geq\|T x-T y\|^{2}, \quad \forall x, y \in H ;
$$


alternatively, $T$ is firmly nonexpansive if and only if $T$ can be expressed as

$$
T=\frac{1}{2}(I+S),
$$

where $S: H \rightarrow H$ is nonexpansive; projections are firmly nonexpansive.

It can be easily seen that if $T$ is nonexpansive, then $I-T$ is monotone. It is also easy to see that a projection $P_{C}$ is 1 -ism. Inverse strongly monotone (also referred to as co-coercive) operators have been applied widely in solving practical problems in various fields.

Definition 6. A mapping $T: H \rightarrow H$ is said to be an averaged mapping if it can be written as the average of the identity $I$ and a nonexpansive mapping; that is,

$$
T \equiv(1-\alpha) I+\alpha S
$$

where $\alpha \in(0,1)$ and $S: H \rightarrow H$ is nonexpansive. More precisely, when the last equality holds, we say that $T$ is $\alpha$ averaged. Thus, firmly nonexpansive mappings (in particular, projections) are (1/2)-averaged maps.

Proposition 7 (see [32]). Let $T: H \rightarrow H$ be a given mapping.

(i) $T$ is nonexpansive if and only if the complement $I-T$ is $(1 / 2)$-ism.

(ii) If $T$ is $\nu$-ism, then for $\gamma>0, \gamma T$ is $(\nu / \gamma)$-ism.

(iii) $T$ is averaged if and only if the complement $I-T$ is $\nu$-ism for some $\nu>1 / 2$. Indeed, for $\alpha \in(0,1), T$ is $\alpha$-averaged if and only if $I-T$ is $(1 / 2 \alpha)$-ism.

Proposition 8 (see $[32,33])$. Let $S, T, V: H \rightarrow H$ be given operators.

(i) If $T=(1-\alpha) S+\alpha V$ for some $\alpha \in(0,1)$ and if $S$ is averaged and $V$ is nonexpansive, then $T$ is averaged.

(ii) $T$ is firmly nonexpansive if and only if the complement $I-T$ is firmly nonexpansive.

(iii) If $T=(1-\alpha) S+\alpha V$ for some $\alpha \in(0,1)$ and if $S$ is firmly nonexpansive and $V$ is nonexpansive, then $T$ is averaged.

(iv) The composite of finitely many averaged mappings is averaged. That is, if each of the mappings $\left\{T_{i}\right\}_{i=1}^{N}$ is averaged, then so is the composite $T_{1} \cdots T_{N}$. In particular, if $T_{1}$ is $\alpha_{1}$-averaged and $T_{2}$ is $\alpha_{2}$-averaged, where $\alpha_{1}, \alpha_{2} \in(0,1)$, then the composite $T_{1} T_{2}$ is $\alpha$ averaged, where $\alpha=\alpha_{1}+\alpha_{2}-\alpha_{1} \alpha_{2}$.

(v) If the mappings $\left\{T_{i}\right\}_{i=1}^{N}$ are averaged and have a common fixed point, then

$$
\bigcap_{i=1}^{N} \operatorname{Fix}\left(T_{i}\right)=\operatorname{Fix}\left(T_{1} \cdots T_{N}\right) .
$$

The notation $\operatorname{Fix}(T)$ denotes the set of all fixed points of the mapping T; that is, $\operatorname{Fix}(T)=\{x \in H: T x=x\}$.

We need some facts and tools in a real Hilbert space $H$ which are listed as lemmas below.

Lemma 9. Let $X$ be a real inner product space. Then there holds the following inequality:

$$
\|x+y\|^{2} \leq\|x\|^{2}+2\langle y, x+y\rangle, \quad \forall x, y \in X .
$$

Lemma 10. Let $\left\{x_{n}\right\}$ be a bounded sequence on a reflexive Banach space $X$. If $\omega_{w}\left(\left\{x_{n}\right\}\right)=\{x\}$, then $x_{n} \rightarrow x$.

Lemma 11. Let $A: C \rightarrow H$ be a monotone mapping. In the context of the variational inequality problem the characterization of the projection (see Proposition 4 (i)) implies

$$
u \in \mathrm{VI}(C, A) \Longleftrightarrow u=P_{C}(u-\lambda A u), \quad \text { forsome } \lambda>0 \text {. }
$$

Lemma 12. Let $H$ be a real Hilbert space. Then the following hold:

(i) $\|x-y\|^{2}=\|x\|^{2}-\|y\|^{2}-2\langle x-y, y\rangle$ for all $x, y \in H$;

(ii) $\|(1-t) x+t y\|^{2}=(1-t)\|x\|^{2}+t\|y\|^{2}-t(1-t)\|x-y\|^{2}$ for all $t \in[0,1]$ and for all $x, y \in H$;

(iii) If $\left\{x_{n}\right\}$ is a sequence in $H$ such that $x_{n} \rightarrow x$, it follows that

$\limsup _{n \rightarrow \infty}\left\|x_{n}-y\right\|^{2}=\limsup _{n \rightarrow \infty}\left\|x_{n}-x\right\|^{2}+\|x-y\|^{2}, \quad \forall y \in H$.

Lemma 13 ([23, Lemma 2.5]). Let $H$ be a real Hilbert space. Given a nonempty closed convex subset of $H$ and points $x, y, z \in H$ and given also a real number $a \in \mathbf{R}$, the set

$$
\left\{v \in C:\|y-v\|^{2} \leq\|x-v\|^{2}+\langle z, v\rangle+a\right\}
$$

is convex (and closed).

Lemma 14 ([23, Lemma 2.6]). Let $C$ be a nonempty subset of a Hilbert space $H$ and let $S: C \rightarrow C$ be an asymptotically $\kappa$-strict pseudocontractive mapping in the intermediate sense with sequence $\left\{\gamma_{n}\right\}$. Then

$$
\begin{aligned}
\| S^{n} x & -S^{n} y \| \\
\leq & \frac{1}{1-\kappa} \\
\quad & \times\left(\kappa\|x-y\| \sqrt{\left(1+(1-\kappa) \gamma_{n}\right)\|x-y\|^{2}+(1-\kappa) c_{n}}\right)
\end{aligned}
$$

for all $x, y \in C$ and $n \geq 1$.

Lemma 15 ([23, Lemma 2.7]). Let $C$ be a nonempty subset of a Hilbert space $H$ and let $S: C \rightarrow C$ be a uniformly continuous asymptotically $\kappa$-strict pseudocontractive mapping in the intermediate sense with sequence $\left\{\gamma_{n}\right\}$. Let $\left\{x_{n}\right\}$ be a sequence in $C$ such that $\left\|x_{n}-x_{n+1}\right\| \rightarrow 0$ and $\left\|x_{n}-S^{n} x_{n}\right\| \rightarrow 0$ as $n \rightarrow \infty$. Then $\left\|x_{n}-S x_{n}\right\| \rightarrow 0$ as $n \rightarrow \infty$. 
Lemma 16 (demiclosedness principle [23, Proposition 3.1]). Let $C$ be a nonempty closed convex subset of a Hilbert space $H$ and let $S: C \rightarrow C$ be a continuous asymptotically $\kappa$-strict pseudocontractive mapping in the intermediate sense with sequence $\left\{\gamma_{n}\right\}$. Then $I-S$ is demiclosed at zero in the sense that if $\left\{x_{n}\right\}$ is a sequence in $C$ such that $x_{n} \rightarrow x \in C$ and $\lim \sup _{m \rightarrow \infty} \lim \sup _{n \rightarrow \infty}\left\|x_{n}-S^{m} x_{n}\right\|=0$, then $(I-S) x=0$.

Lemma 17 ([23, Proposition 3.2]). Let $C$ be a nonempty closed convex subset of a Hilbert space $H$ and let $S: C \rightarrow$ $C$ be a continuous asymptotically $\kappa$-strict pseudocontractive mapping in the intermediate sense with sequence $\left\{\gamma_{n}\right\}$ such that $\operatorname{Fix}(S) \neq \emptyset$. Then $\operatorname{Fix}(S)$ is closed and convex.

Remark 18. Lemmas 16 and 17 give some basic properties of an asymptotically $\kappa$-strict pseudocontractive mapping in the intermediate sense with sequence $\left\{\gamma_{n}\right\}$. Moreover, Lemma 16 extends the demiclosedness principles studied for certain classes of nonlinear mappings in Kim and Xu [22], Górnicki [34], Marino and $\mathrm{Xu}$ [35], and $\mathrm{Xu}$ [36].

To prove a weak convergence theorem by a modified extragradient method with regularization for the CMP (2) and the fixed point problem of an asymptotically $\kappa$-strict pseudocontractive mapping in the intermediate sense, we need the following lemma due to Osilike et al. [37].

Lemma 19 (see [37, page. 80]). Let $\left\{a_{n}\right\}_{n=1}^{\infty},\left\{b_{n}\right\}_{n=1}^{\infty}$ and $\left\{\delta_{n}\right\}_{n=1}^{\infty}$ be sequences of nonnegative real numbers satisfying the inequality

$$
a_{n+1} \leq\left(1+\delta_{n}\right) a_{n}+b_{n}, \quad \forall n \geq 1 .
$$

If $\sum_{n=1}^{\infty} \delta_{n}<\infty$ and $\sum_{n=1}^{\infty} b_{n}<\infty$, then $\lim _{n \rightarrow \infty} a_{n}$ exists. If, in addition, $\left\{a_{n}\right\}_{n=1}^{\infty}$ has a subsequence which converges to zero, then $\lim _{n \rightarrow \infty} a_{n}=0$.

Corollary 20 (see [38, page. 303]). Let $\left\{a_{n}\right\}_{n=0}^{\infty}$ and $\left\{b_{n}\right\}_{n=0}^{\infty}$ be two sequences of nonnegative real numbers satisfying the inequality

$$
a_{n+1} \leq a_{n}+b_{n}, \quad \forall n \geq 0 .
$$

If $\sum_{n=0}^{\infty} b_{n}$ converges, then $\lim _{n \rightarrow \infty} a_{n}$ exists.

Recall that a Banach space $X$ is said to satisfy the Opial condition [39] if for any given sequence $\left\{x_{n}\right\} \subset X$ which converges weakly to an element $x \in X$, there holds the inequality

$$
\limsup _{n \rightarrow \infty}\left\|x_{n}-x\right\|<\limsup _{n \rightarrow \infty}\left\|x_{n}-y\right\|, \quad \forall y \in X, \quad y \neq x .
$$

It is well known in [39] that every Hilbert space $H$ satisfies the Opial condition.

Lemma 21 (see [24, Proposition 3.1]). Let $C$ be a nonempty closed convex subset of a real Hilbert space $H$ and let $\left\{x_{n}\right\}$ be a sequence in $H$. Suppose that

$$
\left\|x_{n+1}-p\right\|^{2} \leq\left(1+\lambda_{n}\right)\left\|x_{n}-p\right\|^{2}+\delta_{n}, \quad \forall p \in C, n \geq 1,
$$

where $\left\{\lambda_{n}\right\}$ and $\left\{\delta_{n}\right\}$ are sequences of nonnegative real numbers such that $\sum_{n=1}^{\infty} \lambda_{n}<\infty$ and $\sum_{n=1}^{\infty} \delta_{n}<\infty$. Then $\left\{P_{C} x_{n}\right\}$ converges strongly in $C$.

A set-valued mapping $T: H \rightarrow 2^{H}$ is called monotone if for all $x, y \in H, f \in T x$ and $g \in T y$ imply $\langle x-y, f-g\rangle \geq 0$. A monotone mapping $T: H \rightarrow 2^{H}$ is maximal if its graph $G(T)$ is not properly contained in the graph of any other monotone mapping. It is known that a monotone mapping $T$ is maximal if and only if for $(x, f) \in H \times H,\langle x-y, f-g\rangle \geq 0$ for all $(y, g) \in G(T)$ implies $f \in T x$. Let $A: C \rightarrow H$ be a monotone, $L$-Lipschitz continuous mapping, and let $N_{C} v$ be the normal cone to $C$ at $v \in C$; that is, $N_{C^{v}}=\{w \in H$ : $\langle v-u, w\rangle \geq 0, \forall u \in C\}$. Define

$$
T v= \begin{cases}A v+N_{C} v, & \text { if } v \in C, \\ \emptyset, & \text { if } v \notin C .\end{cases}
$$

It is known that in this case $T$ is maximal monotone, and $0 \epsilon$ $T v$ if and only if $v \in \Omega$; see [40].

For solving the equilibrium problem, let us assume that the bifunction $F$ satisfies the following conditions:

(A1) $F(x, x)=0$ for all $x \in C$;

(A2) $F$ is monotone, that is, $F(x, y)+F(y, x) \leq 0$ for any $x, y \in C$;

(A3) $F$ is upper-hemicontinuous, that is, for each $x, y, z \in$ C,

$$
\limsup _{t \rightarrow 0^{+}} F(t z+(1-t) x, y) \leq F(x, y) ;
$$

(A4) $F(x, \cdot)$ is convex and lower semicontinuous for each $x \in C$;

(B1) for each $x \in H$ and $r>0$, there exists a bounded subset $D_{x} \subseteq C$ and $y_{x} \in C$ such that for any $z \in C \backslash D_{x}$,

$$
F\left(z, y_{x}\right)+\varphi\left(y_{x}\right)-\varphi(z)+\frac{1}{r}\left\langle y_{x}-z, z-x\right\rangle<0 ;
$$

(B2) $C$ is a bounded set.

Lemma 22 (see [41]). Assume that $F: C \times C \rightarrow \mathbf{R}$ satisfies (A1)-(A4) and let $\varphi: C \rightarrow \mathbf{R}$ be a proper lower semicontinuous and convex function. Assume that either (B1) or (B2) holds. For $r>0$ and $x \in H$, define a mapping $T_{r}^{(F, \varphi)}: H \rightarrow C$ as follows:

$$
\begin{aligned}
T_{r}^{(F, \varphi)}(x)=\{ & \{z \in C: F(z, y)+\varphi(y)-\varphi(z) \\
& \left.+\frac{1}{r}\langle y-z, z-x\rangle \geq 0, \forall y \in C\right\}
\end{aligned}
$$

for all $x \in H$. Then the following hold:

(1) for each $x \in H, T_{r}^{(F, \varphi)}(x) \neq \emptyset$;

(2) $T_{r}^{(F, \varphi)}$ is single-valued; 
(3) $T_{r}^{(F, \varphi)}$ is firmly nonexpansive; that is, for any $x, y \in H$,

$$
\left\|T_{r}^{(F, \varphi)} x-T_{r}^{(F, \varphi)} y\right\|^{2} \leq\left\langle T_{r}^{(F, \varphi)} x-T_{r}^{(F, \varphi)} y, x-y\right\rangle
$$

(4) $\operatorname{Fix}\left(T_{r}^{(F, \varphi)}\right)=\operatorname{MEP}(F, \varphi)$;

(5) $\operatorname{MEP}(F, \varphi)$ is closed and convex.

Lemma 23 (see [42]). Let $C$ be a closed convex subset of a real Hilbert space $H$. Let $\left\{x_{n}\right\}$ be a sequence in $H$ and $u \in H$. Let $q=P_{C} u$. If $\left\{x_{n}\right\}$ is such that $\omega_{w}\left(x_{n}\right) \subset C$ and satisfies the condition

$$
\left\|x_{n}-u\right\| \leq\|u-q\|, \quad \forall n,
$$

then $x_{n} \rightarrow$ qas $n \rightarrow \infty$.

\section{Strong Convergence Theorem}

In this section, we prove a strong convergence theorem for a hybrid extragradient iterative algorithm with regularization for finding a common element of the set of solutions of the CMP (2) for a convex functional $f: C \rightarrow \mathbf{R}$ with $L$ Lipschitz continuous gradient $\nabla f$, the set of solutions of finite generalized mixed equilibrium problems, the set of solutions of finite variational inequalities for inverse strong monotone mappings, and the set of fixed points of an asymptotically $\kappa$-strict pseudocontractive mapping $S: C \rightarrow C$ in the intermediate sense in a real Hilbert space. This iterative algorithm with regularization is based on the extragradient method, shrinking projection method, Mann-type iterative method, and hybrid gradient projection algorithm (GPA) with regularization.

Theorem 24. Let $C$ be a nonempty closed convex subset of a real Hilbert space $H$. Let $f: C \rightarrow \mathbf{R}$ be a convex functional with L-Lipschitz continuous gradient $\nabla f$. Let $M, N$ be two integers. Let $F_{k}$ be a bifunction from $C \times C$ to $\mathbf{R}$ satisfying (A1)-(A4) and let $\varphi_{k}: C \rightarrow \mathbf{R} \cup\{+\infty\}$ be a proper lower semicontinuous and convex function, where $k \in$ $\{1,2, \ldots, M\}$. Let $B_{k}$ and $A_{i}$ be $\mu_{k}$-inverse strongly monotone and $\eta_{i}$-inverse-strongly monotone, respectively, where $k \in$ $\{1,2, \ldots, M\}, i \in\{1,2, \ldots, N\}$. Let $S: C \rightarrow C$ be a uniformly continuous asymptotically $\kappa$-strict pseudocontractive mapping in the intermediate sense for some $0 \leq \kappa<1$ with sequence $\left\{\gamma_{n}\right\} \subset[0, \infty)$ such that $\lim _{n \rightarrow \infty} \gamma_{n}=0$ and $\left\{c_{n}\right\} \subset[0, \infty)$ such that $\lim _{n \rightarrow \infty} c_{n}=0$. Assume that $F:=\cap_{k=1}^{M} \operatorname{GMEP}\left(F_{k}, \varphi_{k}, A_{k}\right) \cap \cap_{i=1}^{N} \operatorname{VI}\left(C, A_{i}\right) \cap \operatorname{Fix}(S) \cap \Gamma$ is nonempty and bounded. Let $\left\{\alpha_{n}\right\}$ be a sequence in $[0, \infty)$ and let $\left\{\beta_{n}\right\},\left\{\delta_{n}\right\}$ be sequences in $[0,1]$ such that $0<a \leq \beta_{n} \leq 1$ and $\kappa \leq \delta_{n} \leq b<1$. Pick any $x_{0} \in H$ and set $C_{1}=C$, $x_{1}=P_{C_{1}} x_{0}$. Let $\left\{x_{n}\right\}$ be a sequence generated by the following algorithm:

$$
\begin{aligned}
u_{n}= & T_{r_{M, n}}^{\left(F_{M}, \varphi_{M}\right)}\left(I-r_{M, n} B_{M}\right) T_{r_{M, n}}^{\left(F_{M-1}, \varphi_{M-1}\right)} \\
\times & \left(I-r_{M-1, n} B_{M-1}\right) \cdots T_{r_{1, n}}^{\left(F_{1}, \varphi_{1}\right)}\left(I-r_{1, n} B_{1}\right) x_{n} \\
\tilde{x}_{n}= & P_{C}\left(I-\lambda_{N, n} A_{N}\right) P_{C}\left(I-\lambda_{N-1, n} A_{N-1}\right) \\
& \cdots P_{C}\left(I-\lambda_{2, n} A_{2}\right) P_{C}\left(I-\lambda_{1, n} A_{1}\right) u_{n},
\end{aligned}
$$

$$
\begin{gathered}
t_{n}=P_{C}\left(\tilde{x}_{n}-\lambda_{n} \nabla f_{\alpha_{n}}\left(\tilde{x}_{n}\right)\right), \\
z_{n}=P_{C}\left(\tilde{x}_{n}-\lambda_{n} \nabla f_{\alpha_{n}}\left(t_{n}\right)\right), \\
k_{n}=\delta_{n} z_{n}+\left(1-\delta_{n}\right) S^{n} z_{n}, \\
y_{n}=\left(1-\beta_{n}\right) x_{n}+\beta_{n} k_{n}, \\
C_{n+1}=\left\{z \in C_{n}:\left\|y_{n}-z\right\|^{2} \leq\left\|x_{n}-z\right\|^{2}+\theta_{n}\right\}, \\
x_{n+1}=P_{C_{n+1}} x_{0}, \quad \forall n \geq 0,
\end{gathered}
$$

where $\theta_{n}=\left(\gamma_{n}+\lambda_{n} \alpha_{n}\right) \Delta_{n}^{2}+3 \lambda_{n} \alpha_{n}\left(1+\gamma_{n}\right)^{2} \varrho^{2}+c_{n}, \Delta_{n}=$ $\sup \left\{\left\|x_{n}-p\right\|: p \in F\right\}<\infty$ and $=\sup \{\|p\|: p \in F\}<\infty$. Assume that the following conditions hold:

(i) $\lim _{n \rightarrow \infty} \alpha_{n}=0$;

(ii) $\left\{\lambda_{n}\right\} \subset[c, d]$ for some $c, d \in(0,(1 / L))$;

(iii) $\left\{\lambda_{i, n}\right\} \subset\left[a_{i}, b_{i}\right] \subset\left(0,2 \eta_{i}\right)$, for all $i \in\{1,2, \ldots, N\}$;

(iv) $\left\{r_{k, n}\right\} \subset\left[e_{k}, f_{k}\right] \subset\left(0,2 \mu_{k}\right)$, for all $k \in\{1,2, \ldots, M\}$.

Then $\left\{x_{n}\right\}$ converge strongly to $P_{F} x_{0}$ provided either (B1) or (B2) holds.

Proof. First of all, one can show that $P_{C}\left(I-\lambda \nabla f_{\alpha}\right)$ is $\xi$ averaged for each $\lambda \in(0,(2 /(\alpha+L)))$, where

$$
\xi=\frac{2+\lambda(\alpha+L)}{4} \in(0,1),
$$

which shows that $P_{C}\left(I-\lambda \nabla f_{\alpha}\right)$ is nonexpansive. Furthermore, for $\left\{\lambda_{n}\right\} \subset[c, d]$ with $c, d \in(0,(1 / L))$, we have

$$
c \leq \inf _{n \geq 1} \lambda_{n} \leq \sup _{n \geq 1} \lambda_{n} \leq d<\frac{1}{L}=\lim _{n \rightarrow \infty} \frac{1}{\alpha_{n}+L} .
$$

Without loss of generality, we may assume that

$$
c \leq \inf _{n \geq 1} \lambda_{n} \leq \sup _{n \geq 1} \lambda_{n} \leq d<\frac{1}{\alpha_{n}+L}, \quad \forall n \geq 1 .
$$

Consequently, it follows that for each integer $n \geq 1, P_{C}(I-$ $\left.\lambda_{n} \nabla f_{\alpha_{n}}\right)$ is $\xi_{n}$-averaged with

$$
\begin{aligned}
\xi_{n} & =\frac{1}{2}+\frac{\lambda_{n}\left(\alpha_{n}+L\right)}{2}-\frac{1}{2} \cdot \frac{\lambda_{n}\left(\alpha_{n}+L\right)}{2} \\
& =\frac{2+\lambda_{n}\left(\alpha_{n}+L\right)}{4} \in(0,1) .
\end{aligned}
$$

This immediately implies that $P_{C}\left(I-\lambda_{n} \nabla f_{\alpha_{n}}\right)$ is nonexpansive for all $n \geq 1$.

We divide the proof into several steps.

Step 1. We show that $\left\{x_{n}\right\}$ is well defined. It is obvious that $C_{n}$ is closed and convex. As the defining inequality in $C_{n}$ is equivalent to the inequality

$$
\left\langle 2\left(x_{n}-z_{n}\right), z\right\rangle \leq\left\|x_{n}\right\|^{2}-\left\|z_{n}\right\|^{2}+\theta_{n},
$$

by Lemma 13 we know that $C_{n}$ is convex for every $n \geq 1$. 
Next we show that $F \subset C_{n}$ for all $n \geq 1$. Put

$$
\begin{aligned}
\Theta_{n}^{k}= & T_{r_{k, n}}^{\left(F_{k}, \varphi_{k}\right)}\left(I-r_{k, n} B_{M}\right) T_{r_{k-1, n}}^{\left(F_{k-1}, \varphi_{k-1}\right)} \\
& \times\left(I-r_{k-1, n} B_{k-1}\right) \cdots T_{r_{1, n}}^{\left(F_{1}, \varphi_{1}\right)}\left(I-r_{1, n} B_{1}\right) x_{n}
\end{aligned}
$$

for all $k \in\{1,2, \ldots, M\}$ and $n \geq 1$,

$$
\begin{gathered}
\Omega_{n}^{i}=P_{C}\left(I-\lambda_{i, n} A_{i}\right) P_{C}\left(I-\lambda_{i-1, n} A_{i-1}\right) \\
\quad \cdots P_{C}\left(I-\lambda_{2, n} A_{2}\right) P_{C}\left(I-\lambda_{1, n} A_{1}\right)
\end{gathered}
$$

for all $i \in\{1,2, \ldots, N\}$ and $n \geq 1$, and $\Theta_{n}^{0}=\Omega_{n}^{0}=I$, where $I$ is the identity mapping on $H$. Then we have that $u_{n}=\Theta_{n}^{M} x_{n}$ and $\tilde{x}_{n}=\Omega_{n}^{N} u_{n}$. Suppose that $F \subset C_{n}$ for some $n \geq 1$. Take $p \in F$ arbitrarily. Then from (23) and Lemma 22 we have

$$
\begin{aligned}
& \left\|u_{n}-p\right\| \\
& =\| T_{r_{M, n}}^{\left(F_{M}, \varphi_{M}\right)}\left(I-r_{M, n} B_{M}\right) \Theta_{n}^{M-1} x_{n} \\
& \quad-T_{r_{M, n}}^{\left(F_{M}, \varphi_{M}\right)}\left(I-r_{M, n} B_{M}\right) \Theta_{n}^{M-1} p \| \\
& \leq\left\|\left(I-r_{M, n} B_{M}\right) \Theta_{n}^{M-1} x_{n}-\left(I-r_{M, n} B_{M}\right) \Theta_{n}^{M-1} p\right\| \\
& \leq\left\|\Theta_{n}^{M-1} x_{n}-\Theta_{n}^{M-1} p\right\| \\
& \quad \vdots \\
& \leq\left\|\Theta_{n}^{0} x_{n}-\Theta_{n}^{0} p\right\|=\left\|x_{n}-p\right\| .
\end{aligned}
$$

Similarly, we have

$$
\begin{aligned}
& \left\|\widetilde{x}_{n}-p\right\| \\
& =\left\|P_{C}\left(I-\lambda_{N, n} A_{N}\right) \Omega_{n}^{N-1} u_{n}-P_{C}\left(I-\lambda_{N, n} A_{N}\right) \Omega_{n}^{N-1} p\right\| \\
& \leq\left\|\left(I-\lambda_{N, n} A_{N}\right) \Omega_{n}^{N-1} u_{n}-\left(I-\lambda_{N, n} A_{N}\right) \Omega_{n}^{N-1} p\right\| \\
& \leq\left\|\Omega_{n}^{N-1} u_{n}-\Omega_{n}^{N-1} p\right\| \\
& \quad \vdots \\
& \leq\left\|\Omega_{n}^{0} x_{n}-\Omega_{n}^{0} p\right\|=\left\|u_{n}-p\right\| .
\end{aligned}
$$

Combining (52) and (53), we have

$$
\left\|\tilde{x}_{n}-p\right\| \leq\left\|x_{n}-p\right\|
$$

Also, it follows from (44) that

$$
\begin{aligned}
\| t_{n}- & p \| \\
= & \left\|P_{C}\left(I-\lambda_{n} \nabla f_{\alpha_{n}}\right) \tilde{x}_{n}-P_{C}\left(I-\lambda_{n} \nabla f\right) p\right\| \\
\leq & \left\|P_{C}\left(I-\lambda_{n} \nabla f_{\alpha_{n}}\right) \tilde{x}_{n}-P_{C}\left(I-\lambda_{n} \nabla f_{\alpha_{n}}\right) p\right\| \\
& +\left\|P_{C}\left(I-\lambda_{n} \nabla f_{\alpha_{n}}\right) p-P_{C}\left(I-\lambda_{n} \nabla f\right) p\right\| \\
\leq & \left\|\tilde{x}_{n}-p\right\|+\left\|\left(I-\lambda_{n} \nabla f_{\alpha_{n}}\right) p-\left(I-\lambda_{n} \nabla f\right) p\right\| \\
= & \left\|\tilde{x}_{n}-p\right\|+\lambda_{n} \alpha_{n}\|p\| .
\end{aligned}
$$

Note that $z_{n}=P_{C}\left(\tilde{x}_{n}-\lambda_{n} \nabla f_{\alpha_{n}}\left(t_{n}\right)\right)$ for every $n=1,2, \ldots$ Then, by Proposition 4(ii), we have

$$
\begin{aligned}
& \left\|z_{n}-p\right\|^{2} \\
& \leq\left\|\tilde{x}_{n}-\lambda_{n} \nabla f_{\alpha_{n}}\left(t_{n}\right)-p\right\|^{2}-\left\|\tilde{x}_{n}-\lambda_{n} \nabla f_{\alpha_{n}}\left(t_{n}\right)-z_{n}\right\|^{2} \\
& =\left\|\tilde{x}_{n}-p\right\|^{2}-\left\|\tilde{x}_{n}-z_{n}\right\|^{2}+2 \lambda_{n}\left\langle\nabla f_{\alpha_{n}}\left(t_{n}\right), p-z_{n}\right\rangle \\
& =\left\|\tilde{x}_{n}-p\right\|^{2}-\left\|\tilde{x}_{n}-z_{n}\right\|^{2} \\
& +2 \lambda_{n}\left(\left\langle\nabla f_{\alpha_{n}}\left(t_{n}\right)-\nabla f_{\alpha_{n}}(p), p-t_{n}\right\rangle\right. \\
& \left.+\left\langle\nabla f_{\alpha_{n}}(p), p-t_{n}\right\rangle+\left\langle\nabla f_{\alpha_{n}}\left(t_{n}\right), t_{n}-z_{n}\right\rangle\right) \\
& \leq\left\|\tilde{x}_{n}-p\right\|^{2}-\left\|\tilde{x}_{n}-z_{n}\right\|^{2} \\
& +2 \lambda_{n}\left(\left\langle\nabla f_{\alpha_{n}}(p), p-t_{n}\right\rangle+\left\langle\nabla f_{\alpha_{n}}\left(t_{n}\right), t_{n}-z_{n}\right\rangle\right) \\
& =\left\|\tilde{x}_{n}-p\right\|^{2}-\left\|\tilde{x}_{n}-z_{n}\right\|^{2} \\
& +2 \lambda_{n}\left[\left\langle\left(\alpha_{n} I+\nabla f\right) p, p-t_{n}\right\rangle+\left\langle\nabla f_{\alpha_{n}}\left(t_{n}\right), t_{n}-z_{n}\right\rangle\right] \\
& \leq\left\|\tilde{x}_{n}-p\right\|^{2}-\left\|\tilde{x}_{n}-z_{n}\right\|^{2} \\
& +2 \lambda_{n}\left[\alpha_{n}\left\langle p, p-t_{n}\right\rangle+\left\langle\nabla f_{\alpha_{n}}\left(t_{n}\right), t_{n}-z_{n}\right\rangle\right] \\
& =\left\|\tilde{x}_{n}-p\right\|^{2}-\left\|\tilde{x}_{n}-t_{n}\right\|^{2} \\
& -2\left\langle\tilde{x}_{n}-t_{n}, t_{n}-z_{n}\right\rangle-\left\|t_{n}-z_{n}\right\|^{2} \\
& +2 \lambda_{n}\left[\alpha_{n}\left\langle p, p-t_{n}\right\rangle+\left\langle\nabla f_{\alpha_{n}}\left(t_{n}\right), t_{n}-z_{n}\right\rangle\right] \\
& =\left\|\tilde{x}_{n}-p\right\|^{2}-\left\|\tilde{x}_{n}-t_{n}\right\|^{2}-\left\|t_{n}-z_{n}\right\|^{2} \\
& +2\left\langle\tilde{x}_{n}-\lambda_{n} \nabla f_{\alpha_{n}}\left(t_{n}\right)-t_{n}, z_{n}-t_{n}\right\rangle \\
& +2 \lambda_{n} \alpha_{n}\left\langle p, p-t_{n}\right\rangle \text {. }
\end{aligned}
$$


Further, by Proposition 4(i), we have

$$
\begin{aligned}
\left\langle\widetilde{x}_{n}\right. & \left.-\lambda_{n} \nabla f_{\alpha_{n}}\left(t_{n}\right)-t_{n}, z_{n}-t_{n}\right\rangle \\
= & \left\langle\widetilde{x}_{n}-\lambda_{n} \nabla f_{\alpha_{n}}\left(\widetilde{x}_{n}\right)-t_{n}, z_{n}-t_{n}\right\rangle \\
& +\left\langle\lambda_{n} \nabla f_{\alpha_{n}}\left(\widetilde{x}_{n}\right)-\lambda_{n} \nabla f_{\alpha_{n}}\left(t_{n}\right), z_{n}-t_{n}\right\rangle \\
\leq & \left\langle\lambda_{n} \nabla f_{\alpha_{n}}\left(\widetilde{x}_{n}\right)-\lambda_{n} \nabla f_{\alpha_{n}}\left(t_{n}\right), z_{n}-t_{n}\right\rangle \\
\leq & \lambda_{n}\left\|\nabla f_{\alpha_{n}}\left(\widetilde{x}_{n}\right)-\nabla f_{\alpha_{n}}\left(t_{n}\right)\right\|\left\|z_{n}-t_{n}\right\| \\
\leq & \lambda_{n}\left(\alpha_{n}+L\right)\left\|\widetilde{x}_{n}-t_{n}\right\|\left\|z_{n}-t_{n}\right\| .
\end{aligned}
$$

So from (54) and (55), we obtain

$$
\begin{aligned}
\left\|z_{n}-p\right\|^{2} \leq & \left\|\tilde{x}_{n}-p\right\|^{2}-\left\|\tilde{x}_{n}-t_{n}\right\|^{2}-\left\|t_{n}-z_{n}\right\|^{2} \\
& +2 \lambda_{n}\left(\alpha_{n}+L\right)\left\|\tilde{x}_{n}-t_{n}\right\|\left\|z_{n}-t_{n}\right\| \\
& +2 \lambda_{n} \alpha_{n}\left\langle p, p-t_{n}\right\rangle \\
\leq & \left\|\widetilde{x}_{n}-p\right\|^{2}-\left\|\widetilde{x}_{n}-t_{n}\right\|^{2}-\left\|t_{n}-z_{n}\right\|^{2} \\
& +\lambda_{n}^{2}\left(\alpha_{n}+L\right)^{2}\left\|\tilde{x}_{n}-t_{n}\right\|^{2}+\left\|t_{n}-z_{n}\right\|^{2} \\
& +2 \lambda_{n} \alpha_{n}\|p\|\left\|p-t_{n}\right\| \\
= & \left\|\tilde{x}_{n}-p\right\|^{2}+2 \lambda_{n} \alpha_{n}\|p\|\left\|p-t_{n}\right\| \\
& +\left(\lambda_{n}^{2}\left(\alpha_{n}+L\right)^{2}-1\right)\left\|\tilde{x}_{n}-t_{n}\right\|^{2} \\
\leq & \left\|\widetilde{x}_{n}-p\right\|^{2}+2 \lambda_{n} \alpha_{n}\|p\|\left\|p-t_{n}\right\| \\
\leq & \left\|\widetilde{x}_{n}-p\right\|^{2}+2 \lambda_{n} \alpha_{n}\|p\|\left[\left\|\tilde{x}_{n}-p\right\|+\lambda_{n} \alpha_{n}\|p\|\right] \\
\leq & \left\|x_{n}-p\right\|^{2}+2 \lambda_{n} \alpha_{n}\|p\|\left[\left\|x_{n}-p\right\|+\lambda_{n} \alpha_{n}\|p\|\right] \\
= & \left\|x_{n}-p\right\|^{2}+2 \lambda_{n} \alpha_{n}\|p\|\left\|x_{n}-p\right\|+2 \lambda_{n}^{2} \alpha_{n}^{2}\|p\|^{2} .
\end{aligned}
$$

By Lemma 12 and (58), we have

$$
\begin{aligned}
\| k_{n}- & p \|^{2} \\
= & \left\|\delta_{n}\left(z_{n}-p\right)+\left(1-\delta_{n}\right)\left(S^{n} z_{n}-p\right)\right\|^{2} \\
= & \delta_{n}\left\|z_{n}-p\right\|^{2}+\left(1-\delta_{n}\right)\left\|S^{n} z_{n}-p\right\|^{2} \\
& -\delta_{n}\left(1-\delta_{n}\right)\left\|z_{n}-S^{n} z_{n}\right\|^{2} \\
\leq & \delta_{n}\left\|z_{n}-p\right\|^{2}+\left(1-\delta_{n}\right) \\
& \times\left[\left(1+\gamma_{n}\right)\left\|z_{n}-p\right\|^{2}+\kappa\left\|z_{n}-S^{n} z_{n}\right\|^{2}+c_{n}\right] \\
& -\delta_{n}\left(1-\delta_{n}\right)\left\|z_{n}-S^{n} z_{n}\right\|^{2}
\end{aligned}
$$

$$
\begin{aligned}
&= {\left[1+\gamma_{n}\left(1-\delta_{n}\right)\right]\left\|z_{n}-p\right\|^{2} } \\
&+\left(1-\delta_{n}\right)\left(\kappa-\delta_{n}\right)\left\|z_{n}-S^{n} z_{n}\right\|^{2}+\left(1-\delta_{n}\right) c_{n} \\
& \leq {\left[1+\gamma_{n}\left(1-\delta_{n}\right)\right] } \\
& \times\left[\left\|x_{n}-p\right\|^{2}+2 \lambda_{n} \alpha_{n}\|p\|\left\|x_{n}-p\right\|+2 \lambda_{n}^{2} \alpha_{n}^{2}\|p\|^{2}\right] \\
&+\left(1-\delta_{n}\right)\left(\kappa-\delta_{n}\right)\left\|z_{n}-S^{n} z_{n}\right\|^{2}+c_{n} \\
& \leq {\left[1+\gamma_{n}\left(1-\delta_{n}\right)\right]\left[\left\|x_{n}-p\right\|^{2}+2 \lambda_{n} \alpha_{n}\|p\|\right.} \\
&\left.\quad \times\left\|x_{n}-p\right\|+2 \lambda_{n}^{2} \alpha_{n}^{2}\|p\|^{2}\right]+c_{n} \\
& \leq
\end{aligned}
$$

It follows from (59) and $\lambda_{n}\left(\alpha_{n}+L\right)<1$ that

$$
\begin{aligned}
\| y_{n}- & p \|^{2} \\
= & \left\|\left(1-\beta_{n}\right)\left(x_{n}-p\right)+\beta_{n}\left(k_{n}-p\right)\right\|^{2} \\
\leq & \left(1-\beta_{n}\right)\left\|x_{n}-p\right\|^{2}+\beta_{n}\left\|k_{n}-p\right\|^{2} \\
\leq & \left(1-\beta_{n}\right)\left\|x_{n}-p\right\|^{2} \\
& +\beta_{n}\left\{( 1 + \gamma _ { n } ) \left[\left\|x_{n}-p\right\|^{2}+2 \lambda_{n} \alpha_{n}\|p\|\left\|x_{n}-p\right\|\right.\right. \\
& \left.\left.\quad+2 \lambda_{n}^{2} \alpha_{n}^{2}\|p\|^{2}\right]+c_{n}\right\} \\
\leq & \left(1+\gamma_{n}\right)\left[\left\|x_{n}-p\right\|^{2}+2 \lambda_{n} \alpha_{n}\|p\|\left\|x_{n}-p\right\|\right. \\
& \left.+2 \lambda_{n}^{2} \alpha_{n}^{2}\|p\|^{2}\right]+c_{n} \\
= & \left(1+\gamma_{n}\right)\left\|x_{n}-p\right\|^{2}+2 \lambda_{n} \alpha_{n}\left(1+\gamma_{n}\right)\|p\|\left\|x_{n}-p\right\| \\
& +2\left(1+\gamma_{n}\right) \lambda_{n}^{2} \alpha_{n}^{2}\|p\|^{2}+c_{n} \\
\leq & \left(1+\gamma_{n}\right)\left\|x_{n}-p\right\|^{2} \\
& +\lambda_{n} \alpha_{n}\left[\left(1+\gamma_{n}\right)^{2}\|p\|^{2}+\left\|x_{n}-p\right\|^{2}\right] \\
& +2\left(1+\gamma_{n}\right) \lambda_{n}^{2} \alpha_{n}^{2}\|p\|^{2}+c_{n} \\
\leq & \left(1+\gamma_{n}\right)\left\|x_{n}-p\right\|^{2} \\
& +\lambda_{n} \alpha_{n}\left[\left(1+\gamma_{n}\right)^{2}\|p\|^{2}+\left\|x_{n}-p\right\|^{2}\right] \\
& +2\left(1+\gamma_{n}\right)^{2} \lambda_{n} \alpha_{n}\|p\|^{2}+c_{n}
\end{aligned}
$$




$$
\begin{aligned}
= & \left(1+\gamma_{n}\right)\left\|x_{n}-p\right\|^{2} \\
& +\lambda_{n} \alpha_{n}\left[3\left(1+\gamma_{n}\right)^{2}\|p\|^{2}+\left\|x_{n}-p\right\|^{2}\right]+c_{n} \\
\leq & \left\|x_{n}-p\right\|^{2}+\left(\gamma_{n}+\lambda_{n} \alpha_{n}\right) \Delta_{n}^{2} \\
& +3 \lambda_{n} \alpha_{n}\left(1+\gamma_{n}\right)^{2} \varrho^{2}+c_{n}=\left\|x_{n}-p\right\|^{2}+\theta_{n} .
\end{aligned}
$$

Hence $p \in C_{n+1}$. This implies that $F \subset C_{n}$ for all $n \geq 1$. Therefore, $\left\{x_{n}\right\}$ is well defined.

Step 2. We prove that $\left\|x_{n}-k_{n}\right\| \rightarrow 0$ as $n \rightarrow \infty$.

Indeed, let $v=P_{F} x_{0}$. From $x_{n}=P_{C_{n}} x_{0}$ and $v \in F \subset C_{n}$, we obtain

$$
\left\|x_{n}-x_{0}\right\| \leq\left\|v-x_{0}\right\|
$$

This implies that $\left\{x_{n}\right\}$ is bounded and hence $\left\{u_{n}\right\}$, $\left\{\tilde{x}_{n}\right\},\left\{t_{n}\right\},\left\{z_{n}\right\},\left\{k_{n}\right\}$, and $\left\{y_{n}\right\}$ are also bounded. Since $x_{n+1} \in C_{n+1} \subset C_{n}$ and $x_{n}=P_{C_{n}} x_{0}$, we have

$$
\left\|x_{n}-x_{0}\right\| \leq\left\|x_{n+1}-x_{0}\right\|, \quad \forall n \geq 1
$$

Therefore, $\lim _{n \rightarrow \infty}\left\|x_{n}-x_{0}\right\|$ exists. From $x_{n}=P_{C_{n}} x_{0}, x_{n+1} \in$ $C_{n+1} \subset C_{n}$, we obtain

$$
\left\|x_{n+1}-x_{n}\right\|^{2} \leq\left\|x_{0}-x_{n+1}\right\|^{2}-\left\|x_{0}-x_{n}\right\|^{2}
$$

which implies

$$
\lim _{n \rightarrow \infty}\left\|x_{n+1}-x_{n}\right\|=0
$$

It follows from $x_{n+1} \in C_{n+1}$ that $\left\|y_{n}-x_{n+1}\right\|^{2} \leq\left\|x_{n}-x_{n+1}\right\|^{2}+$ $\theta_{n}$ and hence

$$
\begin{aligned}
\left\|x_{n}-y_{n}\right\|^{2} & \leq 2\left(\left\|x_{n}-x_{n+1}\right\|^{2}+\left\|x_{n+1}-y_{n}\right\|^{2}\right) \\
& \leq 2\left(\left\|x_{n}-x_{n+1}\right\|^{2}+\left\|x_{n}-x_{n+1}\right\|^{2}+\theta_{n}\right) \\
& =2\left(2\left\|x_{n}-x_{n+1}\right\|^{2}+\theta_{n}\right) .
\end{aligned}
$$

From (64) and $\lim _{n \rightarrow \infty} \theta_{n}=0$, we have

$$
\lim _{n \rightarrow \infty}\left\|x_{n}-y_{n}\right\|=0
$$

Note that

$$
y_{n}-x_{n}=\beta_{n}\left(k_{n}-x_{n}\right)
$$

Since $0<a \leq \beta_{n}$ and (66), we obtain

$$
\lim _{n \rightarrow \infty}\left\|x_{n}-k_{n}\right\|=0
$$

Step 3. We prove that $\left\|x_{n}-u_{n}\right\| \rightarrow 0,\left\|u_{n}-z_{n}\right\| \rightarrow 0$, and $\left\|z_{n}-S^{n} z_{n}\right\| \rightarrow 0$ as $n \rightarrow \infty$.
Indeed, from (58) and (59) it follows that

$$
\begin{aligned}
\left\|k_{n}-p\right\|^{2} \leq & {\left[1+\gamma_{n}\left(1-\delta_{n}\right)\right]\left\|z_{n}-p\right\|^{2} } \\
& +\left(1-\delta_{n}\right)\left(\kappa-\delta_{n}\right)\left\|z_{n}-S^{n} z_{n}\right\|^{2} \\
& +\left(1-\delta_{n}\right) c_{n} \\
\leq & \left\|z_{n}-p\right\|^{2}+\gamma_{n}\left\|z_{n}-p\right\|^{2}+c_{n} \\
\leq & \left\|z_{n}-p\right\|^{2} \\
& +\gamma_{n}\left[\left\|x_{n}-p\right\|^{2}+2 \lambda_{n} \alpha_{n}\|p\|\left\|x_{n}-p\right\|\right. \\
& \left.+2 \lambda_{n}^{2} \alpha_{n}^{2}\|p\|^{2}\right]+c_{n} \\
\leq & \left\|z_{n}-p\right\|^{2} \\
& +\gamma_{n}\left[\left\|x_{n}-p\right\|^{2}+2 \sqrt{2} \lambda_{n} \alpha_{n}\|p\|\left\|x_{n}-p\right\|\right. \\
& \left.+2 \lambda_{n}^{2} \alpha_{n}^{2}\|p\|^{2}\right]+c_{n} \\
& +\gamma_{n}\left(\left\|x_{n}-p\right\|+\sqrt{2} \lambda_{n} \alpha_{n}\|p\|\right)^{2}+c_{n} . \\
& \quad p \|^{2}
\end{aligned}
$$

Next we prove that

$$
\lim _{n \rightarrow \infty}\left\|\Theta_{n}^{k} x_{n}-\Theta_{n}^{k-1} x_{n}\right\|=0, \quad k=1,2, \ldots, M
$$

For $p \in F$, it follows from (23) that

$$
\begin{aligned}
\left\|\Theta_{n}^{k} x_{n}-p\right\|^{2}= & \| T_{r_{k, n}}^{\left(F_{k}, \varphi_{k}\right)}\left(I-r_{k, n} B_{k}\right) \Theta_{n}^{k-1} x_{n} \\
& -T_{r_{k, n}}^{\left(F_{k}, \varphi_{k}\right)}\left(I-r_{k, n} B_{k}\right) p \|^{2} \\
\leq & \left\|\left(I-r_{k, n} B_{k}\right) \Theta_{n}^{k-1} x_{n}-\left(I-r_{k, n} B_{k}\right) p\right\|^{2} \\
\leq & \left\|\Theta_{n}^{k-1} x_{n}-p\right\|^{2}+r_{k, n}\left(r_{k, n}-2 \mu_{k}\right) \\
& \times\left\|B_{k} \Theta_{n}^{k-1} x_{n}-B_{k} p\right\|^{2} \\
\leq & \left\|x_{n}-p\right\|^{2}+r_{k, n}\left(r_{k, n}-2 \mu_{k}\right) \\
& \times\left\|B_{k} \Theta_{n}^{k-1} x_{n}-B_{k} p\right\|^{2} .
\end{aligned}
$$


By (52), (53), (58), (69), and (71), we obtain

$$
\begin{aligned}
\| k_{n}- & p \|^{2} \\
\leq & \left\|z_{n}-p\right\|^{2}+\gamma_{n}\left(\left\|x_{n}-p\right\|+\sqrt{2} \lambda_{n} \alpha_{n}\|p\|\right)^{2}+c_{n} \\
\leq & \left\|\tilde{x}_{n}-p\right\|^{2}+2 \lambda_{n} \alpha_{n}\|p\|\left[\left\|\tilde{x}_{n}-p\right\|+\lambda_{n} \alpha_{n}\|p\|\right] \\
& +\gamma_{n}\left(\left\|x_{n}-p\right\|+\sqrt{2} \lambda_{n} \alpha_{n}\|p\|\right)^{2}+c_{n} \\
\leq & \left\|u_{n}-p\right\|^{2}+2 \lambda_{n} \alpha_{n}\|p\|\left[\left\|u_{n}-p\right\|+\lambda_{n} \alpha_{n}\|p\|\right] \\
& +\gamma_{n}\left(\left\|x_{n}-p\right\|+\sqrt{2} \lambda_{n} \alpha_{n}\|p\|\right)^{2}+c_{n} \\
\leq & \left\|u_{n}-p\right\|^{2}+2 \lambda_{n} \alpha_{n}\|p\|\left[\left\|x_{n}-p\right\|+\lambda_{n} \alpha_{n}\|p\|\right] \\
& +\gamma_{n}\left(\left\|x_{n}-p\right\|+\sqrt{2} \lambda_{n} \alpha_{n}\|p\|\right)^{2}+c_{n} \\
\leq & \left\|\Theta_{n}^{k} x_{n}-p\right\|^{2}+2 \lambda_{n} \alpha_{n}\|p\|\left[\left\|x_{n}-p\right\|+\lambda_{n} \alpha_{n}\|p\|\right] \\
& +\gamma_{n}\left(\left\|x_{n}-p\right\|+\sqrt{2} \lambda_{n} \alpha_{n}\|p\|\right)^{2}+c_{n} \\
\leq & \left\|x_{n}-p\right\|^{2}+r_{k, n}\left(r_{k, n}-2 \mu_{k}\right)\left\|B_{k} \Theta_{n}^{k-1} x_{n}-B_{k} p\right\|^{2} \\
& +2 \lambda_{n} \alpha_{n}\|p\|\left[\left\|x_{n}-p\right\|+\lambda_{n} \alpha_{n}\|p\|\right] \\
& +\gamma_{n}\left(\left\|x_{n}-p\right\|+\sqrt{2} \lambda_{n} \alpha_{n}\|p\|\right)^{2}+c_{n},
\end{aligned}
$$

which implies that

$$
\begin{aligned}
& r_{k, n}\left(2 \mu_{k}-r_{k, n}\right)\left\|B_{k} \Theta_{n}^{k-1} x_{n}-B_{k} p\right\|^{2} \\
& \leq\left\|x_{n}-p\right\|^{2}-\left\|k_{n}-p\right\|^{2} \\
&+2 \lambda_{n} \alpha_{n}\|p\|\left[\left\|x_{n}-p\right\|+\lambda_{n} \alpha_{n}\|p\|\right] \\
&+\gamma_{n}\left(\left\|x_{n}-p\right\|+\sqrt{2} \lambda_{n} \alpha_{n}\|p\|\right)^{2}+c_{n} \\
& \leq\left\|x_{n}-k_{n}\right\|\left(\left\|x_{n}-p\right\|+\left\|k_{n}-p\right\|\right) \\
&+2 \lambda_{n} \alpha_{n}\|p\|\left[\left\|x_{n}-p\right\|+\lambda_{n} \alpha_{n}\|p\|\right] \\
&+\gamma_{n}\left(\left\|x_{n}-p\right\|+\sqrt{2} \lambda_{n} \alpha_{n}\|p\|\right)^{2}+c_{n} .
\end{aligned}
$$

Since $\left\{\lambda_{n}\right\} \subset[c, d] \subset(0,1 / L),\left\{r_{k, n}\right\} \subset\left[e_{k}, f_{k}\right] \subset\left(0,2 \mu_{k}\right), k \in$ $\{1,2, \ldots, M\}, \alpha_{n} \rightarrow 0, \gamma_{n} \rightarrow 0$, and $c_{n} \rightarrow 0$, we conclude from (68) and the boundedness of $\left\{x_{n}\right\},\left\{k_{n}\right\}$ that

$$
\lim _{n \rightarrow \infty}\left\|B_{k} \Theta_{n}^{k-1} x_{n}-B_{k} p\right\|=0, \quad k=1,2, \ldots, M .
$$

By Lemmas 12 and 22, we have

$$
\begin{gathered}
\left\|\Theta_{n}^{k} x_{n}-p\right\|^{2} \\
=\left\|T_{r_{k, n}}^{\left(F_{k}, \varphi_{k}\right)}\left(I-r_{k, n} B_{k}\right) \Theta_{n}^{k-1} x_{n}-T_{r_{k, n}}^{\left(F_{k}, \varphi_{k}\right)}\left(I-r_{k, n} B_{k}\right) p\right\|^{2} \\
\leq\left\langle\left(I-r_{k, n} B_{k}\right) \Theta_{n}^{k-1} x_{n}-\left(I-r_{k, n} B_{k}\right) p, \Theta_{n}^{k} x_{n}-p\right\rangle \\
=\frac{1}{2}\left(\left\|\left(I-r_{k, n} B_{k}\right) \Theta_{n}^{k-1} x_{n}-\left(I-r_{k, n} B_{k}\right) p\right\|^{2}\right. \\
\quad+\left\|\Theta_{n}^{k} x_{n}-p\right\|^{2}-\|\left(I-r_{k, n} B_{k}\right) \Theta_{n}^{k-1} x_{n} \\
\left.\quad-\left(I-r_{k, n} B_{k}\right) p-\left(\Theta_{n}^{k} x_{n}-p\right) \|^{2}\right) \\
\leq \frac{1}{2}\left(\left\|\Theta_{n}^{k-1} x_{n}-p\right\|^{2}+\left\|\Theta_{n}^{k} x_{n}-p\right\|^{2}\right. \\
\left.\quad-\left\|\Theta_{n}^{k-1} x_{n}-\Theta_{n}^{k} x_{n}-r_{k, n}\left(B_{k} \Theta_{n}^{k-1} x_{n}-B_{k} p\right)\right\|^{2}\right),
\end{gathered}
$$

which implies that

$$
\begin{aligned}
\left\|\Theta_{n}^{k} x_{n}-p\right\|^{2} \leq & \left\|\Theta_{n}^{k-1} x_{n}-p\right\|^{2} \\
- & \| \Theta_{n}^{k-1} x_{n}-\Theta_{n}^{k} x_{n} \\
& -r_{k, n}\left(B_{k} \Theta_{n}^{k-1} x_{n}-B_{k} p\right) \|^{2} \\
= & \left\|\Theta_{n}^{k-1} x_{n}-p\right\|^{2}-\left\|\Theta_{n}^{k-1} x_{n}-\Theta_{n}^{k} x_{n}\right\|^{2} \\
& -r_{k, n}^{2}\left\|B_{k} \Theta_{n}^{k-1} x_{n}-B_{k} p\right\|^{2} \\
& +2 r_{k, n}\left\langle\Theta_{n}^{k-1} x_{n}-\Theta_{n}^{k} x_{n}, B_{k} \Theta_{n}^{k-1} x_{n}-B_{k} p\right\rangle \\
\leq & \left\|\Theta_{n}^{k-1} x_{n}-p\right\|^{2}-\left\|\Theta_{n}^{k-1} x_{n}-\Theta_{n}^{k} x_{n}\right\|^{2} \\
& +2 r_{k, n}\left\|\Theta_{n}^{k-1} x_{n}-\Theta_{n}^{k} x_{n}\right\|\left\|B_{k} \Theta_{n}^{k-1} x_{n}-B_{k} p\right\| \\
\leq & \left\|x_{n}-p\right\|^{2}-\left\|\Theta_{n}^{k-1} x_{n}-\Theta_{n}^{k} x_{n}\right\|^{2} \\
& +2 r_{k, n}\left\|\Theta_{n}^{k-1} x_{n}-\Theta_{n}^{k} x_{n}\right\|\left\|B_{k} \Theta_{n}^{k-1} x_{n}-B_{k} p\right\| .
\end{aligned}
$$

Utilizing (72) and (76), we have

$$
\begin{aligned}
& \left\|k_{n}-p\right\|^{2} \\
& \leq\left\|\Theta_{n}^{k} x_{n}-p\right\|^{2}+2 \lambda_{n} \alpha_{n}\|p\|\left[\left\|x_{n}-p\right\|+\lambda_{n} \alpha_{n}\|p\|\right] \\
& \quad+\gamma_{n}\left(\left\|x_{n}-p\right\|+\sqrt{2} \lambda_{n} \alpha_{n}\|p\|\right)^{2}+c_{n}
\end{aligned}
$$




$$
\begin{aligned}
\leq & \left\|x_{n}-p\right\|^{2}-\left\|\Theta_{n}^{k-1} x_{n}-\Theta_{n}^{k} x_{n}\right\|^{2} \\
& +2 r_{k, n}\left\|\Theta_{n}^{k-1} x_{n}-\Theta_{n}^{k} x_{n}\right\|\left\|B_{k} \Theta_{n}^{k-1} x_{n}-B_{k} p\right\| \\
& +2 \lambda_{n} \alpha_{n}\|p\|\left[\left\|x_{n}-p\right\|+\lambda_{n} \alpha_{n}\|p\|\right] \\
& +\gamma_{n}\left(\left\|x_{n}-p\right\|+\sqrt{2} \lambda_{n} \alpha_{n}\|p\|\right)^{2}+c_{n},
\end{aligned}
$$

which implies

$$
\begin{aligned}
& \left\|\Theta_{n}^{k-1} x_{n}-\Theta_{n}^{k} x_{n}\right\|^{2} \\
& \leq\left\|x_{n}-p\right\|^{2}-\left\|k_{n}-p\right\|^{2} \\
& \quad+2 r_{k, n}\left\|\Theta_{n}^{k-1} x_{n}-\Theta_{n}^{k} x_{n}\right\|\left\|B_{k} \Theta_{n}^{k-1} x_{n}-B_{k} p\right\| \\
& \quad+2 \lambda_{n} \alpha_{n}\|p\|\left[\left\|x_{n}-p\right\|+\lambda_{n} \alpha_{n}\|p\|\right] \\
& \quad+\gamma_{n}\left(\left\|x_{n}-p\right\|+\sqrt{2} \lambda_{n} \alpha_{n}\|p\|\right)^{2}+c_{n} \\
& \leq\left\|x_{n}-k_{n}\right\|\left(\left\|x_{n}-p\right\|+\left\|k_{n}-p\right\|\right) \\
& \quad+2 r_{k, n}\left\|\Theta_{n}^{k-1} x_{n}-\Theta_{n}^{k} x_{n}\right\|\left\|B_{k} \Theta_{n}^{k-1} x_{n}-B_{k} p\right\| \\
& \quad+2 \lambda_{n} \alpha_{n}\|p\|\left[\left\|x_{n}-p\right\|+\lambda_{n} \alpha_{n}\|p\|\right] \\
& \quad+\gamma_{n}\left(\left\|x_{n}-p\right\|+\sqrt{2} \lambda_{n} \alpha_{n}\|p\|\right)^{2}+c_{n} .
\end{aligned}
$$

Since $\left\{\lambda_{n}\right\} \subset[c, d] \subset(0,1 / L),\left\{r_{k, n}\right\} \subset\left[e_{k}, f_{k}\right] \subset\left(0,2 \mu_{k}\right), k \in$ $\{1,2, \ldots, M\}, \alpha_{n} \rightarrow 0, \gamma_{n} \rightarrow 0$, and $c_{n} \rightarrow 0$, we conclude from (68) and (74) and the boundedness of $\left\{x_{n}\right\},\left\{k_{n}\right\}$ that (70) holds. Hence we obtain

$$
\begin{aligned}
\left\|x_{n}-u_{n}\right\|= & \left\|\Theta_{n}^{0} x_{n}-\Theta_{n}^{M} x_{n}\right\| \\
\leq & \left\|\Theta_{n}^{0} x_{n}-\Theta_{n}^{1} x_{n}\right\|+\left\|\Theta_{n}^{1} x_{n}-\Theta_{n}^{2} x_{n}\right\| \\
& +\cdots+\left\|\Theta_{n}^{M-1} x_{n}-\Theta_{n}^{M} x_{n}\right\| \\
& \longrightarrow 0 \text { as } n \longrightarrow \infty .
\end{aligned}
$$

Next we show that $\lim _{n \rightarrow \infty}\left\|A_{i} \Omega_{n}^{i} u_{n}-A_{i} p\right\|=0, i=$ $1,2, \ldots, N$. It follows from $(23)$ that

$$
\begin{aligned}
& \left\|\Omega_{n}^{i} u_{n}-p\right\|^{2} \\
& \quad=\left\|P_{C}\left(I-\lambda_{i, n} A_{i}\right) \Omega_{n}^{i-1} u_{n}-P_{C}\left(I-\lambda_{i, n} A_{i}\right) p\right\|^{2} \\
& \quad \leq\left\|\left(I-\lambda_{i, n} A_{i}\right) \Omega_{n}^{i-1} u_{n}-\left(I-\lambda_{i, n} A_{i}\right) p\right\|^{2} \\
& \quad \leq\left\|\Omega_{n}^{i-1} u_{n}-p\right\|^{2}+\lambda_{i, n}\left(\lambda_{i, n}-2 \eta_{i}\right)\left\|A_{i} \Omega_{n}^{i-1} u_{n}-A_{i} p\right\|^{2} \\
& \quad \leq\left\|u_{n}-p\right\|^{2}+\lambda_{i, n}\left(\lambda_{i, n}-2 \eta_{i}\right)\left\|A_{i} \Omega_{n}^{i-1} u_{n}-A_{i} p\right\|^{2} \\
& \quad \leq\left\|x_{n}-p\right\|^{2}+\lambda_{i, n}\left(\lambda_{i, n}-2 \eta_{i}\right)\left\|A_{i} \Omega_{n}^{i-1} u_{n}-A_{i} p\right\|^{2} .
\end{aligned}
$$

Utilizing (54), (72), and (80), we get

$$
\begin{aligned}
\| k_{n}- & p \|^{2} \\
\leq & \left\|\tilde{x}_{n}-p\right\|^{2}+2 \lambda_{n} \alpha_{n}\|p\|\left[\left\|\tilde{x}_{n}-p\right\|+\lambda_{n} \alpha_{n}\|p\|\right] \\
& +\gamma_{n}\left(\left\|x_{n}-p\right\|+\sqrt{2} \lambda_{n} \alpha_{n}\|p\|\right)^{2}+c_{n} \\
\leq & \left\|\tilde{x}_{n}-p\right\|^{2}+2 \lambda_{n} \alpha_{n}\|p\|\left[\left\|x_{n}-p\right\|+\lambda_{n} \alpha_{n}\|p\|\right] \\
& +\gamma_{n}\left(\left\|x_{n}-p\right\|+\sqrt{2} \lambda_{n} \alpha_{n}\|p\|\right)^{2}+c_{n} \\
\leq & \left\|\Omega_{n}^{i} u_{n}-p\right\|^{2}+2 \lambda_{n} \alpha_{n}\|p\|\left[\left\|x_{n}-p\right\|+\lambda_{n} \alpha_{n}\|p\|\right] \\
& +\gamma_{n}\left(\left\|x_{n}-p\right\|+\sqrt{2} \lambda_{n} \alpha_{n}\|p\|\right)^{2}+c_{n} \\
\leq & \left\|x_{n}-p\right\|^{2}+\lambda_{i, n}\left(\lambda_{i, n}-2 \eta_{i}\right)\left\|A_{i} \Omega_{n}^{i-1} u_{n}-A_{i} p\right\|^{2} \\
& +2 \lambda_{n} \alpha_{n}\|p\|\left[\left\|x_{n}-p\right\|+\lambda_{n} \alpha_{n}\|p\|\right] \\
& +\gamma_{n}\left(\left\|x_{n}-p\right\|+\sqrt{2} \lambda_{n} \alpha_{n}\|p\|\right)^{2}+c_{n},
\end{aligned}
$$

which implies

$$
\begin{aligned}
\lambda_{i, n}\left(2 \eta_{i}-\lambda_{i, n}\right)\left\|A_{i} \Omega_{n}^{i-1} u_{n}-A_{i} p\right\|^{2} \\
\leq\left\|x_{n}-p\right\|^{2}-\left\|k_{n}-p\right\|^{2} \\
\quad+2 \lambda_{n} \alpha_{n}\|p\|\left[\left\|x_{n}-p\right\|+\lambda_{n} \alpha_{n}\|p\|\right] \\
\quad+\gamma_{n}\left(\left\|x_{n}-p\right\|+\sqrt{2} \lambda_{n} \alpha_{n}\|p\|\right)^{2}+c_{n} \\
\leq\left\|x_{n}-k_{n}\right\|\left(\left\|x_{n}-p\right\|+\left\|k_{n}-p\right\|\right) \\
\quad+2 \lambda_{n} \alpha_{n}\|p\|\left[\left\|x_{n}-p\right\|+\lambda_{n} \alpha_{n}\|p\|\right] \\
\quad+\gamma_{n}\left(\left\|x_{n}-p\right\|+\sqrt{2} \lambda_{n} \alpha_{n}\|p\|\right)^{2}+c_{n} .
\end{aligned}
$$

Since $\left\{\lambda_{n}\right\} \subset[c, d] \subset(0,1 / L),\left\{\lambda_{i, n}\right\} \subset\left[a_{i}, b_{i}\right] \subset\left(0,2 \eta_{i}\right)$, $i \in\{1,2, \ldots, N\}, \alpha_{n} \rightarrow 0, \gamma_{n} \rightarrow 0$, and $c_{n} \rightarrow 0$, we conclude from (68) and the boundedness of $\left\{x_{n}\right\},\left\{k_{n}\right\}$ that

$$
\lim _{n \rightarrow \infty}\left\|A_{i} \Omega_{n}^{i-1} u_{n}-A_{i} p\right\|=0, \quad i=1,2, \ldots, N
$$

By Proposition 4 and Lemma 12, we obtain

$$
\begin{aligned}
& \left\|\Omega_{n}^{i} u_{n}-p\right\|^{2} \\
& \quad=\left\|P_{C}\left(I-\lambda_{i, n} A_{i}\right) \Omega_{n}^{i-1} u_{n}-P_{C}\left(I-\lambda_{i, n} A_{i}\right) p\right\|^{2} \\
& \quad \leq\left\langle\left(I-\lambda_{i, n} A_{i}\right) \Omega_{n}^{i-1} u_{n}-\left(I-\lambda_{i, n} A_{i}\right) p, \Omega_{n}^{i} u_{n}-p\right\rangle
\end{aligned}
$$




$$
\begin{aligned}
& =\frac{1}{2}\left(\left\|\left(I-\lambda_{i, n} A_{i}\right) \Omega_{n}^{i-1} u_{n}-\left(I-\lambda_{i, n} A_{i}\right) p\right\|^{2}\right. \\
& +\left\|\Omega_{n}^{i} u_{n}-p\right\|^{2} \\
& \text { - } \|\left(I-\lambda_{i, n} A_{i}\right) \Omega_{n}^{i-1} u_{n} \\
& \left.-\left(I-\lambda_{i, n} A_{i}\right) p-\left(\Omega_{n}^{i} u_{n}-p\right) \|^{2}\right) \\
& \leq \frac{1}{2}\left(\left\|\Omega_{n}^{i-1} u_{n}-p\right\|^{2}+\left\|\Omega_{n}^{i} u_{n}-p\right\|^{2}\right. \\
& \left.-\left\|\Omega_{n}^{i-1} u_{n}-\Omega_{n}^{i} u_{n}-\lambda_{i, n}\left(A_{i} \Omega_{n}^{i-1} u_{n}-A_{i} p\right)\right\|^{2}\right) \\
& \leq \frac{1}{2}\left(\left\|u_{n}-p\right\|^{2}+\left\|\Omega_{n}^{i} u_{n}-p\right\|^{2}\right. \\
& \left.-\left\|\Omega_{n}^{i-1} u_{n}-\Omega_{n}^{i} u_{n}-\lambda_{i, n}\left(A_{i} \Omega_{n}^{i-1} u_{n}-A_{i} p\right)\right\|^{2}\right) \\
& \leq \frac{1}{2}\left(\left\|x_{n}-p\right\|^{2}+\left\|\Omega_{n}^{i} u_{n}-p\right\|^{2}\right. \\
& \left.-\left\|\Omega_{n}^{i-1} u_{n}-\Omega_{n}^{i} u_{n}-\lambda_{i, n}\left(A_{i} \Omega_{n}^{i-1} u_{n}-A_{i} p\right)\right\|^{2}\right),
\end{aligned}
$$

which implies

$$
\begin{aligned}
\left\|\Omega_{n}^{i} u_{n}-p\right\|^{2} \leq & \left\|x_{n}-p\right\|^{2} \\
& -\left\|\Omega_{n}^{i-1} u_{n}-\Omega_{n}^{i} u_{n}-\lambda_{i, n}\left(A_{i} \Omega_{n}^{i-1} u_{n}-A_{i} p\right)\right\|^{2} \\
= & \left\|x_{n}-p\right\|^{2}-\left\|\Omega_{n}^{i-1} u_{n}-\Omega_{n}^{i} u_{n}\right\|^{2} \\
& -\lambda_{i, n}^{2}\left\|A_{i} \Omega_{n}^{i-1} u_{n}-A_{i} p\right\|^{2} \\
& +2 \lambda_{i, n}\left\langle\Omega_{n}^{i-1} u_{n}-\Omega_{n}^{i} u_{n}, A_{i} \Omega_{n}^{i-1} u_{n}-A_{i} p\right\rangle \\
\leq & \left\|x_{n}-p\right\|^{2}-\left\|\Omega_{n}^{i-1} u_{n}-\Omega_{n}^{i} u_{n}\right\|^{2} \\
& +2 \lambda_{i, n}\left\langle\Omega_{n}^{i-1} u_{n}-\Omega_{n}^{i} u_{n}, A_{i} \Omega_{n}^{i-1} u_{n}-A_{i} p\right\rangle .
\end{aligned}
$$

Utilizing (81) and (85), we get

$$
\begin{aligned}
\left\|k_{n}-p\right\|^{2} \leq & \left\|\Omega_{n}^{i} u_{n}-p\right\|^{2}+2 \lambda_{n} \alpha_{n}\|p\| \\
& \times\left[\left\|x_{n}-p\right\|+\lambda_{n} \alpha_{n}\|p\|\right] \\
& +\gamma_{n}\left(\left\|x_{n}-p\right\|+\sqrt{2} \lambda_{n} \alpha_{n}\|p\|\right)^{2}+c_{n} \\
\leq & \left\|x_{n}-p\right\|^{2}-\left\|\Omega_{n}^{i-1} u_{n}-\Omega_{n}^{i} u_{n}\right\|^{2} \\
& +2 \lambda_{i, n}\left\langle\Omega_{n}^{i-1} u_{n}-\Omega_{n}^{i} u_{n}, A_{i} \Omega_{n}^{i-1} u_{n}-A_{i} p\right\rangle \\
& +2 \lambda_{n} \alpha_{n}\|p\|\left[\left\|x_{n}-p\right\|+\lambda_{n} \alpha_{n}\|p\|\right] \\
& +\gamma_{n}\left(\left\|x_{n}-p\right\|+\sqrt{2} \lambda_{n} \alpha_{n}\|p\|\right)^{2}+c_{n},
\end{aligned}
$$

which implies

$$
\begin{aligned}
\left\|\Omega_{n}^{i-1} u_{n}-\Omega_{n}^{i} u_{n}\right\|^{2} \\
\leq\left\|x_{n}-p\right\|^{2}-\left\|k_{n}-p\right\|^{2} \\
\quad+2 \lambda_{i, n}\left\langle\Omega_{n}^{i-1} u_{n}-\Omega_{n}^{i} u_{n}, A_{i} \Omega_{n}^{i-1} u_{n}-A_{i} p\right\rangle \\
\quad+2 \lambda_{n} \alpha_{n}\|p\|\left[\left\|x_{n}-p\right\|+\lambda_{n} \alpha_{n}\|p\|\right] \\
\quad+\gamma_{n}\left(\left\|x_{n}-p\right\|+\sqrt{2} \lambda_{n} \alpha_{n}\|p\|\right)^{2}+c_{n} \\
\leq\left\|x_{n}-k_{n}\right\|\left(\left\|x_{n}-p\right\|+\left\|k_{n}-p\right\|\right) \\
\quad+2 \lambda_{i, n}\left\|\Omega_{n}^{i-1} u_{n}-\Omega_{n}^{i} u_{n}\right\|\left\|A_{i} \Omega_{n}^{i-1} u_{n}-A_{i} p\right\| \\
\quad+2 \lambda_{n} \alpha_{n}\|p\|\left[\left\|x_{n}-p\right\|+\lambda_{n} \alpha_{n}\|p\|\right] \\
\quad+\gamma_{n}\left(\left\|x_{n}-p\right\|+\sqrt{2} \lambda_{n} \alpha_{n}\|p\|\right)^{2}+c_{n} .
\end{aligned}
$$

Since $\left\{\lambda_{n}\right\} \subset[c, d] \subset(0,1 / L),\left\{\lambda_{i, n}\right\} \subset\left[a_{i}, b_{i}\right] \subset\left(0,2 \eta_{i}\right)$, $i \in\{1,2, \ldots, N\}, \alpha_{n} \rightarrow 0, \gamma_{n} \rightarrow 0$, and $c_{n} \rightarrow 0$, we conclude from (68) and (83) and the boundedness of $\left\{x_{n}\right\},\left\{k_{n}\right\}$, and $\left\{u_{n}\right\}$ that

$$
\lim _{n \rightarrow \infty}\left\|\Omega_{n}^{i-1} u_{n}-\Omega_{n}^{i} u_{n}\right\|=0, \quad i=1,2, \ldots, N
$$

From (88) we get

$$
\begin{aligned}
\left\|u_{n}-\tilde{x}_{n}\right\|= & \left\|\Omega_{n}^{0} u_{n}-\Omega_{n}^{N} u_{n}\right\| \\
\leq & \left\|\Omega_{n}^{0} u_{n}-\Omega_{n}^{1} u_{n}\right\|+\left\|\Omega_{n}^{1} u_{n}-\Omega_{n}^{2} u_{n}\right\| \\
& +\cdots+\left\|\Omega_{n}^{N-1} u_{n}-\Omega_{n}^{N} u_{n}\right\|
\end{aligned}
$$

$$
\longrightarrow 0 \quad \text { as } n \longrightarrow \infty \text {. }
$$

By (79) and (89), we have

$$
\begin{aligned}
\left\|x_{n}-\tilde{x}_{n}\right\| & \leq\left\|x_{n}-u_{n}\right\|+\left\|u_{n}-\widetilde{x}_{n}\right\| \\
& \longrightarrow 0 \quad \text { as } n \longrightarrow \infty .
\end{aligned}
$$

From (64) and (90), we have

$$
\begin{aligned}
\left\|\tilde{x}_{n+1}-\tilde{x}_{n}\right\| \leq & \left\|\tilde{x}_{n+1}-x_{n+1}\right\| \\
& +\left\|x_{n+1}-x_{n}\right\|+\left\|x_{n}-\tilde{x}_{n}\right\|
\end{aligned}
$$


By (68), (79), and (89), we get

$$
\begin{aligned}
\left\|k_{n}-\tilde{x}_{n}\right\| & \leq\left\|k_{n}-x_{n}\right\|+\left\|x_{n}-u_{n}\right\|+\left\|u_{n}-\tilde{x}_{n}\right\| \\
& \longrightarrow 0 \quad \text { as } n \longrightarrow \infty .
\end{aligned}
$$

On the other hand, utilizing (58) and (59) we have

$$
\begin{aligned}
\left\|k_{n}-p\right\|^{2} \leq & {\left[1+\gamma_{n}\left(1-\delta_{n}\right)\right]\left\|z_{n}-p\right\|^{2} } \\
& +\left(1-\delta_{n}\right)\left(\kappa-\delta_{n}\right)\left\|z_{n}-S^{n} z_{n}\right\|^{2} \\
& +\left(1-\delta_{n}\right) c_{n} \\
\leq & \left\|z_{n}-p\right\|^{2}+\gamma_{n}\left\|z_{n}-p\right\|^{2}+c_{n} \\
\leq & \left\|\tilde{x}_{n}-p\right\|^{2}+2 \lambda_{n} \alpha_{n}\|p\|\left\|p-t_{n}\right\| \\
& +\left(\lambda_{n}^{2}\left(\alpha_{n}+L\right)^{2}-1\right)\left\|\tilde{x}_{n}-t_{n}\right\|^{2} \\
& +\gamma_{n}\left\|z_{n}-p\right\|^{2}+c_{n},
\end{aligned}
$$

which yields

$$
\begin{aligned}
(1 & \left.-d^{2}\left(\alpha_{n}+L\right)^{2}\right)\left\|\tilde{x}_{n}-t_{n}\right\|^{2} \\
\leq & \left(1-\lambda_{n}^{2}\left(\alpha_{n}+L\right)^{2}\right)\left\|\tilde{x}_{n}-t_{n}\right\|^{2} \\
\leq & \left\|\tilde{x}_{n}-p\right\|^{2}-\left\|k_{n}-p\right\|^{2} \\
& +2 \lambda_{n} \alpha_{n}\|p\|\left\|p-t_{n}\right\|+\gamma_{n}\left\|z_{n}-p\right\|^{2}+c_{n} \\
\leq & \left\|\tilde{x}_{n}-k_{n}\right\|\left(\left\|\tilde{x}_{n}-p\right\|+\left\|k_{n}-p\right\|\right) \\
& +2 \lambda_{n} \alpha_{n}\|p\|\left\|p-t_{n}\right\|+\gamma_{n}\left\|z_{n}-p\right\|^{2}+c_{n} .
\end{aligned}
$$

Since $\left\{\lambda_{n}\right\} \subset[c, d] \subset(0,1 / L), \alpha_{n} \rightarrow 0, \gamma_{n} \rightarrow 0$, and $c_{n} \rightarrow 0$, we conclude from (92) and the boundedness of $\left\{\tilde{x}_{n}\right\},\left\{k_{n}\right\},\left\{t_{n}\right\}$, and $\left\{z_{n}\right\}$ that

$$
\lim _{n \rightarrow \infty}\left\|\tilde{x}_{n}-t_{n}\right\|=0
$$

Also, utilizing the similar arguments to those of (58), we obtain

$$
\begin{aligned}
\| z_{n}- & p \|^{2} \\
\leq & \left\|\tilde{x}_{n}-p\right\|^{2}-\left\|\tilde{x}_{n}-t_{n}\right\|^{2} \\
& -\left\|t_{n}-z_{n}\right\|^{2}+2 \lambda_{n}\left(\alpha_{n}+L\right)\left\|\tilde{x}_{n}-t_{n}\right\|\left\|z_{n}-t_{n}\right\| \\
& +2 \lambda_{n} \alpha_{n}\left\langle p, p-t_{n}\right\rangle \\
\leq & \left\|\tilde{x}_{n}-p\right\|^{2}-\left\|\tilde{x}_{n}-t_{n}\right\|^{2}-\left\|t_{n}-z_{n}\right\|^{2} \\
& +\lambda_{n}^{2}\left(\alpha_{n}+L\right)^{2}\left\|t_{n}-z_{n}\right\|^{2}+\left\|\tilde{x}_{n}-t_{n}\right\|^{2} \\
& +2 \lambda_{n} \alpha_{n}\|p\|\left\|p-t_{n}\right\| \\
= & \left\|\tilde{x}_{n}-p\right\|^{2}+2 \lambda_{n} \alpha_{n}\|p\|\left\|p-t_{n}\right\| \\
& +\left(\lambda_{n}^{2}\left(\alpha_{n}+L\right)^{2}-1\right)\left\|t_{n}-z_{n}\right\|^{2},
\end{aligned}
$$

which together with (59) leads to

$$
\begin{aligned}
\| k_{n}- & p \|^{2} \\
\leq & {\left[1+\gamma_{n}\left(1-\delta_{n}\right)\right]\left\|z_{n}-p\right\|^{2} } \\
& +\left(1-\delta_{n}\right)\left(\kappa-\delta_{n}\right)\left\|z_{n}-S^{n} z_{n}\right\|^{2}+\left(1-\delta_{n}\right) c_{n} \\
\leq & \left\|z_{n}-p\right\|^{2}+\gamma_{n}\left\|z_{n}-p\right\|^{2}+c_{n} \\
\leq & \left\|\tilde{x}_{n}-p\right\|^{2}+2 \lambda_{n} \alpha_{n}\|p\|\left\|p-t_{n}\right\| \\
& +\left(\lambda_{n}^{2}\left(\alpha_{n}+L\right)^{2}-1\right)\left\|t_{n}-z_{n}\right\|^{2}+\gamma_{n}\left\|z_{n}-p\right\|^{2}+c_{n} .
\end{aligned}
$$

So we have

$$
\begin{aligned}
(1- & \left.d^{2}\left(\alpha_{n}+L\right)^{2}\right)\left\|t_{n}-z_{n}\right\|^{2} \\
\leq & \left(1-\lambda_{n}^{2}\left(\alpha_{n}+L\right)^{2}\right)\left\|t_{n}-z_{n}\right\|^{2} \\
\leq & \left\|\tilde{x}_{n}-p\right\|^{2}-\left\|k_{n}-p\right\|^{2} \\
& +2 \lambda_{n} \alpha_{n}\|p\|\left\|p-t_{n}\right\|+\gamma_{n}\left\|z_{n}-p\right\|^{2}+c_{n} \\
\leq & \left\|\tilde{x}_{n}-k_{n}\right\|\left(\left\|\tilde{x}_{n}-p\right\|+\left\|k_{n}-p\right\|\right) \\
& +2 \lambda_{n} \alpha_{n}\|p\|\left\|p-t_{n}\right\|+\gamma_{n}\left\|z_{n}-p\right\|^{2}+c_{n} .
\end{aligned}
$$

Since $\left\{\lambda_{n}\right\} \subset[c, d] \subset(0,1 / L), \alpha_{n} \rightarrow 0, \gamma_{n} \rightarrow 0$, and $c_{n} \rightarrow 0$, we conclude from (92) and the boundedness of $\left\{\tilde{x}_{n}\right\},\left\{k_{n}\right\},\left\{t_{n}\right\}$, and $\left\{z_{n}\right\}$ that

$$
\lim _{n \rightarrow \infty}\left\|t_{n}-z_{n}\right\|=0 .
$$

Utilizing (92)-(99), we get

$$
\begin{aligned}
\left\|k_{n}-z_{n}\right\| & \leq\left\|k_{n}-\tilde{x}_{n}\right\|+\left\|\tilde{x}_{n}-t_{n}\right\|+\left\|t_{n}-z_{n}\right\| \\
& \longrightarrow 0 \text { as } n \longrightarrow \infty .
\end{aligned}
$$

Since $k_{n}=\delta_{n} z_{n}+\left(1-\delta_{n}\right) S^{n} z_{n}$, we get

$$
(1-b)\left\|S^{n} z_{n}-z_{n}\right\| \leq\left(1-\delta_{n}\right)\left\|S^{n} z_{n}-z_{n}\right\|=\left\|k_{n}-z_{n}\right\|,
$$

which together with (100) implies

$$
\lim _{n \rightarrow \infty}\left\|S^{n} z_{n}-z_{n}\right\|=0
$$

In addition, observe that

$$
\left\|u_{n}-z_{n}\right\| \leq\left\|u_{n}-x_{n}\right\|+\left\|x_{n}-k_{n}\right\|+\left\|k_{n}-z_{n}\right\| .
$$

From (68), (79), and (100), it immediately follows that

$$
\lim _{n \rightarrow \infty}\left\|u_{n}-z_{n}\right\|=0 .
$$

Moreover, note that

$$
\begin{aligned}
\left\|z_{n+1}-z_{n}\right\| \leq & \left\|z_{n+1}-k_{n+1}\right\|+\left\|k_{n+1}-\tilde{x}_{n+1}\right\| \\
& +\left\|\tilde{x}_{n+1}-\tilde{x}_{n}\right\|+\left\|\tilde{x}_{n}-k_{n}\right\|+\left\|k_{n}-z_{n}\right\| .
\end{aligned}
$$


From (91), (92), and (100), it immediately follows that

$$
\lim _{n \rightarrow \infty}\left\|z_{n+1}-z_{n}\right\|=0
$$

Meantime, it is clear that

$$
\begin{aligned}
& \left\|S^{n} z_{n}-S^{n+1} z_{n}\right\| \leq\left\|S^{n} z_{n}-z_{n}\right\|+\left\|z_{n}-z_{n+1}\right\| \\
& \quad+\left\|z_{n+1}-S^{n+1} z_{n+1}\right\|+\left\|S^{n+1} z_{n+1}-S^{n+1} z_{n}\right\| .
\end{aligned}
$$

From (102) and (106) and Lemma 14, we obtain

$$
\lim _{n \rightarrow \infty}\left\|S^{n} z_{n}-S^{n+1} z_{n}\right\|=0
$$

Furthermore, we note that

$$
\begin{gathered}
\left\|z_{n}-S z_{n}\right\| \leq\left\|z_{n}-S^{n} z_{n}\right\|+\left\|S^{n} z_{n}-S^{n+1} z_{n}\right\| \\
+\left\|S^{n+1} z_{n}-S z_{n}\right\| .
\end{gathered}
$$

From (102) and (108) and the uniform continuity of $S$, we have

$$
\lim _{n \rightarrow \infty}\left\|z_{n}-S z_{n}\right\|=0
$$

Step 4. Finally we prove that $x_{n} \rightarrow v=P_{F} x_{0}$ as $n \rightarrow \infty$.

Indeed, since $\left\{x_{n}\right\}$ is bounded, there exists a subsequence $\left\{x_{n_{i}}\right\}$ which converges weakly to some $w$. From (70), (88)(90), and (104), we have that $\Theta_{n_{i}}^{k} x_{n_{i}} \rightarrow w, \Omega_{n_{i}}^{m} u_{n_{i}} \rightarrow w$, $z_{n_{i}} \rightarrow w, \tilde{x}_{n_{i}} \rightarrow w$, where $k \in\{1,2, \ldots, M\}, m \in$ $\{1,2, \ldots, N\}$. Since $S$ is uniformly continuous, by (110) we get $\lim _{n \rightarrow \infty}\left\|z_{n}-S^{m} z_{n}\right\|=0$ for any $m \geq 1$. Hence from Lemma 16, we obtain $w \in \operatorname{Fix}(S)$. Next we prove that $w \in$ $\cap_{m=1}^{N} \operatorname{VI}\left(C, A_{m}\right)$. Let

$$
T_{m} v= \begin{cases}A_{m} v+N_{C} v, & v \in C, \\ \emptyset, & v \notin C,\end{cases}
$$

where $m \in\{1,2, \ldots, N\}$. Let $(v, u) \in G\left(T_{m}\right)$. Since $u-A_{m} v \in$ $N_{C} v$ and $\Omega_{n}^{m} u_{n} \in C$, we have

$$
\left\langle v-\Omega_{n}^{m} u_{n}, u-A_{m} v\right\rangle \geq 0
$$

On the other hand, from $\Omega_{n}^{m} u_{n}=P_{C}\left(I-\lambda_{m, n} A_{m}\right) \Omega_{n}^{m-1} u_{n}$ and $v \in C$, we have

$$
\left\langle v-\Omega_{n}^{m} u_{n}, \Omega_{n}^{m} u_{n}-\left(\Omega_{n}^{m-1} u_{n}-\lambda_{m, n} A_{m} \Omega_{n}^{m-1} u_{n}\right)\right\rangle \geq 0,
$$

and hence

$$
\left\langle v-\Omega_{n}^{m} u_{n}, \frac{\Omega_{n}^{m} u_{n}-\Omega_{n}^{m-1} u_{n}}{\lambda_{m, n}}+A_{m} \Omega_{n}^{m-1} u_{n}\right\rangle \geq 0 .
$$

Therefore, we have

$$
\begin{aligned}
&\left\langle v-\Omega_{n_{i}}^{m} u_{n_{i}}, u\right\rangle \geq\left\langle v-\Omega_{n_{i}}^{m} u_{n_{i}}, A_{m} v\right\rangle \\
& \geq\left\langle v-\Omega_{n_{i}}^{m} u_{n_{i}}, A_{m} v\right\rangle \\
&-\left\langle v-\Omega_{n_{i}}^{m} u_{n_{i}}, \frac{\Omega_{n_{i}}^{m} u_{n_{i}}-\Omega_{n_{i}}^{m-1} u_{n_{i}}}{\lambda_{m, n_{i}}}\right. \\
&\left.+A_{m} \Omega_{n_{i}}^{m-1} u_{n_{i}}\right\rangle \\
&=\left\langle v-\Omega_{n_{i}}^{m} u_{n_{i}}, A_{m} v-A_{m} \Omega_{n_{i}}^{m} u_{n_{i}}\right\rangle \\
&+\left\langle v-\Omega_{n_{i}}^{m} u_{n_{i}}, A_{m} \Omega_{n_{i}}^{m} u_{n_{i}}-A_{m} \Omega_{n_{i}}^{m-1} u_{n_{i}}\right\rangle \\
&-\left\langle v-\Omega_{n_{i}}^{m} u_{n_{i}}, \frac{\Omega_{n_{i}}^{m} u_{n_{i}}-\Omega_{n_{i}}^{m-1} u_{n_{i}}}{\lambda_{m, n_{i}}}\right\rangle \\
& \geq\left\langle v-\Omega_{n_{i}}^{m} u_{n_{i}}, A_{m} \Omega_{n_{i}}^{m} u_{n_{i}}-A_{m} \Omega_{n_{i}}^{m-1} u_{n_{i}}\right\rangle \\
&-\left\langle v-\Omega_{n_{i}}^{m} u_{n_{i}}, \frac{\Omega_{n_{i}}^{m} u_{n_{i}}-\Omega_{n_{i}}^{m-1} u_{n_{i}}}{\lambda_{m, n_{i}}}\right\rangle . \\
&\left\langle\begin{array}{l}
\langle \\
\geq
\end{array}\right.
\end{aligned}
$$

From (88) and since $A_{m}$ is continuous, we obtain that $\lim _{n \rightarrow \infty}\left\|A_{m} \Omega_{n}^{m} u_{n}-A_{m} \Omega_{n}^{m-1} u_{n}\right\|=0$. From $\Omega_{n_{i}}^{m} u_{n_{i}} \rightarrow w$, $\left\{\lambda_{i, n}\right\} \subset\left[a_{i}, b_{i}\right] \subset\left(0,2 \eta_{i}\right)$, for all $i \in\{1,2, \ldots, N\}$ and (88), we have

$$
\langle v-w, u\rangle \geq 0
$$

Since $T_{m}$ is maximal monotone, we have $w \in T_{m}^{-1} 0$ and hence $w \in \operatorname{VI}\left(C, A_{m}\right), m=1,2, \ldots, N$, which implies $w \in \cap_{m=1}^{N} \operatorname{VI}\left(C, A_{m}\right)$. Next we prove that $w \in \cap_{k=1}^{M} \operatorname{GMEP}\left(F_{k}, \varphi_{k}, B_{k}\right)$. Since $\Theta_{n}^{k} x_{n}=T_{r_{k, n}}^{\left(F_{k}, \varphi_{k}\right)}(I-$ $\left.r_{k, n} B_{k}\right) \Theta_{n}^{k-1} x_{n}, n \geq 1, k \in\{1,2, \ldots, M\}$, we have

$$
\begin{gathered}
F_{k}\left(\Theta_{n}^{k} x_{n}, y\right)+\varphi_{k}(y)-\varphi_{k}\left(\Theta_{n}^{k} x_{n}\right)+\left\langle B_{k} \Theta_{n}^{k-1} x_{n}, y-\Theta_{n}^{k} x_{n}\right\rangle \\
+\frac{1}{r_{k, n}}\left\langle y-\Theta_{n}^{k} x_{n}, \Theta_{n}^{k} x_{n}-\Theta_{n}^{k-1} x_{n}\right\rangle \geq 0 .
\end{gathered}
$$

By (A2), we have

$$
\begin{aligned}
\varphi_{k}(y) & -\varphi_{k}\left(\Theta_{n}^{k} x_{n}\right)+\left\langle B_{k} \Theta_{n}^{k-1} x_{n}, y-\Theta_{n}^{k} x_{n}\right\rangle \\
& +\frac{1}{r_{k, n}}\left\langle y-\Theta_{n}^{k} x_{n}, \Theta_{n}^{k} x_{n}-\Theta_{n}^{k-1} x_{n}\right\rangle \geq F_{k}\left(y, \Theta_{n}^{k} x_{n}\right) .
\end{aligned}
$$


Let $z_{t}=t y+(1-t) w$ for all $t \in(0,1]$ and $y \in C$. This implies that $z_{t} \in C$. Then we have

$$
\begin{aligned}
\left\langle z_{t}\right. & \left.-\Theta_{n}^{k} x_{n}, B_{k} z_{t}\right\rangle \\
\geq & \varphi_{k}\left(\Theta_{n}^{k} x_{n}\right)-\varphi_{k}\left(z_{t}\right)+\left\langle z_{t}-\Theta_{n}^{k} x_{n}, B_{k} z_{t}\right\rangle \\
& -\left\langle z_{t}-\Theta_{n}^{k} x_{n}, B_{k} \Theta_{n}^{k-1} x_{n}\right\rangle \\
& -\left\langle z_{t}-\Theta_{n}^{k} x_{n}, \frac{\Theta_{n}^{k} x_{n}-\Theta_{n}^{k-1} x_{n}}{r_{k, n}}\right\rangle+F_{k}\left(z_{t}, \Theta_{n}^{k} x_{n}\right) \\
= & \varphi_{k}\left(\Theta_{n}^{k} x_{n}\right)-\varphi_{k}\left(z_{t}\right)+\left\langle z_{t}-\Theta_{n}^{k} x_{n}, B_{k} z_{t}-B_{k} \Theta_{n}^{k} x_{n}\right\rangle \\
& +\left\langle z_{t}-\Theta_{n}^{k} x_{n}, B_{k} \Theta_{n}^{k} x_{n}-B_{k} \Theta_{n}^{k-1} x_{n}\right\rangle \\
& -\left\langle z_{t}-\Theta_{n}^{k} x_{n}, \frac{\Theta_{n}^{k} x_{n}-\Theta_{n}^{k-1} x_{n}}{r_{k, n}}\right\rangle+F_{k}\left(z_{t}, \Theta_{n}^{k} x_{n}\right) .
\end{aligned}
$$

By (70), we have $\left\|B_{k} \Theta_{n}^{k} x_{n}-B_{k} \Theta_{n}^{k-1} x_{n}\right\| \rightarrow 0$ as $n \rightarrow \infty$. Furthermore, by the monotonicity of $B_{k}$, we obtain $\left\langle z_{t}-\right.$ $\left.\Theta_{n}^{k} x_{n}, B_{k} z_{t}-B_{k} \Theta_{n}^{k} x_{n}\right\rangle \geq 0$. Then by (A4), we obtain

$$
\left\langle z_{t}-w, B_{k} z_{t}\right\rangle \geq \varphi_{k}(w)-\varphi_{k}\left(z_{t}\right)+F_{k}\left(z_{t}, w\right) .
$$

Utilizing (A1), (A4), and (120), we obtain

$$
\begin{aligned}
0= & F_{k}\left(z_{t}, z_{t}\right)+\varphi_{k}\left(z_{t}\right)-\varphi_{k}\left(z_{t}\right) \\
\leq & t F_{k}\left(z_{t}, y\right)+(1-t) F_{k}\left(z_{t}, w\right) \\
& +t \varphi_{k}(y)+(1-t) \varphi_{k}(w)-\varphi_{k}\left(z_{t}\right) \\
\leq & t\left[F_{k}\left(z_{t}, y\right)+\varphi_{k}(y)-\varphi_{k}\left(z_{t}\right)\right] \\
& +(1-t)\left\langle z_{t}-w, B_{k} z_{t}\right\rangle \\
= & t\left[F_{k}\left(z_{t}, y\right)+\varphi_{k}(y)-\varphi_{k}\left(z_{t}\right)\right] \\
& +(1-t) t\left\langle y-w, B_{k} z_{t}\right\rangle
\end{aligned}
$$

and hence

$$
0 \leq F_{k}\left(z_{t}, y\right)+\varphi_{k}(y)-\varphi_{k}\left(z_{t}\right)+(1-t)\left\langle y-w, B_{k} z_{t}\right\rangle .
$$

Letting $t \rightarrow 0$, we have, for each $y \in C$,

$$
0 \leq F_{k}(w, y)+\varphi_{k}(y)-\varphi_{k}(w)+\left\langle y-w, B_{k} w\right\rangle
$$

This implies that $w \in \operatorname{GMEP}\left(F_{k}, \varphi_{k}, B_{k}\right)$ and hence $w \in$ $\cap_{k=1}^{M} \operatorname{GMEP}\left(F_{k}, \varphi_{k}, B_{k}\right)$.

Further, let us show that $w \in \Gamma$. As a matter of fact, since $\tilde{x}_{n}-t_{n} \rightarrow 0$ and $\tilde{x}_{n}-z_{n} \rightarrow 0$ (due to (95) and (99)), we have $t_{n_{i}} \rightarrow w$ and $z_{n_{i}} \rightarrow w$. Let

$$
T v= \begin{cases}\nabla f(v)+N_{C} v, & \text { if } v \in C, \\ \emptyset, & \text { if } v \notin C,\end{cases}
$$

where $N_{C} v$ is the normal cone to $C$ at $v \in C$. We have already mentioned that in this case the mapping $T$ is maximal monotone, and $0 \in T v$ if and only if $v \in \operatorname{VI}(C, \nabla f)$; see [40] for more details. Let $G(T)$ be the graph of $T$ and let $(v, u) \in G(T)$. Then, we have $u \in T v=\nabla f(v)+N_{C} v$ and hence $u-\nabla f(v) \in N_{C} v$. So we have $\langle v-t, u-\nabla f(v)\rangle \geq 0$ for all $t \in C$. On the other hand, from $z_{n}=P_{C}\left(\tilde{x}_{n}-\lambda_{n} \nabla f_{\alpha_{n}}\left(t_{n}\right)\right)$ and $v \in C$, we have

$$
\left\langle\tilde{x}_{n}-\lambda_{n} \nabla f_{\alpha_{n}}\left(t_{n}\right)-z_{n}, z_{n}-v\right\rangle \geq 0,
$$

and hence

$$
\left\langle v-z_{n}, \frac{z_{n}-\tilde{x}_{n}}{\lambda_{n}}+\nabla f_{\alpha_{n}}\left(t_{n}\right)\right\rangle \geq 0 .
$$

Therefore, from $\langle v-t, u-\nabla f(v)\rangle \geq 0$ for all $t \in C$ and $z_{n_{i}} \in C$, we have

$$
\begin{aligned}
\left\langle v-z_{n_{i}}, u\right\rangle \geq & \left\langle v-z_{n_{i}}, \nabla f(v)\right\rangle \\
\geq & \left\langle v-z_{n_{i}}, \nabla f(v)\right\rangle \\
& -\left\langle v-z_{n_{i}}, \frac{z_{n_{i}}-\tilde{x}_{n_{i}}}{\lambda_{n_{i}}}+\nabla f_{\alpha_{n_{i}}}\left(t_{n_{i}}\right)\right\rangle \\
= & \left\langle v-z_{n_{i}}, \nabla f(v)\right\rangle \\
& -\left\langle v-z_{n_{i}}, \frac{z_{n_{i}}-\tilde{x}_{n_{i}}}{\lambda_{n_{i}}}+\nabla f\left(t_{n_{i}}\right)\right\rangle \\
& -\alpha_{n_{i}}\left\langle v-z_{n_{i}}, t_{n_{i}}\right\rangle \\
= & \left\langle v-z_{n_{i}}, \nabla f(v)-\nabla f\left(z_{n_{i}}\right)\right\rangle \\
& +\left\langle v-z_{n_{i}}, \nabla f\left(z_{n_{i}}\right)-\nabla f\left(t_{n_{i}}\right)\right\rangle \\
& -\left\langle v-z_{n_{i}}, \frac{z_{n_{i}}-\tilde{x}_{n_{i}}}{\lambda_{n_{i}}}\right\rangle-\alpha_{n_{i}}\left\langle v-z_{n_{i}}, t_{n_{i}}\right\rangle \\
\geq & \left\langle v-z_{n_{i}}, \nabla f\left(z_{n_{i}}\right)-\nabla f\left(t_{n_{i}}\right)\right\rangle \\
& -\left\langle v-z_{n_{i}}, \frac{z_{n_{i}}-\tilde{x}_{n_{i}}}{\lambda_{n_{i}}}\right\rangle-\alpha_{n_{i}}\left\langle v-z_{n_{i}}, t_{n_{i}}\right\rangle .
\end{aligned}
$$

Note that $\alpha_{n} \rightarrow 0,\left\|z_{n}-\tilde{x}_{n}\right\| \rightarrow 0$, and $\left\|\nabla f\left(z_{n}\right)-\nabla f\left(t_{n}\right)\right\| \rightarrow$ 0 (due to the $L$-Lipschitz continuity of $\nabla f$ ). Thus, we obtain $\langle v-w, u\rangle \geq 0$ as $i \rightarrow \infty$. Since $T$ is maximal monotone, we have $w \in T^{-1} 0$ and hence $w \in \operatorname{VI}(C, \nabla f)$. Clearly, $w \in \Gamma$. Consequently, $w \in F$. This shows that $\omega_{w}\left(\left\{x_{n}\right\}\right) \subset F$. From (61) and Lemma 23 we infer that $x_{n} \rightarrow v=P_{F} x_{0}$ as $n \rightarrow \infty$. This completes the proof.

Corollary 25 (i.e., [3, Theorem 3.1]). Let $C$ be a nonempty closed convex subset of a real Hilbert space $H$. Let $f$ : $C \rightarrow \mathbf{R}$ be a convex functional with L-Lipschitz continuous gradient $\nabla f$. Let $M, N$ be two integers. Let $F_{k}$ be a bifunction from $C \times C$ to $\mathbf{R}$ satisfying $(A 1)-(A 4)$ and let $\varphi_{k}: C \rightarrow$ $\mathbf{R} \cup\{+\infty\}$ be a proper lower semicontinuous and convex 
function, where $k \in\{1,2, \ldots, M\}$. Let $B_{k}$ and let $A_{i}$ be $\mu_{k^{-}}$ inverse strongly monotone and $\eta_{i}$-inverse-strongly monotone, respectively, where $k \in\{1,2, \ldots, M\}, i \in\{1,2, \ldots, N\}$. Let $S: C \rightarrow C$ be a uniformly continuous asymptotically $\kappa$-strict pseudocontractive mapping in the intermediate sense for some $0 \leq \kappa<1$ with sequence $\left\{\gamma_{n}\right\} \subset[0, \infty)$ such that $\lim _{n \rightarrow \infty} \gamma_{n}=$ 0 and $\left\{c_{n}\right\} \subset[0, \infty)$ such that $\lim _{n \rightarrow \infty} c_{n}=0$. Assume that $F:=\cap_{k=1}^{M} \operatorname{GMEP}\left(F_{k}, \varphi_{k}, A_{k}\right) \cap \cap_{i=1}^{N} \operatorname{VI}\left(C, A_{i}\right) \cap \operatorname{Fix}(S) \cap \Gamma$ is nonempty and bounded. Let $\left\{\beta_{n}\right\}$ and $\left\{\delta_{n}\right\}$ be sequences in $[0,1]$ such that $0<a \leq \beta_{n} \leq 1$ and $\kappa \leq \delta_{n} \leq b<1$. Pick any $x_{0} \in H$ and set $C_{1}=C, x_{1}=P_{C_{1}} x_{0}$. Let $\left\{x_{n}\right\}$ be a sequence generated by the following algorithm:

$$
\begin{gathered}
u_{n}=T_{r_{M, n}}^{\left(F_{M}, \varphi_{M}\right)}\left(I-r_{M, n} B_{M}\right) T_{r_{M, n}}^{\left(F_{M-1}, \varphi_{M-1}\right)} \\
\times\left(I-r_{M-1, n} B_{M-1}\right) \cdots T_{r_{1, n}}^{\left(F_{1}, \varphi_{1}\right)}\left(I-r_{1, n} B_{1}\right) x_{n}, \\
z_{n}=P_{C}\left(I-\lambda_{N, n} A_{N}\right) P_{C}\left(I-\lambda_{N-1, n} A_{N-1}\right) \\
\cdots P_{C}\left(I-\lambda_{2, n} A_{2}\right) P_{C}\left(I-\lambda_{1, n} A_{1}\right) u_{n}, \\
k_{n}=\delta_{n} z_{n}+\left(1-\delta_{n}\right) S^{n} z_{n}, \\
y_{n}=\left(1-\beta_{n}\right) x_{n}+\beta_{n} k_{n}, \\
C_{n+1}=\left\{z \in C_{n}:\left\|y_{n}-z\right\|^{2} \leq\left\|x_{n}-z\right\|^{2}+\theta_{n}\right\} \\
x_{n+1}=P_{C_{n+1}} x_{0}, \quad \forall n \geq 0
\end{gathered}
$$

where $\theta_{n}=\gamma_{n} \Delta_{n}^{2}+c_{n}, \Delta_{n}=\sup \left\{\left\|x_{n}-p\right\|: p \in F\right\}<\infty$, $\left\{\lambda_{i, n}\right\} \subset\left[a_{i}, b_{i}\right] \subset\left(0,2 \eta_{i}\right),\left\{r_{k, n}\right\} \subset\left[e_{k}, f_{k}\right] \subset\left(0,2 \mu_{k}\right), i \in$ $\{1,2, \ldots, N\}, k \in\{1,2, \ldots, M\}$. Assume that either (B1) or (B2) holds. Then $\left\{x_{n}\right\}$ converge strongly to $P_{F} x_{0}$.

Proof. In Theorem 24, put $\nabla f=0$ and $\alpha_{n}=0$ for all $n \geq 1$. Then $\Gamma=C$ and $F=\cap_{k=1}^{M} \operatorname{GMEP}\left(F_{k}, \varphi_{k}, A_{k}\right) \cap$ $\cap_{i=1}^{N} \operatorname{VI}\left(C, A_{i}\right) \cap \operatorname{Fix}(S) \cap \Gamma=\cap_{k=1}^{M} \operatorname{GMEP}\left(F_{k}, \varphi_{k}, A_{k}\right) \cap$ $\cap_{i=1}^{N} \operatorname{VI}\left(C, A_{i}\right) \cap \operatorname{Fix}(S)$. In this case, we obtain from (44) that

$$
\begin{aligned}
& t_{n}=P_{C}\left(\tilde{x}_{n}-\lambda_{n} \nabla f_{\alpha_{n}}\left(\tilde{x}_{n}\right)\right)=\tilde{x}_{n}, \\
& z_{n}=P_{C}\left(\tilde{x}_{n}-\lambda_{n} \nabla f_{\alpha_{n}}\left(t_{n}\right)\right)=\tilde{x}_{n} .
\end{aligned}
$$

Thus, the iterative scheme (44) reduces to (128). Since $\alpha_{n}=0$ for all $n \geq 1$ and $F$ is bounded, we know that $\varrho=\sup \{\|p\|$ : $p \in F\}<\infty, \Delta_{n}=\sup \left\{\left\|x_{n}-p\right\|: p \in F\right\}<\infty$, and

$$
\theta_{n}=\left(\gamma_{n}+\lambda_{n} \alpha_{n}\right) \Delta_{n}^{2}+3 \lambda_{n} \alpha_{n}\left(1+\gamma_{n}\right)^{2} \varrho^{2}+c_{n}=\gamma_{n} \Delta_{n}^{2}+c_{n} .
$$

It is easy to see that all the conditions of Theorem 24 are satisfied. Therefore, in terms of Theorem 24, we derive the desired result.

Corollary 26. Let $C$ be a nonempty closed convex subset of a real Hilbert space $H$. Let $f: C \rightarrow \mathbf{R}$ be a convex functional with L-Lipschitz continuous gradient $\nabla f$. Let $F$ be a bifunction from $C \times C$ to $\mathbf{R}$ satisfying (A1)-(A4) and let $\varphi$ : $C \rightarrow \mathbf{R} \cup\{+\infty\}$ be a proper lower semicontinuous and convex function. Let $B$ and $A_{i}$ be $\mu$-inverse strongly monotone and $\eta_{i}$-inverse-strongly monotone, respectively, where $i=1,2$. Let $S: C \rightarrow C$ be a uniformly continuous asymptotically $\kappa$-strict pseudocontractive mapping in the intermediate sense for some $0 \leq \kappa<1$ with sequence $\left\{\gamma_{n}\right\} \subset[0, \infty)$ such that $\lim _{n \rightarrow \infty} \gamma_{n}=$ 0 and $\left\{c_{n}\right\} \subset[0, \infty)$ such that $\lim _{n \rightarrow \infty} c_{n}=0$. Assume that $F:=\operatorname{GMEP}(F, \varphi, A) \cap \operatorname{VI}\left(C, A_{1}\right) \cap \operatorname{VI}\left(C, A_{2}\right) \cap \operatorname{Fix}(S) \cap \Gamma$ is nonempty and bounded. Let $\left\{\alpha_{n}\right\}$ be a sequence in $[0, \infty)$, and let $\left\{\beta_{n}\right\},\left\{\delta_{n}\right\}$ be sequences in $[0,1]$ such that $0<a \leq \beta_{n} \leq 1$ and $\kappa \leq \delta_{n} \leq b<1$. Pick any $x_{0} \in H$ and set $C_{1}=C$, $x_{1}=P_{C_{1}} x_{0}$. Let $\left\{x_{n}\right\}$ be a sequence generated by the following algorithm:

$$
\begin{gathered}
F\left(u_{n}, y\right)+\varphi(y)-\varphi\left(u_{n}\right)+\left\langle B x_{n}, y-u_{n}\right\rangle \\
+\frac{1}{r_{n}}\left\langle y-u_{n}, u_{n}-x_{n}\right\rangle \geq 0, \quad \forall y \in C, \\
\tilde{x}_{n}=P_{C}\left(I-\lambda_{2, n} A_{2}\right) P_{C}\left(I-\lambda_{1, n} A_{1}\right) u_{n}, \\
t_{n}=P_{C}\left(\tilde{x}_{n}-\lambda_{n} \nabla f_{\alpha_{n}}\left(\tilde{x}_{n}\right)\right), \\
z_{n}=P_{C}\left(\tilde{x}_{n}-\lambda_{n} \nabla f_{\alpha_{n}}\left(t_{n}\right)\right), \\
k_{n}=\delta_{n} z_{n}+\left(1-\delta_{n}\right) S^{n} z_{n}, \\
y_{n}=\left(1-\beta_{n}\right) x_{n}+\beta_{n} k_{n}, \\
C_{n+1}=\left\{z \in C_{n}:\left\|y_{n}-z\right\|^{2} \leq\left\|x_{n}-z\right\|^{2}+\theta_{n}\right\}, \\
x_{n+1}=P_{C_{n+1}} x_{0}, \quad \forall n \geq 0,
\end{gathered}
$$

where $\theta_{n}=\left(\gamma_{n}+\lambda_{n} \alpha_{n}\right) \Delta_{n}^{2}+3 \lambda_{n} \alpha_{n}\left(1+\gamma_{n}\right)^{2} \varrho^{2}+c_{n}, \Delta_{n}=$ $\sup \left\{\left\|x_{n}-p\right\|: p \in F\right\}<\infty$ and $\varrho=\sup \{\|p\|: p \in F\}<\infty$. Assume that the following conditions hold:

(i) $\lim _{n \rightarrow \infty} \alpha_{n}=0$;

(ii) $\left\{\lambda_{n}\right\} \subset[c, d]$ for some $c, d \in(0,1 / L)$;

(iii) $\left\{\lambda_{i, n}\right\} \subset\left[a_{i}, b_{i}\right] \subset\left(0,2 \eta_{i}\right), i=1,2$;

(iv) $\left\{r_{n}\right\} \subset[e, f] \subset(0,2 \mu)$.

Then $\left\{x_{n}\right\}$ converge strongly to $P_{F} x_{0}$ provided either (B1) or (B2) holds.

Corollary 27. Let $C$ be a nonempty closed convex subset of a real Hilbert space $H$. Let $f: C \rightarrow \mathbf{R}$ be a convex functional with L-Lipschitz continuous gradient $\nabla f$. Let $F$ be a bifunction from $C \times C$ to $\mathbf{R}$ satisfying (A1)-(A4) and let $\varphi: C \rightarrow \mathbf{R} \cup$ $\{+\infty\}$ be a proper lower semicontinuous and convex function. Let $B$ and $A$ be $\mu$-inverse strongly monotone and $\eta$-inversestrongly monotone, respectively. Let $S: C \rightarrow C$ be a uniformly continuous asymptotically $\kappa$-strict pseudocontractive mapping in the intermediate sense for some $0 \leq \kappa<1$ with sequence $\left\{\gamma_{n}\right\} \subset[0, \infty)$ such that $\lim _{n \rightarrow \infty} \gamma_{n}=0$ and $\left\{c_{n}\right\} \subset[0, \infty)$ such that $\lim _{n \rightarrow \infty} c_{n}=0$. Assume that $F:=\operatorname{GMEP}(F, \varphi, A) \cap$ $\operatorname{VI}(C, A) \cap \operatorname{Fix}(S) \cap \Gamma$ is nonempty and bounded. Let $\left\{\alpha_{n}\right\}$ be a sequence in $[0, \infty)$, and let $\left\{\beta_{n}\right\},\left\{\delta_{n}\right\}$ be sequences in $[0,1]$ such that $0<a \leq \beta_{n} \leq 1$ and $\kappa \leq \delta_{n} \leq b<1$. Pick any $x_{0} \in H$ 
and set $C_{1}=C, x_{1}=P_{C_{1}} x_{0}$. Let $\left\{x_{n}\right\}$ be a sequence generated by the following algorithm:

$$
\begin{gathered}
F\left(u_{n}, y\right)+\varphi(y)-\varphi\left(u_{n}\right)+\left\langle B x_{n}, y-u_{n}\right\rangle \\
+\frac{1}{r_{n}}\left\langle y-u_{n}, u_{n}-x_{n}\right\rangle \geq 0, \quad \forall y \in C, \\
\tilde{x}_{n}=P_{C}\left(u_{n}-\rho_{n} A u_{n}\right), \\
t_{n}=P_{C}\left(\tilde{x}_{n}-\lambda_{n} \nabla f_{\alpha_{n}}\left(\tilde{x}_{n}\right)\right), \\
z_{n}=P_{C}\left(\tilde{x}_{n}-\lambda_{n} \nabla f_{\alpha_{n}}\left(t_{n}\right)\right), \\
k_{n}=\delta_{n} z_{n}+\left(1-\delta_{n}\right) S^{n} z_{n}, \\
y_{n}=\left(1-\beta_{n}\right) x_{n}+\beta_{n} k_{n}, \\
C_{n+1}=\left\{z \in C_{n}:\left\|y_{n}-z\right\|^{2} \leq\left\|x_{n}-z\right\|^{2}+\theta_{n}\right\}, \\
x_{n+1}=P_{C_{n+1}} x_{0}, \quad \forall n \geq 0,
\end{gathered}
$$

where $\theta_{n}=\left(\gamma_{n}+\lambda_{n} \alpha_{n}\right) \Delta_{n}^{2}+3 \lambda_{n} \alpha_{n}\left(1+\gamma_{n}\right)^{2} \varrho^{2}+c_{n}, \Delta_{n}=$ $\sup \left\{\left\|x_{n}-p\right\|: p \in F\right\}<\infty$, and $\varrho=\sup \{\|p\|: p \in F\}<\infty$. Assume that the following conditions hold:

(i) $\lim _{n \rightarrow \infty} \alpha_{n}=0$;

(ii) $\left\{\lambda_{n}\right\} \subset[c, d]$ for some $c, d \in(0,1 / L)$;

(iii) $\left\{\rho_{n}\right\} \subset[a, b] \subset(0,2 \eta)$;

(iv) $\left\{r_{n}\right\} \subset[e, f] \subset(0,2 \mu)$.

Then $\left\{x_{n}\right\}$ converge strongly to $P_{F} x_{0}$ provided either (B1) or (B2) holds.

Corollary 28. Let $C$ be a nonempty closed convex subset of a real Hilbert space $H$. Let $f: C \rightarrow \mathbf{R}$ be a convex functional with L-Lipschitz continuous gradient $\nabla f$. Let $F$ be a bifunction from $C \times C$ to $\mathbf{R}$ satisfying (A1)-(A4) and let $\varphi: C \rightarrow \mathbf{R} \cup$ $\{+\infty\}$ be a proper lower semicontinuous and convex function. Let $B$ and $A$ be $\mu$-inverse strongly monotone and $\eta$-inversestrongly monotone, respectively. Let $S: C \rightarrow C$ be a uniformly continuous asymptotically $\kappa$-strict pseudocontractive mapping for some $0 \leq \kappa<1$ with sequence $\left\{\gamma_{n}\right\} \subset[0, \infty)$ such that $\lim _{n \rightarrow \infty} \gamma_{n}=0$. Assume that $F:=\operatorname{GMEP}(F, \varphi, A) \cap \operatorname{VI}(C, A) \cap$ $\operatorname{Fix}(S) \cap \Gamma$ is nonempty and bounded. Let $\left\{\alpha_{n}\right\}$ be a sequence in $[0, \infty)$, and let $\left\{\beta_{n}\right\},\left\{\delta_{n}\right\}$ be sequences in $[0,1]$ such that $0<a \leq \beta_{n} \leq 1$ and $\kappa \leq \delta_{n} \leq b<1$. Pick any $x_{0} \in H$ and set $C_{1}=C, x_{1}=P_{C_{1}} x_{0}$. Let $\left\{x_{n}\right\}$ be a sequence generated by the following algorithm:

$$
\begin{gathered}
F\left(u_{n}, y\right)+\varphi(y)-\varphi\left(u_{n}\right)+\left\langle B x_{n}, y-u_{n}\right\rangle \\
+\frac{1}{r_{n}}\left\langle y-u_{n}, u_{n}-x_{n}\right\rangle \geq 0, \quad \forall y \in C, \\
\tilde{x}_{n}=P_{C}\left(u_{n}-\rho_{n} A u_{n}\right), \\
t_{n}=P_{C}\left(\tilde{x}_{n}-\lambda_{n} \nabla f_{\alpha_{n}}\left(\tilde{x}_{n}\right)\right),
\end{gathered}
$$

$$
\begin{gathered}
z_{n}=P_{C}\left(\tilde{x}_{n}-\lambda_{n} \nabla f_{\alpha_{n}}\left(t_{n}\right)\right), \\
k_{n}=\delta_{n} z_{n}+\left(1-\delta_{n}\right) S^{n} z_{n}, \\
y_{n}=\left(1-\beta_{n}\right) x_{n}+\beta_{n} k_{n}, \\
C_{n+1}=\left\{z \in C_{n}:\left\|y_{n}-z\right\|^{2} \leq\left\|x_{n}-z\right\|^{2}+\theta_{n}\right\}, \\
x_{n+1}=P_{C_{n+1}} x_{0}, \quad \forall n \geq 0,
\end{gathered}
$$

where $\theta_{n}=\left(\gamma_{n}+\lambda_{n} \alpha_{n}\right) \Delta_{n}^{2}+3 \lambda_{n} \alpha_{n}\left(1+\gamma_{n}\right)^{2} \varrho^{2}, \Delta_{n}=\sup \left\{\| x_{n}-\right.$ $p \|: p \in F\}<\infty$, and $\varrho=\sup \{\|p\|: p \in F\}<\infty$. Assume that the following conditions hold:

(i) $\lim _{n \rightarrow \infty} \alpha_{n}=0$;

(ii) $\left\{\lambda_{n}\right\} \subset[c, d]$ for some $c, d \in(0,1 / L)$;

(iii) $\left\{\rho_{n}\right\} \subset[a, b] \subset(0,2 \eta)$;

(iv) $\left\{r_{n}\right\} \subset[e, f] \subset(0,2 \mu)$.

Then $\left\{x_{n}\right\}$ converge strongly to $P_{F} x_{0}$ provided either (B1) or (B2) holds.

Remark 29. Theorem 24 extends, improves, supplements, and develops Yao et al. [26, Theorem 3.1] in the following aspects.

(i) Theorem 24 generalizes and extends [26, Theorem 3.1] from the asymptotically $k$-strict pseudocontractive mapping to the asymptotically $k$-strict pseudocontractive mapping in the intermediate sense and from one mixed equilibrium problem to finite GMEPs.

(ii) We add finite VIPs and the CMP (2) in our algorithm such that it can be applied to find a common element of the set of solutions of finite GMEPs, the set of solutions of finite VIPs for inverse strongly monotone mappings, the set of fixed points of an asymptotically $k$-strict pseudocontractive mapping in the intermediate sense, and the CMP (2) for a convex functional $f$ with $L$-Lipschitz continuous gradient $\nabla f$.

(iii) Theorem 24 also removes the condition (ii) in [26, Theorem 3.1].

\section{Weak Convergence Theorem}

In this section, we prove a new weak convergence theorem by a modified extragradient method with regularization for finding a common element of the set of solutions of the CMP (2) for a convex functional $f: C \rightarrow \mathbf{R}$ with $L$ Lipschitz continuous gradient $\nabla f$, the set of solutions of finite generalized mixed equilibrium problems, the set of solutions of finite variational inequalities for inverse strong monotone mappings, and the set of fixed points of an asymptotically $\kappa$-strict pseudocontractive mapping $S: C \rightarrow C$ in the intermediate sense in a real Hilbert space. This iterative algorithm with regularization is based on the extragradient method, Mann-type iterative method, and hybrid gradient projection algorithm (GPA) with regularization. 
Theorem 30. Let $C$ be a nonempty closed convex subset of a real Hilbert space $H$. Let $f: C \rightarrow \mathbf{R}$ be a convex functional with L-Lipschitz continuous gradient $\nabla f$. Let $M, N$ be two integers. Let $F_{k}$ be a bifunction from $C \times C$ to $\mathbf{R}$ satisfying (A1)-(A4) and let $\varphi_{k}: C \rightarrow \mathbf{R} \cup\{+\infty\}$ be a proper lower semicontinuous and convex function, where $k \in\{1,2, \ldots, M\}$. Let $B_{k}$ and $A_{i}$ be $\mu_{k}$-inverse strongly monotone and $\eta_{i}$-inversestrongly monotone, respectively, where $k \in\{1,2, \ldots, M\}$, $i \in\{1,2, \ldots, N\}$. Let $S: C \rightarrow C$ be the uniformly continuous asymptotically $\kappa$-strict pseudocontractive mapping in the intermediate sense for some $0 \leq \kappa<1$ with sequences $\left\{\gamma_{n}\right\} \subset[0, \infty)$ and $\left\{c_{n}\right\} \subset[0, \infty)$. Assume that $F:=\cap_{k=1}^{M} \operatorname{GMEP}\left(F_{k}, \varphi_{k}, A_{k}\right) \cap \cap_{i=1}^{N} \operatorname{VI}\left(C, A_{i}\right) \cap \operatorname{Fix}(S) \cap \Gamma$ is nonempty. Let $\left\{\alpha_{n}\right\}$ be a sequence in $(0, \infty)$, and let $\left\{\beta_{n}\right\},\left\{\delta_{n}\right\}$ be sequences in $[0,1]$ such that $0<a \leq \beta_{n} \leq \beta<1$ and $\kappa \leq \delta_{n} \leq b<1$. Pick any $x_{1} \in H$ and let $\left\{x_{n}\right\}$ be a sequence generated by the following algorithm:

$$
\begin{gathered}
u_{n}=T_{r_{M, n}}^{\left(F_{M}, \varphi_{M}\right)}\left(I-r_{M, n} B_{M}\right) T_{r_{M, n}}^{\left(F_{M-1}, \varphi_{M-1}\right)} \\
\times\left(I-r_{M-1, n} B_{M-1}\right) \cdots T_{r_{1, n}}^{\left(F_{1}, \varphi_{1}\right)}\left(I-r_{1, n} B_{1}\right) x_{n}, \\
\tilde{x}_{n}=P_{C}\left(I-\lambda_{N, n} A_{N}\right) P_{C}\left(I-\lambda_{N-1, n} A_{N-1}\right) \\
\cdots P_{C}\left(I-\lambda_{2, n} A_{2}\right) P_{C}\left(I-\lambda_{1, n} A_{1}\right) u_{n}, \\
t_{n}=P_{C}\left(\tilde{x}_{n}-\lambda_{n} \nabla f_{\alpha_{n}}\left(\tilde{x}_{n}\right)\right), \\
z_{n}=P_{C}\left(\tilde{x}_{n}-\lambda_{n} \nabla f_{\alpha_{n}}\left(t_{n}\right)\right), \\
k_{n}=\delta_{n} z_{n}+\left(1-\delta_{n}\right) S^{n} z_{n}, \\
x_{n+1}=\left(1-\beta_{n}\right) x_{n}+\beta_{n} k_{n},
\end{gathered}
$$

where $\left\{\lambda_{i, n}\right\} \subset\left[a_{i}, b_{i}\right] \subset\left(0,2 \eta_{i}\right),\left\{r_{k, n}\right\} \subset\left[e_{k}, f_{k}\right] \subset\left(0,2 \mu_{k}\right), i \in$ $\{1,2, \ldots, N\}$, and $k \in\{1,2, \ldots, M\}$. Assume that either (B1) or (B2) holds and that the following conditions are satisfied:

(i) $\sum_{n=1}^{\infty} \alpha_{n}<\infty, \sum_{n=1}^{\infty} \gamma_{n}<\infty$ and $\sum_{n=1}^{\infty} c_{n}<\infty$;

(ii) $\left\{\lambda_{n}\right\} \subset[c, d]$ for some $c, d \in(0,1 / L)$.

Then

(a) $\left\{x_{n}\right\}$ converges weakly to an element $w \in F$;

(b) $\left\{x_{n}\right\}$ converges weakly to $w=\lim _{n \rightarrow \infty} P_{F} x_{n}$ provided $F$ is bounded.

Proof. First of all, again one can show that $P_{C}\left(I-\lambda \nabla f_{\alpha}\right)$ is $\xi$-averaged for each $\lambda \in(0,2 /(\alpha+L))$, where

$$
\xi=\frac{2+\lambda(\alpha+L)}{4} \in(0,1) .
$$

This shows that $P_{C}\left(I-\lambda \nabla f_{\alpha}\right)$ is nonexpansive. Furthermore, for $\left\{\lambda_{n}\right\} \subset[a, b]$ with $a, b \in(0,1 / L)$, without loss of generality, we may assume that

$$
a \leq \inf _{n \geq 1} \lambda_{n} \leq \sup _{n \geq 1} \lambda_{n} \leq b<\frac{1}{\alpha_{n}+L}, \quad \forall n \geq 1 .
$$

Consequently, it follows that for each integer $n \geq 1, P_{C}(I-$ $\left.\lambda_{n} \nabla f_{\alpha_{n}}\right)$ is $\xi_{n}$-averaged with

$$
\begin{aligned}
\xi_{n} & =\frac{1}{2}+\frac{\lambda_{n}\left(\alpha_{n}+L\right)}{2}-\frac{1}{2} \cdot \frac{\lambda_{n}\left(\alpha_{n}+L\right)}{2} \\
& =\frac{2+\lambda_{n}\left(\alpha_{n}+L\right)}{4} \in(0,1) .
\end{aligned}
$$

This immediately implies that $P_{C}\left(I-\lambda_{n} \nabla f_{\alpha_{n}}\right)$ is nonexpansive for all $n \geq 1$.

Next let us show that $\lim _{n \rightarrow \infty}\left\|x_{n}-p\right\|$ exists for any $p \in F$. Put

$$
\begin{aligned}
\Theta_{n}^{k}= & T_{r_{k, n}}^{\left(F_{k}, \varphi_{k}\right)}\left(I-r_{k, n} B_{k}\right) T_{r_{k-1, n}}^{\left(F_{k-1}, \varphi_{k-1}\right)} \\
& \times\left(I-r_{k-1, n} B_{M-1}\right) \cdots T_{r_{1, n}}^{\left(F_{1}, \varphi_{1}\right)}\left(I-r_{1, n} B_{1}\right) x_{n},
\end{aligned}
$$

for all $k \in\{1,2, \ldots, M\}, n \geq 1$, and $\Theta_{n}^{0}=\Omega_{n}^{0}=I$, where $I$ is the identity mapping on $H$. Then we have that $u_{n}=\Theta_{n}^{M} x_{n}$ and $\widetilde{x}_{n}=\Omega_{n}^{N} u_{n}$. Take $p \in F$ arbitrarily. Similarly to the proof of Theorem 24, we obtain that

$$
\begin{gathered}
\left\|u_{n}-p\right\| \leq\left\|x_{n}-p\right\|, \\
\left\|\tilde{x}_{n}-p\right\| \leq\left\|u_{n}-p\right\|, \\
\left\|t_{n}-p\right\| \leq\left\|x_{n}-p\right\|+\lambda_{n} \alpha_{n}\|p\| \\
\left\|z_{n}-p\right\|^{2} \leq\left\|\tilde{x}_{n}-p\right\|^{2}+2 \lambda_{n} \alpha_{n}\|p\|\left\|p-t_{n}\right\| \\
+\left(\lambda_{n}^{2}\left(\alpha_{n}+L\right)^{2}-1\right)\left\|\tilde{x}_{n}-t_{n}\right\|^{2} \\
\leq\left\|\tilde{x}_{n}-p\right\|^{2}+2 \lambda_{n} \alpha_{n}\|p\|\left\|p-t_{n}\right\| \\
\leq\left\|x_{n}-p\right\|^{2}+2 \lambda_{n} \alpha_{n}\|p\|\left\|x_{n}-p\right\| \\
+2 \lambda_{n}^{2} \alpha_{n}^{2}\|p\|^{2}, \\
\left\|z_{n}-p\right\|^{2} \leq\left\|\tilde{x}_{n}-p\right\|^{2}+2 \lambda_{n} \alpha_{n}\|p\|\left\|p-t_{n}\right\| \\
+\left(\lambda_{n}^{2}\left(\alpha_{n}+L\right)^{2}-1\right)\left\|t_{n}-z_{n}\right\|^{2}, \\
\left\|\Theta_{n}^{k} x_{n}-p\right\|^{2} \leq\left\|x_{n}-p\right\|^{2}+r_{k, n}\left(r_{k, n}-2 \mu_{k}\right) \\
\times
\end{gathered}
$$




$$
\begin{aligned}
\left\|\Theta_{n}^{k} x_{n}-p\right\|^{2} \leq & \left\|x_{n}-p\right\|^{2}-\left\|\Theta_{n}^{k-1} x_{n}-\Theta_{n}^{k} x_{n}\right\|^{2} \\
& +2 r_{k, n}\left\|\Theta_{n}^{k-1} x_{n}-\Theta_{n}^{k} x_{n}\right\|\left\|B_{k} \Theta_{n}^{k-1} x_{n}-B_{k} p\right\|, \\
k=1,2, \ldots, M, & (144)
\end{aligned}
$$

$$
\begin{array}{r}
\left\|\Omega_{n}^{i} u_{n}-p\right\|^{2} \leq\left\|x_{n}-p\right\|^{2}-\left\|\Omega_{n}^{i-1} u_{n}-\Omega_{n}^{i} u_{n}\right\|^{2} \\
+2 \lambda_{i, n}\left\|\Omega_{n}^{i-1} u_{n}-\Omega_{n}^{i} u_{n}\right\|\left\|A_{i} \Omega_{n}^{i-1} u_{n}-A_{i} p\right\|, \\
i \in\{1,2, \ldots, N\} .
\end{array}
$$

We observe that

$$
\begin{aligned}
\left\|k_{n}-p\right\|^{2}= & \left\|\delta_{n}\left(z_{n}-p\right)+\left(1-\delta_{n}\right)\left(S^{n} z_{n}-p\right)\right\|^{2} \\
= & \delta_{n}\left\|z_{n}-p\right\|^{2}+\left(1-\delta_{n}\right)\left\|S^{n} z_{n}-p\right\|^{2} \\
& -\delta_{n}\left(1-\delta_{n}\right)\left\|z_{n}-S^{n} z_{n}\right\|^{2} \\
\leq & \delta_{n}\left\|z_{n}-p\right\|^{2}+\left(1-\delta_{n}\right) \\
& \times\left[\left(1+\gamma_{n}\right)\left\|z_{n}-p\right\|^{2}+\kappa\left\|z_{n}-S^{n} z_{n}\right\|^{2}+c_{n}\right] \\
& -\delta_{n}\left(1-\delta_{n}\right)\left\|z_{n}-S^{n} z_{n}\right\|^{2} \\
= & {\left[1+\gamma_{n}\left(1-\delta_{n}\right)\right]\left\|z_{n}-p\right\|^{2} } \\
& +\left(1-\delta_{n}\right)\left(\kappa-\delta_{n}\right)\left\|z_{n}-S^{n} z_{n}\right\|^{2}+\left(1-\delta_{n}\right) c_{n} \\
\leq & \left(1+\gamma_{n}\right)\left\|z_{n}-p\right\|^{2}+c_{n} .
\end{aligned}
$$

It follows from (142) and (147) and $\lambda_{n}\left(\alpha_{n}+L\right)<1$ that

$$
\begin{aligned}
& \left\|x_{n+1}-p\right\|^{2} \\
& =\left\|\left(1-\beta_{n}\right)\left(x_{n}-p\right)+\beta_{n}\left(k_{n}-p\right)\right\|^{2} \\
& \leq\left(1-\beta_{n}\right)\left\|x_{n}-p\right\|^{2}+\beta_{n}\left\|k_{n}-p\right\|^{2} \\
& \leq\left(1-\beta_{n}\right)\left\|x_{n}-p\right\|^{2}+\beta_{n}\left[\left(1+\gamma_{n}\right)\left\|z_{n}-p\right\|^{2}+c_{n}\right] \\
& \leq\left(1-\beta_{n}\right)\left\|x_{n}-p\right\|^{2} \\
& \quad+\beta_{n}\left\{( 1 + \gamma _ { n } ) \left[\left\|x_{n}-p\right\|^{2}+2 \lambda_{n} \alpha_{n}\|p\|\left\|x_{n}-p\right\|\right.\right. \\
& \left.\left.\quad+2 \lambda_{n}^{2} \alpha_{n}^{2}\|p\|^{2}\right]+c_{n}\right\}
\end{aligned}
$$

$$
\begin{aligned}
\leq & \left(1+\gamma_{n}\right)\left[\left\|x_{n}-p\right\|^{2}+2 \lambda_{n} \alpha_{n}\|p\|\left\|x_{n}-p\right\|\right. \\
& \left.+2 \lambda_{n}^{2} \alpha_{n}^{2}\|p\|^{2}\right]+c_{n} \\
= & \left(1+\gamma_{n}\right)\left\|x_{n}-p\right\|^{2}+2\left(1+\gamma_{n}\right) \lambda_{n} \alpha_{n}\|p\|\left\|x_{n}-p\right\| \\
& +2\left(1+\gamma_{n}\right) \lambda_{n}^{2} \alpha_{n}^{2}\|p\|^{2}+c_{n} \\
\leq & \left(1+\gamma_{n}\right)\left\|x_{n}-p\right\|^{2}+\left(1+\gamma_{n}\right) \lambda_{n} \alpha_{n}\left(\|p\|^{2}+\left\|x_{n}-p\right\|^{2}\right) \\
& +2\left(1+\gamma_{n}\right) \lambda_{n} \alpha_{n}\|p\|^{2}+c_{n} \\
= & {\left[1+\gamma_{n}+\left(1+\gamma_{n}\right) \lambda_{n} \alpha_{n}\right]\left\|x_{n}-p\right\|^{2} } \\
& +3\left(1+\gamma_{n}\right) \lambda_{n} \alpha_{n}\|p\|^{2}+c_{n} .
\end{aligned}
$$

From $\left\{\lambda_{n}\right\} \subset[c, d] \subset(0,1 / L)$ and condition (i) we have

$$
\begin{gathered}
\sum_{n=1}^{\infty}\left[\gamma_{n}+\left(1+\gamma_{n}\right) \lambda_{n} \alpha_{n}\right]<\infty, \\
\sum_{n=1}^{\infty}\left[3\left(1+\gamma_{n}\right) \lambda_{n} \alpha_{n}\|p\|^{2}+c_{n}\right]<\infty .
\end{gathered}
$$

So, applying Lemma 19 to (148), we deduce that $\lim _{n \rightarrow \infty} \| x_{n}-$ $p \|$ exists. This implies that $\left\{x_{n}\right\}$ is bounded and hence $\left\{u_{n}\right\},\left\{\tilde{x}_{n}\right\},\left\{t_{n}\right\},\left\{z_{n}\right\}$, and $\left\{k_{n}\right\}$ are also bounded. In addition, by Lemma 12 and (59) we obtain from $\lambda_{n}\left(\alpha_{n}+L\right)<1$ that

$$
\begin{aligned}
\left\|x_{n+1}-p\right\|^{2}= & \left(1-\beta_{n}\right)\left\|x_{n}-p\right\|^{2}+\beta_{n}\left\|k_{n}-p\right\|^{2} \\
& -\beta_{n}\left(1-\beta_{n}\right)\left\|x_{n}-k_{n}\right\|^{2} \\
\leq & \left(1-\beta_{n}\right)\left\|x_{n}-p\right\|^{2} \\
& +\beta_{n}\left\{( 1 + \gamma _ { n } ) \left[\left\|x_{n}-p\right\|^{2}\right.\right. \\
& +2 \lambda_{n} \alpha_{n}\|p\|\left\|x_{n}-p\right\| \\
& \left.\left.+2 \lambda_{n}^{2} \alpha_{n}^{2}\|p\|^{2}\right]+c_{n}\right\} \\
& -\beta_{n}\left(1-\beta_{n}\right)\left\|x_{n}-k_{n}\right\|^{2} \\
\leq & \left(1+\gamma_{n}\right)\left[\left\|x_{n}-p\right\|^{2}+2 \lambda_{n} \alpha_{n}\|p\|\right. \\
& \left.\times\left\|x_{n}-p\right\|+2 \lambda_{n}^{2} \alpha_{n}^{2}\|p\|^{2}\right] \\
& +c_{n}-\beta_{n}\left(1-\beta_{n}\right)\left\|x_{n}-k_{n}\right\|^{2} \\
\leq & \left(1+\gamma_{n}\right)\left[\left\|x_{n}-p\right\|^{2}+2 \lambda_{n} \alpha_{n}\|p\|\left\|x_{n}-p\right\|\right. \\
& \left.+2 \lambda_{n} \alpha_{n}\|p\|^{2}\right]+c_{n} \\
& -\beta_{n}\left(1-\beta_{n}\right)\left\|x_{n}-k_{n}\right\|^{2}
\end{aligned}
$$




$$
\begin{aligned}
= & \left(1+\gamma_{n}\right)\left\|x_{n}-p\right\|^{2}+2\left(1+\gamma_{n}\right) \lambda_{n} \alpha_{n} \\
& \times\|p\|\left(\left\|x_{n}-p\right\|+\|p\|\right) \\
& +c_{n}-\beta_{n}\left(1-\beta_{n}\right)\left\|x_{n}-k_{n}\right\|^{2} .
\end{aligned}
$$

Thus, it is easy to see from $0<a \leq \beta_{n} \leq \beta<1$ that

$$
\begin{aligned}
& a(1-\beta)\left\|x_{n}-k_{n}\right\|^{2} \\
& \leq \beta_{n}\left(1-\beta_{n}\right)\left\|x_{n}-k_{n}\right\|^{2} \\
& \leq\left\|x_{n}-p\right\|^{2}-\left\|x_{n+1}-p\right\|^{2}+\gamma_{n}\left\|x_{n}-p\right\|^{2} \\
& \quad+2\left(1+\gamma_{n}\right) \lambda_{n} \alpha_{n}\|p\|\left(\left\|x_{n}-p\right\|+\|p\|\right)+c_{n} .
\end{aligned}
$$

Since $\left\{\lambda_{n}\right\} \subset[c, d] \subset(0,1 / L), \lim _{n \rightarrow \infty}\left\|x_{n}-p\right\|$ exists, $\alpha_{n} \rightarrow$ $0, \gamma_{n} \rightarrow 0, c_{n} \rightarrow 0$, and the sequence $\left\{x_{n}\right\}$ is bounded, we obtain

$$
\lim _{n \rightarrow \infty}\left\|x_{n}-k_{n}\right\|=0 .
$$

Taking into consideration $x_{n+1}=\left(1-\beta_{n}\right) x_{n}+\beta_{n} k_{n}$, we get $x_{n+1}-x_{n}=\beta_{n}\left(k_{n}-x_{n}\right)$, which together with (152) leads to

$$
\lim _{n \rightarrow \infty}\left\|x_{n+1}-x_{n}\right\|=0 .
$$

Combining (140), (142), (143), and (147), we have

$$
\begin{aligned}
&\left\|k_{n}-p\right\|^{2} \\
& \leq\left\|z_{n}-p\right\|^{2}+\gamma_{n}\left\|z_{n}-p\right\|^{2}+c_{n} \\
& \leq\left\|\tilde{x}_{n}-p\right\|^{2}+2 \lambda_{n} \alpha_{n}\|p\|\left\|p-t_{n}\right\|+\gamma_{n}\left\|z_{n}-p\right\|^{2}+c_{n} \\
& \leq\left\|u_{n}-p\right\|^{2}+2 \lambda_{n} \alpha_{n}\|p\|\left\|p-t_{n}\right\|+\gamma_{n}\left\|z_{n}-p\right\|^{2}+c_{n} \\
& \leq\left\|\Theta_{n}^{k} x_{n}-p\right\|^{2}+2 \lambda_{n} \alpha_{n}\|p\|\left\|p-t_{n}\right\|+\gamma_{n}\left\|z_{n}-p\right\|^{2}+c_{n} \\
& \leq\left\|x_{n}-p\right\|^{2}+r_{k, n}\left(r_{k, n}-2 \mu_{k}\right)\left\|B_{k} \Theta_{n}^{k-1} x_{n}-B_{k} p\right\|^{2} \\
&+2 \lambda_{n} \alpha_{n}\|p\|\left\|p-t_{n}\right\|+\gamma_{n}\left\|z_{n}-p\right\|^{2}+c_{n},
\end{aligned}
$$

which yields

$$
\begin{aligned}
& r_{k, n}\left(2 \mu_{k}-r_{k, n}\right)\left\|B_{k} \Theta_{n}^{k-1} x_{n}-B_{k} p\right\|^{2} \\
& \leq\left\|x_{n}-p\right\|^{2}-\left\|k_{n}-p\right\|^{2}+2 \lambda_{n} \alpha_{n}\|p\|\left\|p-t_{n}\right\| \\
&+\gamma_{n}\left\|z_{n}-p\right\|^{2}+c_{n} \\
& \leq\left\|x_{n}-k_{n}\right\|\left(\left\|x_{n}-p\right\|+\left\|k_{n}-p\right\|\right) \\
&+2 \lambda_{n} \alpha_{n}\|p\|\left\|p-t_{n}\right\|+\gamma_{n}\left\|z_{n}-p\right\|^{2}+c_{n} .
\end{aligned}
$$

Since $\left\{\lambda_{n}\right\} \subset[c, d] \subset(0,1 / L),\left\{r_{k, n}\right\} \subset\left[e_{k}, f_{k}\right] \subset\left(0,2 \mu_{k}\right), k \in$ $\{1,2, \ldots, M\}, \alpha_{n} \rightarrow 0, \gamma_{n} \rightarrow 0$, and $c_{n} \rightarrow 0$, we conclude from (152) and the boundedness of $\left\{x_{n}\right\},\left\{k_{n}\right\},\left\{t_{n}\right\}$, and $\left\{z_{n}\right\}$ that

$$
\lim _{n \rightarrow \infty}\left\|B_{k} \Theta_{n}^{k-1} x_{n}-B_{k} p\right\|=0, \quad k=1,2, \ldots, M
$$

Combining (140), (142), (144), and (147), we have

$$
\begin{aligned}
\| k_{n}- & p \|^{2} \\
\leq & \left\|\Theta_{n}^{k} x_{n}-p\right\|^{2}+2 \lambda_{n} \alpha_{n}\|p\|\left\|p-t_{n}\right\| \\
& +\gamma_{n}\left\|z_{n}-p\right\|^{2}+c_{n} \\
\leq & \left\|x_{n}-p\right\|^{2}-\left\|\Theta_{n}^{k-1} x_{n}-\Theta_{n}^{k} x_{n}\right\|^{2} \\
& +2 r_{k, n}\left\|\Theta_{n}^{k-1} x_{n}-\Theta_{n}^{k} x_{n}\right\|\left\|B_{k} \Theta_{n}^{k-1} x_{n}-B_{k} p\right\| \\
& +2 \lambda_{n} \alpha_{n}\|p\|\left\|p-t_{n}\right\|+\gamma_{n}\left\|z_{n}-p\right\|^{2}+c_{n},
\end{aligned}
$$

which implies

$$
\begin{aligned}
\| \Theta_{n}^{k-1} & x_{n}-\Theta_{n}^{k} x_{n} \|^{2} \\
\leq & \left\|x_{n}-p\right\|^{2}-\left\|k_{n}-p\right\|^{2} \\
& +2 r_{k, n}\left\|\Theta_{n}^{k-1} x_{n}-\Theta_{n}^{k} x_{n}\right\|\left\|B_{k} \Theta_{n}^{k-1} x_{n}-B_{k} p\right\| \\
& +2 \lambda_{n} \alpha_{n}\|p\|\left\|p-t_{n}\right\|+\gamma_{n}\left\|z_{n}-p\right\|^{2}+c_{n} \\
\leq & \left\|x_{n}-k_{n}\right\|\left(\left\|x_{n}-p\right\|+\left\|k_{n}-p\right\|\right) \\
& +2 r_{k, n}\left\|\Theta_{n}^{k-1} x_{n}-\Theta_{n}^{k} x_{n}\right\|\left\|B_{k} \Theta_{n}^{k-1} x_{n}-B_{k} p\right\| \\
& +2 \lambda_{n} \alpha_{n}\|p\|\left\|p-t_{n}\right\|+\gamma_{n}\left\|z_{n}-p\right\|^{2}+c_{n} .
\end{aligned}
$$

Since $\left\{\lambda_{n}\right\} \subset[c, d] \subset(0,(1 / L)),\left\{r_{k, n}\right\} \subset\left[e_{k}, f_{k}\right] \subset\left(0,2 \mu_{k}\right)$, $k \in\{1,2, \ldots, M\}, \alpha_{n} \rightarrow 0, \gamma_{n} \rightarrow 0$, and $c_{n} \rightarrow 0$, we conclude from (152) and (156) and the boundedness of $\left\{x_{n}\right\},\left\{k_{n}\right\},\left\{t_{n}\right\}$, and $\left\{z_{n}\right\}$ that

$$
\lim _{n \rightarrow \infty}\left\|\Theta_{n}^{k-1} x_{n}-\Theta_{n}^{k} x_{n}\right\|=0, \quad k=1,2, \ldots, M
$$

From (159), we have

$$
\begin{aligned}
\left\|x_{n}-u_{n}\right\|= & \left\|\Theta_{n}^{0} x_{n}-\Theta_{n}^{M} x_{n}\right\| \\
\leq & \left\|\Theta_{n}^{0} x_{n}-\Theta_{n}^{1} x_{n}\right\|+\left\|\Theta_{n}^{1} x_{n}-\Theta_{n}^{2} x_{n}\right\| \\
& +\cdots+\left\|\Theta_{n}^{M-1} x_{n}-\Theta_{n}^{M} x_{n}\right\|
\end{aligned}
$$


Combining (140), (142), (145), and (147), we have

$$
\begin{aligned}
\| k_{n}- & p \|^{2} \\
\leq & \left\|z_{n}-p\right\|^{2}+\gamma_{n}\left\|z_{n}-p\right\|^{2}+c_{n} \\
\leq & \left\|\tilde{x}_{n}-p\right\|^{2}+2 \lambda_{n} \alpha_{n}\|p\|\left\|p-t_{n}\right\| \\
& +\gamma_{n}\left\|z_{n}-p\right\|^{2}+c_{n} \\
\leq & \left\|\Omega_{n}^{i} u_{n}-p\right\|^{2}+2 \lambda_{n} \alpha_{n}\|p\|\left\|p-t_{n}\right\| \\
& +\gamma_{n}\left\|z_{n}-p\right\|^{2}+c_{n} \\
\leq & \left\|x_{n}-p\right\|^{2}+\lambda_{i, n}\left(\lambda_{i, n}-2 \eta_{i}\right)\left\|A_{i} \Omega_{n}^{i-1} u_{n}-A_{i} p\right\|^{2} \\
& +2 \lambda_{n} \alpha_{n}\|p\|\left\|p-t_{n}\right\|+\gamma_{n}\left\|z_{n}-p\right\|^{2}+c_{n},
\end{aligned}
$$

where $i \in\{1,2, \ldots, N\}$, which implies

$$
\begin{aligned}
& \lambda_{i, n}\left(2 \eta_{i}-\lambda_{i, n}\right)\left\|A_{i} \Omega_{n}^{i-1} u_{n}-A_{i} p\right\|^{2} \\
& \leq\left\|x_{n}-p\right\|^{2}-\left\|k_{n}-p\right\|^{2} \\
&+2 \lambda_{n} \alpha_{n}\|p\|\left\|p-t_{n}\right\|+\gamma_{n}\left\|z_{n}-p\right\|^{2}+c_{n} \\
& \leq\left\|x_{n}-k_{n}\right\|\left(\left\|x_{n}-p\right\|+\left\|k_{n}-p\right\|\right) \\
&+2 \lambda_{n} \alpha_{n}\|p\|\left\|p-t_{n}\right\|+\gamma_{n}\left\|z_{n}-p\right\|^{2}+c_{n} .
\end{aligned}
$$

Since $\left\{\lambda_{n}\right\} \subset[c, d] \subset(0,1 / L),\left\{\lambda_{i, n}\right\} \subset\left[a_{i}, b_{i}\right] \subset\left(0,2 \eta_{i}\right), i \in$ $\{1,2, \ldots, N\}, \alpha_{n} \rightarrow 0, \gamma_{n} \rightarrow 0$, and $c_{n} \rightarrow 0$, we conclude from (152) and the boundedness of $\left\{x_{n}\right\},\left\{k_{n}\right\},\left\{t_{n}\right\}$, and $\left\{z_{n}\right\}$ that

$$
\lim _{n \rightarrow \infty}\left\|A_{i} \Omega_{n}^{i-1} u_{n}-A_{i} p\right\|=0, \quad i \in\{1,2, \ldots, N\}
$$

Combining (140), (142), (146), and (147), we have

$$
\begin{aligned}
\| k_{n}- & p \|^{2} \\
\leq & \left\|z_{n}-p\right\|^{2}+\gamma_{n}\left\|z_{n}-p\right\|^{2}+c_{n} \\
\leq & \left\|\tilde{x}_{n}-p\right\|^{2}+2 \lambda_{n} \alpha_{n}\|p\|\left\|p-t_{n}\right\| \\
& +\gamma_{n}\left\|z_{n}-p\right\|^{2}+c_{n} \\
\leq & \left\|\Omega_{n}^{i} u_{n}-p\right\|^{2}+2 \lambda_{n} \alpha_{n}\|p\|\left\|p-t_{n}\right\| \\
& +\gamma_{n}\left\|z_{n}-p\right\|^{2}+c_{n} \\
\leq & \left\|x_{n}-p\right\|^{2}-\left\|\Omega_{n}^{i-1} u_{n}-\Omega_{n}^{i} u_{n}\right\|^{2} \\
& +2 \lambda_{i, n}\left\|\Omega_{n}^{i-1} u_{n}-\Omega_{n}^{i} u_{n}\right\|\left\|A_{i} \Omega_{n}^{i-1} u_{n}-A_{i} p\right\| \\
& +2 \lambda_{n} \alpha_{n}\|p\|\left\|p-t_{n}\right\|+\gamma_{n}\left\|z_{n}-p\right\|^{2}+c_{n},
\end{aligned}
$$

which implies

$$
\begin{aligned}
&\left\|\Omega_{n}^{i-1} u_{n}-\Omega_{n}^{i} u_{n}\right\|^{2} \\
& \leq\left\|x_{n}-p\right\|^{2}-\left\|k_{n}-p\right\|^{2} \\
&+2 \lambda_{i, n}\left\|\Omega_{n}^{i-1} u_{n}-\Omega_{n}^{i} u_{n}\right\|\left\|A_{i} \Omega_{n}^{i-1} u_{n}-A_{i} p\right\| \\
&+2 \lambda_{n} \alpha_{n}\|p\|\left\|p-t_{n}\right\|+\gamma_{n}\left\|z_{n}-p\right\|^{2}+c_{n} \\
& \leq\left\|x_{n}-k_{n}\right\|\left(\left\|x_{n}-p\right\|+\left\|k_{n}-p\right\|\right) \\
&+2 \lambda_{i, n}\left\|\Omega_{n}^{i-1} u_{n}-\Omega_{n}^{i} u_{n}\right\|\left\|A_{i} \Omega_{n}^{i-1} u_{n}-A_{i} p\right\| \\
&+2 \lambda_{n} \alpha_{n}\|p\|\left\|p-t_{n}\right\|+\gamma_{n}\left\|z_{n}-p\right\|^{2}+c_{n} .
\end{aligned}
$$

Since $\left\{\lambda_{n}\right\} \subset[c, d] \subset(0,1 / L),\left\{\lambda_{i, n}\right\} \subset\left[a_{i}, b_{i}\right] \subset\left(0,2 \eta_{i}\right)$, $i \in\{1,2, \ldots, N\}, \alpha_{n} \rightarrow 0, \gamma_{n} \rightarrow 0$, and $c_{n} \rightarrow 0$, we conclude from (152) and (163) and the boundedness of $\left\{x_{n}\right\},\left\{k_{n}\right\},\left\{u_{n}\right\},\left\{t_{n}\right\}$, and $\left\{z_{n}\right\}$ that

$$
\lim _{n \rightarrow \infty}\left\|\Omega_{n}^{i-1} u_{n}-\Omega_{n}^{i} u_{n}\right\|=0, \quad i \in\{1,2, \ldots, N\} .
$$

By (166), we have

$$
\begin{aligned}
\left\|u_{n}-\tilde{x}_{n}\right\|= & \left\|\Omega_{n}^{0} u_{n}-\Omega_{n}^{N} u_{n}\right\| \\
\leq & \left\|\Omega_{n}^{0} u_{n}-\Omega_{n}^{1} u_{n}\right\|+\left\|\Omega_{n}^{1} u_{n}-\Omega_{n}^{2} u_{n}\right\| \\
& +\cdots+\left\|\Omega_{n}^{N-1} u_{n}-\Omega_{n}^{N} u_{n}\right\| \\
& \longrightarrow 0 \text { as } n \longrightarrow \infty .
\end{aligned}
$$

From (160) and (167), we have

$$
\left\|x_{n}-\tilde{x}_{n}\right\| \leq\left\|x_{n}-u_{n}\right\|+\left\|u_{n}-\tilde{x}_{n}\right\| \longrightarrow 0 \quad \text { as } n \longrightarrow \infty \text {. }
$$

By (152) and (168), we obtain

$$
\left\|k_{n}-\tilde{x}_{n}\right\| \leq\left\|k_{n}-x_{n}\right\|+\left\|x_{n}-\tilde{x}_{n}\right\| \longrightarrow 0 \text { as } n \longrightarrow \infty .
$$
have

Furthermore, combining (139), (140), (142), and (147), we

$$
\begin{aligned}
\left\|k_{n}-p\right\|^{2} \leq & \left\|z_{n}-p\right\|^{2}+\gamma_{n}\left\|z_{n}-p\right\|^{2}+c_{n} \\
\leq & \left\|\tilde{x}_{n}-p\right\|^{2}+2 \lambda_{n} \alpha_{n}\|p\|\left\|p-t_{n}\right\| \\
& +\left(\lambda_{n}^{2}\left(\alpha_{n}+L\right)^{2}-1\right)\left\|\tilde{x}_{n}-t_{n}\right\|^{2} \\
& +\gamma_{n}\left\|z_{n}-p\right\|^{2}+c_{n} \\
\leq & \left\|x_{n}-p\right\|^{2}+2 \lambda_{n} \alpha_{n}\|p\|\left\|p-t_{n}\right\| \\
& +\left(\lambda_{n}^{2}\left(\alpha_{n}+L\right)^{2}-1\right)\left\|\tilde{x}_{n}-t_{n}\right\|^{2} \\
& +\gamma_{n}\left\|z_{n}-p\right\|^{2}+c_{n},
\end{aligned}
$$


which yields

$$
\begin{aligned}
(1- & \left.\lambda_{n}^{2}\left(\alpha_{n}+L\right)^{2}\right)\left\|\tilde{x}_{n}-t_{n}\right\|^{2} \\
\leq & \left\|x_{n}-p\right\|^{2}-\left\|k_{n}-p\right\|^{2}+2 \lambda_{n} \alpha_{n}\|p\|\left\|p-t_{n}\right\| \\
& +\gamma_{n}\left\|z_{n}-p\right\|^{2}+c_{n} \\
\leq & \left\|x_{n}-k_{n}\right\|\left(\left\|x_{n}-p\right\|+\left\|k_{n}-p\right\|\right) \\
& +2 \lambda_{n} \alpha_{n}\|p\|\left\|p-t_{n}\right\|+\gamma_{n}\left\|z_{n}-p\right\|^{2}+c_{n} .
\end{aligned}
$$

Since $\left\{\lambda_{n}\right\} \subset[c, d] \subset(0,1 / L), \alpha_{n} \rightarrow 0, \gamma_{n} \rightarrow 0$, and $c_{n} \rightarrow 0$, we obtain from (152) and the boundedness of $\left\{x_{n}\right\},\left\{k_{n}\right\}\left\{t_{n}\right\}$, and $\left\{z_{n}\right\}$ that

$$
\lim _{n \rightarrow \infty}\left\|\tilde{x}_{n}-t_{n}\right\|=0
$$

Also, combining (139), (140), (4.5)' , and (147), we have

$$
\begin{aligned}
\left\|k_{n}-p\right\|^{2} \leq & \left\|z_{n}-p\right\|^{2}+\gamma_{n}\left\|z_{n}-p\right\|^{2}+c_{n} \\
\leq & \left\|\tilde{x}_{n}-p\right\|^{2}+2 \lambda_{n} \alpha_{n}\|p\|\left\|p-t_{n}\right\| \\
& +\left(\lambda_{n}^{2}\left(\alpha_{n}+L\right)^{2}-1\right)\left\|\tilde{x}_{n}-t_{n}\right\|^{2} \\
& +\gamma_{n}\left\|z_{n}-p\right\|^{2}+c_{n} \\
\leq & \left\|x_{n}-p\right\|^{2}+2 \lambda_{n} \alpha_{n}\|p\|\left\|p-t_{n}\right\| \\
& +\left(\lambda_{n}^{2}\left(\alpha_{n}+L\right)^{2}-1\right)\left\|t_{n}-z_{n}\right\|^{2} \\
& +\gamma_{n}\left\|z_{n}-p\right\|^{2}+c_{n},
\end{aligned}
$$

which leads to

$$
\begin{aligned}
(1- & \left.\lambda_{n}^{2}\left(\alpha_{n}+L\right)^{2}\right)\left\|t_{n}-z_{n}\right\|^{2} \\
\leq & \left\|x_{n}-p\right\|^{2}-\left\|k_{n}-p\right\|^{2} \\
& +2 \lambda_{n} \alpha_{n}\|p\|\left\|p-t_{n}\right\|+\gamma_{n}\left\|z_{n}-p\right\|^{2}+c_{n} \\
\leq & \left\|x_{n}-k_{n}\right\|\left(\left\|x_{n}-p\right\|+\left\|k_{n}-p\right\|\right) \\
& +2 \lambda_{n} \alpha_{n}\|p\|\left\|p-t_{n}\right\|+\gamma_{n}\left\|z_{n}-p\right\|^{2}+c_{n} .
\end{aligned}
$$

Since $\left\{\lambda_{n}\right\} \subset[c, d] \subset(0,1 / L), \alpha_{n} \rightarrow 0, \gamma_{n} \rightarrow 0$, and $c_{n} \rightarrow 0$, we obtain from (152) and the boundedness of $\left\{x_{n}\right\},\left\{k_{n}\right\},\left\{t_{n}\right\}$, and $\left\{z_{n}\right\}$ that

$$
\lim _{n \rightarrow \infty}\left\|t_{n}-z_{n}\right\|=0
$$

Hence, combining (172) and (175), we get

$$
\left\|\tilde{x}_{n}-z_{n}\right\| \leq\left\|\tilde{x}_{n}-t_{n}\right\|+\left\|t_{n}-z_{n}\right\| \longrightarrow 0 \text { as } n \longrightarrow \infty \text {. }
$$

We note that

$$
k_{n}-\tilde{x}_{n}=\left(1-\delta_{n}\right)\left(S^{n} z_{n}-z_{n}\right) .
$$

From $\delta_{n} \leq b<1$ and (169), we have

$$
\lim _{n \rightarrow \infty}\left\|S^{n} z_{n}-z_{n}\right\|=0
$$

On the other hand, we observe that

$$
\begin{aligned}
\left\|z_{n+1}-z_{n}\right\| \leq & \left\|z_{n+1}-\tilde{x}_{n+1}\right\|+\left\|\tilde{x}_{n+1}-x_{n+1}\right\| \\
& +\left\|x_{n+1}-x_{n}\right\|+\left\|x_{n}-\tilde{x}_{n}\right\|+\left\|\tilde{x}_{n}-z_{n}\right\| .
\end{aligned}
$$

By (153), (168), and (176), we have

$$
\lim _{n \rightarrow \infty}\left\|z_{n+1}-z_{n}\right\|=0
$$

We note that

$$
\begin{aligned}
\| z_{n}- & S z_{n}\|\leq\| z_{n}-z_{n+1}\|+\| z_{n+1}-S^{n+1} z_{n+1} \| \\
& +\left\|S^{n+1} z_{n+1}-S^{n+1} z_{n}\right\|+\left\|S^{n+1} z_{n}-S z_{n}\right\| .
\end{aligned}
$$

From (178), (180), Lemma 14, and the uniform continuity of $S$, we obtain

$$
\lim _{n \rightarrow \infty}\left\|z_{n}-S z_{n}\right\|=0
$$

Since $\left\{x_{n}\right\}$ is bounded, there exists a subsequence $\left\{x_{n_{i}}\right\}$ of $\left\{x_{n}\right\}$ which converges weakly to $w$. From (168) and (176), we have that $z_{n_{i}} \rightarrow w$. From (182) and the uniform continuity of $S$, we have $\lim _{n \rightarrow \infty}\left\|z_{n}-S^{m} z_{n}\right\|=0$ for any $m \geq 1$. So, from Lemma 16, we have $w \in \operatorname{Fix}(S)$. Similarly to the arguments in the proof of Theorem 24, we can derive $w \in$ $\cap_{k=1}^{M} \operatorname{GMEP}\left(F_{k}, \varphi_{k}, B_{k}\right) \cap \cap_{i=1}^{N} \operatorname{VI}\left(C, A_{i}\right) \cap \Gamma$. Consequently, $w \in F$. This shows that $\omega_{w}\left(x_{n}\right) \subset F$.

Next let us show that $\omega_{w}\left(x_{n}\right)$ is a single-point set. As a matter of fact, let $\left\{x_{n_{j}}\right\}$ be another subsequence of $\left\{x_{n}\right\}$ such that $x_{n_{j}} \rightarrow w^{\prime}$. Then we get $w^{\prime} \in F$. If $w \neq w^{\prime}$, from the Opial condition, we have

$$
\begin{aligned}
\lim _{n \rightarrow \infty}\left\|x_{n}-w\right\| & =\lim _{i \rightarrow \infty}\left\|x_{n_{i}}-w\right\|<\lim _{i \rightarrow \infty}\left\|x_{n_{i}}-w^{\prime}\right\| \\
& =\lim _{n \rightarrow \infty}\left\|x_{n}-w^{\prime}\right\|=\lim _{j \rightarrow \infty}\left\|x_{n_{j}}-w^{\prime}\right\| \\
& <\lim _{j \rightarrow \infty}\left\|x_{n_{j}}-w\right\|=\lim _{n \rightarrow \infty}\left\|x_{n}-w\right\| .
\end{aligned}
$$

This attains a contraction. So we have $w=w^{\prime}$. This shows that $\omega_{w}\left(x_{n}\right)=\{w\}$. Therefore, by Lemma 10, we know that $x_{n} \rightarrow w$.

Finally, we claim that $w=\lim _{n \rightarrow \infty} P_{F} x_{n}$ provided $F$ is bounded. Put $v_{n}=P_{F}\left(x_{n}\right)$. Since $w \in F$, we have $\left\langle x_{n}-v_{n}, v_{n}-\right.$ $w\rangle \geq 0$. By (148) and Lemma 21 , we have $\left\{v_{n}\right\}$ which converges strongly to some $w_{0} \in F$. Since $\left\{x_{n}\right\}$ converges weakly to $w$, we have

$$
\left\langle w-w_{0}, w_{0}-w\right\rangle \geq 0
$$

Therefore, we obtain $w=w_{0}=\lim _{n \rightarrow \infty} P_{F} x_{n}$. This completes the proof. 
Corollary 31 (i.e., [3, Theorem 4.1]). Let $C$ be a nonempty closed convex subset of a real Hilbert space $H$. Let $M, N$ be two integers. Let $F_{k}$ be a bifunction from $C \times C$ to $\mathbf{R}$ satisfying (A1)-(A4) and let $\varphi_{k}: C \rightarrow \mathbf{R} \cup\{+\infty\}$ be a proper lower semicontinuous and convex function, where $k \in\{1,2, \ldots, M\}$. Let $B_{k}$ and $A_{i}$ be $\mu_{k}$-inverse strongly monotone and $\eta_{i}$-inversestrongly monotone, respectively, where $k \in\{1,2, \ldots, M\}$, $i \in\{1,2, \ldots, N\}$. Let $S: C \rightarrow C$ be the uniformly continuous asymptotically $\kappa$-strict pseudocontractive mapping in the intermediate sense for some $0 \leq \kappa<1$ with sequences $\left\{\gamma_{n}\right\} \subset[0, \infty)$ and $\left\{c_{n}\right\} \subset[0, \infty)$. Assume that $F:=$ $\cap_{k=1}^{M} \operatorname{GMEP}\left(F_{k}, \varphi_{k}, A_{k}\right) \cap \cap_{i=1}^{N} \operatorname{VI}\left(C, A_{i}\right) \cap \operatorname{Fix}(S)$ is nonempty. Let $\left\{\beta_{n}\right\}$ and $\left\{\delta_{n}\right\}$ be sequences in $[0,1]$ such that $0<a \leq \beta_{n} \leq$ $\beta<1$ and $\kappa \leq \delta_{n} \leq b<1$. Pick any $x_{1} \in H$ and let $\left\{x_{n}\right\}$ be $a$ sequence generated by the following algorithm:

$$
\begin{gathered}
u_{n}=T_{r_{M, n}}^{\left(F_{M}, \varphi_{M}\right)}\left(I-r_{M, n} B_{M}\right) T_{r_{M, n}}^{\left(F_{M-1}, \varphi_{M-1}\right)} \\
\times\left(I-r_{M-1, n} B_{M-1}\right) \cdots T_{r_{1, n}}^{\left(F_{1}, \varphi_{1}\right)}\left(I-r_{1, n} B_{1}\right) x_{n}, \\
z_{n}=P_{C}\left(I-\lambda_{N, n} A_{N}\right) P_{C}\left(I-\lambda_{N-1, n} A_{N-1}\right) \\
\cdots P_{C}\left(I-\lambda_{2, n} A_{2}\right) P_{C}\left(I-\lambda_{1, n} A_{1}\right) u_{n}, \\
k_{n}=\delta_{n} z_{n}+\left(1-\delta_{n}\right) S^{n} z_{n}, \\
x_{n+1}=\left(1-\beta_{n}\right) x_{n}+\beta_{n} k_{n},
\end{gathered}
$$

where $\left\{\lambda_{i, n}\right\} \subset\left[a_{i}, b_{i}\right] \subset\left(0,2 \eta_{i}\right),\left\{r_{k, n}\right\} \subset\left[e_{k}, f_{k}\right] \subset\left(0,2 \mu_{k}\right), i \in$ $\{1,2, \ldots, N\}$, and $k \in\{1,2, \ldots, M\}$. Assume that $\sum_{n=1}^{\infty} \gamma_{n}<\infty$ and $\sum_{n=1}^{\infty} c_{n}<\infty$, and that either (B1) or (B2) holds. Then $\left\{x_{n}\right\}$ converges weakly to $w=\lim _{n \rightarrow \infty} P_{F} x_{n}$.

Proof. In Theorem 30, put $\nabla f=0$ and $\alpha_{n}=0$ for all $n \geq 1$. Then $\Gamma=C$ and $F=\cap_{k=1}^{M} \operatorname{GMEP}\left(F_{k}, \varphi_{k}, A_{k}\right) \cap$ $\cap_{i=1}^{N} \operatorname{VI}\left(C, A_{i}\right) \cap \operatorname{Fix}(S) \cap \Gamma=\cap_{k=1}^{M} \operatorname{GMEP}\left(F_{k}, \varphi_{k}, A_{k}\right) \cap$ $\cap_{i=1}^{N} \operatorname{VI}\left(C, A_{i}\right) \cap \operatorname{Fix}(S)$. In this case, we obtain from (134) that

$$
\begin{aligned}
& t_{n}=P_{C}\left(\tilde{x}_{n}-\lambda_{n} \nabla f_{\alpha_{n}}\left(\tilde{x}_{n}\right)\right)=\tilde{x}_{n}, \\
& z_{n}=P_{C}\left(\tilde{x}_{n}-\lambda_{n} \nabla f_{\alpha_{n}}\left(t_{n}\right)\right)=\tilde{x}_{n} .
\end{aligned}
$$

Thus, the iterative scheme (134) reduces to (95). It is easy to see that all the conditions of Theorem 24 are satisfied. In terms of Theorem 24 , we have that $\left\{x_{n}\right\}$ converges weakly to an element $w \in F$. Now, put $v_{n}=P_{F}\left(x_{n}\right)$. Since $w \in F$, we have $\left\langle x_{n}-v_{n}, v_{n}-w\right\rangle \geq 0$. Taking into account that $\alpha_{n}=0$ for all $n \geq 1$, we conclude from (148) that

$$
\begin{aligned}
\left\|x_{n+1}-p\right\|^{2} \leq & {\left[1+\gamma_{n}+\left(1+\gamma_{n}\right) \lambda_{n} \alpha_{n}\right]\left\|x_{n}-p\right\|^{2} } \\
& +3\left(1+\gamma_{n}\right) \lambda_{n} \alpha_{n}\|p\|^{2}+c_{n} \\
\leq & \left(1+\gamma_{n}\right)\left\|x_{n}-p\right\|^{2}+c_{n} .
\end{aligned}
$$

By Lemma 21, we have that $\left\{v_{n}\right\}$ converges strongly to some $w_{0} \in F$. Since $\left\{x_{n}\right\}$ converges weakly to $w$, we have

$$
\left\langle w-w_{0}, w_{0}-w\right\rangle \geq 0 .
$$

Therefore, we obtain $w=w_{0}=\lim _{n \rightarrow \infty} P_{F} x_{n}$. This completes the proof.
Corollary 32. Let $C$ be a nonempty closed convex subset of a real Hilbert space $H$. Let $f: C \rightarrow \mathbf{R}$ be a convex functional with L-Lipschitz continuous gradient $\nabla f$. Let $F$ be a bifunction from $C \times C$ to $\mathbf{R}$ satisfying (A1)-(A4) and let $\varphi$ : $C \rightarrow \mathbf{R} \cup\{+\infty\}$ be a proper lower semicontinuous and convex function. Let $B$ and $A_{i}$ be $\mu$-inverse strongly monotone and $\eta_{i^{-}}$ inverse-strongly monotone, respectively, where $i \in\{1,2\}$. Let $S: C \rightarrow C$ be the uniformly continuous asymptotically $\kappa$-strict pseudocontractive mapping in the intermediate sense for some $0 \leq \kappa<1$ with sequences $\left\{\gamma_{n}\right\} \subset[0, \infty)$ and $\left\{c_{n}\right\} \subset[0, \infty)$. Assume that $F:=\operatorname{GMEP}(F, \varphi, B) \cap \operatorname{VI}\left(C, A_{1}\right) \cap \operatorname{VI}\left(C, A_{2}\right) \cap$ $\operatorname{Fix}(S) \cap \Gamma$ is nonempty. Let $\left\{\alpha_{n}\right\}$ is a sequence in $(0, \infty)$, and let $\left\{\beta_{n}\right\},\left\{\delta_{n}\right\}$ be sequences in $[0,1]$ such that $0<a \leq \beta_{n} \leq \beta<1$ and $\kappa \leq \delta_{n} \leq b<1$. Pick any $x_{1} \in H$ and let $\left\{x_{n}\right\}$ be a sequence generated by the following algorithm:

$$
\begin{gathered}
F\left(u_{n}, y\right)+\varphi(y)-\varphi\left(u_{n}\right)+\left\langle B x_{n}, y-u_{n}\right\rangle \\
+\frac{1}{r_{n}}\left\langle y-u_{n}, u_{n}-x_{n}\right\rangle \geq 0, \quad \forall y \in C, \\
\tilde{x}_{n}=P_{C}\left(I-\lambda_{2, n} A_{2}\right) P_{C}\left(I-\lambda_{1, n} A_{1}\right) u_{n}, \\
t_{n}=P_{C}\left(\tilde{x}_{n}-\lambda_{n} \nabla f_{\alpha_{n}}\left(\tilde{x}_{n}\right)\right), \\
z_{n}=P_{C}\left(\tilde{x}_{n}-\lambda_{n} \nabla f_{\alpha_{n}}\left(t_{n}\right)\right), \\
k_{n}=\delta_{n} z_{n}+\left(1-\delta_{n}\right) S^{n} z_{n}, \\
x_{n+1}=\left(1-\beta_{n}\right) x_{n}+\beta_{n} k_{n},
\end{gathered}
$$

where $\left\{\lambda_{i, n}\right\} \subset\left[a_{i}, b_{i}\right] \subset\left(0,2 \eta_{i}\right),\left\{r_{n}\right\} \subset[e, f] \subset(0,2 \mu)$, and $i \in\{1,2\}$. Assume that either (B1) or (B2) holds and that the following conditions are satisfied:

(i) $\sum_{n=1}^{\infty} \alpha_{n}<\infty, \sum_{n=1}^{\infty} \gamma_{n}<\infty$ and $\sum_{n=1}^{\infty} c_{n}<\infty$;

(ii) $\left\{\lambda_{n}\right\} \subset[c, d]$ for some $c, d \in(0,1 / L)$.

Then

(a) $\left\{x_{n}\right\}$ converges weakly to an element $w \in F$;

(b) $\left\{x_{n}\right\}$ converges weakly to $w=\lim _{n \rightarrow \infty} P_{F} x_{n}$ provided $F$ is bounded.

Corollary 33. Let $C$ be a nonempty closed convex subset of a real Hilbert space $H$. Let $f: C \rightarrow \mathbf{R}$ be a convex functional with L-Lipschitz continuous gradient $\nabla f$. Let $F$ be a bifunction from $C \times C$ to $\mathbf{R}$ satisfying (A1)-(A4) and let $\varphi: C \rightarrow \mathbf{R} \cup\{+\infty\}$ be a proper lower semicontinuous and convex function. Let $B$ and $A$ be $\mu$-inverse strongly monotone and $\eta$-inverse-strongly monotone, respectively. Let $S: C \rightarrow C$ be the uniformly continuous asymptotically $\kappa$-strict pseudocontractive mapping in the intermediate sense for some $0 \leq \kappa<1$ with sequences $\left\{\gamma_{n}\right\} \subset[0, \infty)$ and $\left\{c_{n}\right\} \subset[0, \infty)$. Assume that $F:=\operatorname{GMEP}(F, \varphi, B) \cap \operatorname{VI}(C, A) \cap \operatorname{Fix}(S) \cap \Gamma$ is nonempty. Let $\left\{\alpha_{n}\right\}$ be a sequence in $(0, \infty)$, and let $\left\{\beta_{n}\right\},\left\{\delta_{n}\right\}$ be sequences in $[0,1]$ such that $0<a \leq \beta_{n} \leq \beta<1$ and 
$\kappa \leq \delta_{n} \leq b<1$. Pick any $x_{1} \in H$ and let $\left\{x_{n}\right\}$ be a sequence generated by the following algorithm:

$$
\begin{gathered}
F\left(u_{n}, y\right)+\varphi(y)-\varphi\left(u_{n}\right)+\left\langle B x_{n}, y-u_{n}\right\rangle \\
+\frac{1}{r_{n}}\left\langle y-u_{n}, u_{n}-x_{n}\right\rangle \geq 0, \quad \forall y \in C, \\
\tilde{x}_{n}=P_{C}\left(u_{n}-\rho_{n} A u_{n}\right), \\
t_{n}=P_{C}\left(\tilde{x}_{n}-\lambda_{n} \nabla f_{\alpha_{n}}\left(\tilde{x}_{n}\right)\right), \\
z_{n}=P_{C}\left(\tilde{x}_{n}-\lambda_{n} \nabla f_{\alpha_{n}}\left(t_{n}\right)\right), \\
k_{n}=\delta_{n} z_{n}+\left(1-\delta_{n}\right) S^{n} z_{n}, \\
x_{n+1}=\left(1-\beta_{n}\right) x_{n}+\beta_{n} k_{n},
\end{gathered}
$$

where $\left\{\rho_{n}\right\} \subset[a, b] \subset(0,2 \eta),\left\{r_{n}\right\} \subset[e, f] \subset(0,2 \mu)$. Assume that either (B1) or (B2) holds and that the following conditions are satisfied:

(i) $\sum_{n=1}^{\infty} \alpha_{n}<\infty, \sum_{n=1}^{\infty} \gamma_{n}<\infty$ and $\sum_{n=1}^{\infty} c_{n}<\infty$;

(ii) $\left\{\lambda_{n}\right\} \subset[c, d]$ for some $c, d \in(0,1 / L)$.

Then

(a) $\left\{x_{n}\right\}$ converges weakly to an element $w \in F$;

(b) $\left\{x_{n}\right\}$ converges weakly to $w=\lim _{n \rightarrow \infty} P_{F} x_{n}$ provided $F$ is bounded.

Corollary 34. Let $C$ be a nonempty closed convex subset of a real Hilbert space $H$. Let $f: C \rightarrow \mathbf{R}$ be a convex functional with L-Lipschitz continuous gradient $\nabla f$. Let $F$ be a bifunction from $C \times C$ to $\mathbf{R}$ satisfying (A1)-(A4) and let $\varphi: C \rightarrow \mathbf{R} \cup\{+\infty\}$ be a proper lower semicontinuous and convex function. Let $B$ and $A$ be $\mu$-inverse strongly monotone and $\eta$-inverse-strongly monotone, respectively. Let $S: C \rightarrow C$ be the uniformly continuous asymptotically $\kappa$-strict pseudocontractive mapping for some $0 \leq \kappa<1$ with sequence $\left\{\gamma_{n}\right\} \subset[0, \infty)$. Assume that $F:=\operatorname{GMEP}(F, \varphi, B) \cap \operatorname{VI}(C, A) \cap$ $\operatorname{Fix}(S) \cap \Gamma$ is nonempty. Let $\left\{\alpha_{n}\right\}$ be a sequence in $(0, \infty)$, and let $\left\{\beta_{n}\right\},\left\{\delta_{n}\right\}$ be sequences in $[0,1]$ such that $0<a \leq \beta_{n} \leq \beta<1$ and $\kappa \leq \delta_{n} \leq b<1$. Pick any $x_{1} \in H$ and let $\left\{x_{n}\right\}$ be a sequence generated by the following algorithm:

$$
\begin{gathered}
F\left(u_{n}, y\right)+\varphi(y)-\varphi\left(u_{n}\right)+\left\langle B x_{n}, y-u_{n}\right\rangle \\
+\frac{1}{r_{n}}\left\langle y-u_{n}, u_{n}-x_{n}\right\rangle \geq 0, \quad \forall y \in C, \\
\tilde{x}_{n}=P_{C}\left(u_{n}-\rho_{n} A u_{n}\right), \\
t_{n}=P_{C}\left(\tilde{x}_{n}-\lambda_{n} \nabla f_{\alpha_{n}}\left(\tilde{x}_{n}\right)\right), \\
z_{n}=P_{C}\left(\tilde{x}_{n}-\lambda_{n} \nabla f_{\alpha_{n}}\left(t_{n}\right)\right), \\
k_{n}=\delta_{n} z_{n}+\left(1-\delta_{n}\right) S^{n} z_{n}, \\
x_{n+1}=\left(1-\beta_{n}\right) x_{n}+\beta_{n} k_{n},
\end{gathered}
$$

where $\left\{\rho_{n}\right\} \subset[a, b] \subset(0,2 \eta),\left\{r_{n}\right\} \subset[e, f] \subset(0,2 \mu)$. Assume that either (B1) or (B2) holds and that the following conditions are satisfied:

(i) $\sum_{n=1}^{\infty} \alpha_{n}<\infty$ and $\sum_{n=1}^{\infty} \gamma_{n}<\infty$;

(ii) $\left\{\lambda_{n}\right\} \subset[c, d]$ for some $c, d \in(0,1 / L)$.

Then

(a) $\left\{x_{n}\right\}$ converges weakly to an element $w \in F$;

(b) $\left\{x_{n}\right\}$ converges weakly to $w=\lim _{n \rightarrow \infty} P_{F} x_{n}$ provided $F$ is bounded.

Finally, we provide an example to illustrate Corollary 34.

Example 35. Let $H=\mathbf{R}^{2}$ with inner product $\langle\cdot, \cdot\rangle$ and norm $\|\cdot\|$ which are defined by

$$
\langle x, y\rangle=a_{1} b_{1}+a_{2} b_{2}, \quad\|x\|=\sqrt{a_{1}^{2}+a_{2}^{2}}
$$

for all $x, y \in \mathbf{R}^{2}$ with $x=\left(a_{1}, a_{2}\right)$ and $y=\left(b_{1}, b_{2}\right)$. Let $C=\{(a, a): a \in \mathbf{R}\}$. Clearly, $C$ is a nonempty closed convex subset of a real Hilbert space $H=\mathbf{R}^{2}$. Let $f: C \rightarrow \mathbf{R}$ be a convex functional with $L$-Lipschitz continuous gradient $\nabla f$, for instance, putting $f(x)=(1 / 2)\langle V x, x\rangle$, where $V=$ $\left\{\begin{array}{ll}3 / 5 & 2 / 5 \\ 2 / 5 & 3 / 5\end{array}\right\}$. Then $\nabla f=V$ is $L$-Lipschitz continuous with $L=1$ (due to $\|V\|=1$ ). Put $F(x, y)=0$ and $\varphi(x)=0$ for all $x, y \in C$. Then it is clear that $F$ is a bifunction from $C \times C$ to $R$ satisfying (A1)-(A4) and let $\varphi: C \rightarrow \mathbf{R} \cup\{+\infty\}$ be a proper lower semicontinuous and convex function. Let $B$ and $A$ be $\mu$-inverse strongly monotone and $\eta$-inverse-strongly monotone, respectively, for instance, putting $A=\left\{\begin{array}{lll}2 / 3 & 1 / 3 \\ 1 / 3 & 2 / 3\end{array}\right\}$ and $B=\left\{\begin{array}{ll}3 / 5 & 2 / 5 \\ 2 / 5 & 3 / 5\end{array}\right\}$. Then we can take $\mu=\eta=1 / 2$. Let $S: C \rightarrow C$ be the uniformly continuous asymptotically $\kappa$ strict pseudocontractive mapping for some $0 \leq \kappa<1$ with

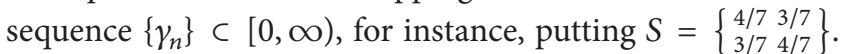
Then $\kappa=0$ and $\gamma_{n}=0$ for all $n \geq 1$ (due to the nonexpansivity of $S)$. Thus, we know that $F:=\operatorname{GMEP}(F, \varphi, B) \cap \operatorname{VI}(C, A) \cap$ $\operatorname{Fix}(S) \cap \Gamma=\operatorname{GMEP}(0,0, B) \cap \operatorname{VI}(C, A) \cap \operatorname{Fix}(S) \cap \Gamma=\{0\}$. Take $\left\{\rho_{n}\right\} \subset[a, b] \subset(0,2 \eta)=(0,1),\left\{r_{n}\right\} \subset[e, f] \subset(0,2 \mu)=(0,1)$, and $\left\{\lambda_{n}\right\} \subset[c, d] \subset(0,1 / L)=(0,1)$. Let $\left\{\alpha_{n}\right\}$ be a sequence in $(0, \infty)$ such that $\lim _{n \rightarrow \infty} \alpha_{n}=0$, and let $\left\{\beta_{n}\right\},\left\{\delta_{n}\right\}$ be sequences in $[0,1]$ such that $0<a \leq \beta_{n} \leq \beta<1$ and $0=\kappa \leq \delta_{n} \leq b<1$. Pick any $x_{1} \in C$. In this case, the algorithm (191) reduces to the following algorithm:

$$
\begin{gathered}
u_{n}=P_{C}\left(x_{n}-r_{n} B x_{n}\right)=\left(1-r_{n}\right) x_{n}, \\
\tilde{x}_{n}=P_{C}\left(u_{n}-\rho_{n} A u_{n}\right)=\left(1-\rho_{n}\right)\left(1-r_{n}\right) x_{n} \\
t_{n}=P_{C}\left(\tilde{x}_{n}-\lambda_{n} \nabla f_{\alpha_{n}}\left(\tilde{x}_{n}\right)\right) \\
=\left(1-\lambda_{n}\left(1+\alpha_{n}\right)\right)\left(1-\rho_{n}\right)\left(1-r_{n}\right) x_{n},
\end{gathered}
$$




$$
\begin{gathered}
z_{n}=P_{C}\left(\tilde{x}_{n}-\lambda_{n} \nabla f_{\alpha_{n}}\left(t_{n}\right)\right)=\left(1-\rho_{n}\right)\left(1-r_{n}\right) x_{n} \\
-\lambda_{n}\left(1+\alpha_{n}\right)\left(1-\lambda_{n}\left(1+\alpha_{n}\right)\right)\left(1-\rho_{n}\right)\left(1-r_{n}\right) x_{n} \\
=\left[1-\lambda_{n}\left(1+\alpha_{n}\right)\left(1-\lambda_{n}\left(1+\alpha_{n}\right)\right)\right]\left(1-\rho_{n}\right)\left(1-r_{n}\right) x_{n}, \\
\quad k_{n}=\delta_{n} z_{n}+\left(1-\delta_{n}\right) S^{n} z_{n}=z_{n}, \\
x_{n+1}=\left(1-\beta_{n}\right) x_{n}+\beta_{n} k_{n} \\
=\left\{\left(1-\beta_{n}\right)+\beta_{n}\left[1-\lambda_{n}\left(1+\alpha_{n}\right)\left(1-\lambda_{n}\left(1+\alpha_{n}\right)\right)\right]\right. \\
\left.\quad \times\left(1-\rho_{n}\right)\left(1-r_{n}\right)\right\} x_{n} .
\end{gathered}
$$

So it follows that

$$
\begin{aligned}
&\left\|x_{n+1}\right\| \\
&=\left\{\left(1-\beta_{n}\right)+\beta_{n}\left[1-\lambda_{n}\left(1+\alpha_{n}\right)\left(1-\lambda_{n}\left(1+\alpha_{n}\right)\right)\right]\right. \\
&\left.\quad \times\left(1-\rho_{n}\right)\left(1-r_{n}\right)\right\}\left\|x_{n}\right\| \\
& \leq\left\{\left(1-\beta_{n}\right)+\beta_{n}\left[1-\lambda_{n}\left(1+\alpha_{n}\right)\left(1-\lambda_{n}\left(1+\alpha_{n}\right)\right)\right]\right\}\left\|x_{n}\right\| \\
&=\left\{1-\beta_{n} \lambda_{n}\left(1+\alpha_{n}\right)\left(1-\lambda_{n}\left(1+\alpha_{n}\right)\right)\right\}\left\|x_{n}\right\| \\
& \leq {\left[1-a c\left(1+\alpha_{n}\right)\left(1-d\left(1+\alpha_{n}\right)\right)\right]\left\|x_{n}\right\| . }
\end{aligned}
$$

Since $\lim _{n \rightarrow \infty} a c\left(1+\alpha_{n}\right)\left(1-d\left(1+\alpha_{n}\right)\right)=a c(1-d)>$ $(1 / 2) a c(1-d)$, we deduce that there exists an integer $n_{0} \geq 1$ such that

$$
a c\left(1+\alpha_{n}\right)\left(1-d\left(1+\alpha_{n}\right)\right)>\frac{1}{2} a c(1-d), \quad \forall n \geq n_{0} .
$$

Therefore, from (194) we obtain that for all $n \geq n_{0}$

$$
\begin{aligned}
\left\|x_{n+1}\right\| & \leq\left[1-a c\left(1+\alpha_{n}\right)\left(1-d\left(1+\alpha_{n}\right)\right)\right]\left\|x_{n}\right\| \\
& \leq\left[1-\frac{1}{2} a c(1-d)\right]\left\|x_{n}\right\| \\
& \vdots \\
& \leq\left[1-\frac{1}{2} a c(1-d)\right]^{n-n_{0}+1}\left\|x_{n_{0}}\right\| .
\end{aligned}
$$

This shows that

(a) $\left\{x_{n}\right\}$ converges to the unique point 0 in $F$;

(b) $\left\{x_{n}\right\}$ converges to $0=\lim _{n \rightarrow \infty} P_{F} x_{n}$.

\section{Conflict of Interests}

The authors declare that there is no conflict of interests regarding the publication of this paper.

\section{Acknowledgments}

This research was partially supported by the National Science Foundation of China (11071169), Innovation Program of Shanghai Municipal Education Commission (09ZZ133), and Leading Academic Discipline Project of Shanghai Normal University (DZL707). This research was partially supported by a grant from the NSC (101-2115-M-165 -001) as well.

\section{References}

[1] J.-W. Peng and J.-C. Yao, "A new hybrid-extragradient method for generalized mixed equilibrium problems, fixed point problems and variational inequality problems," Taiwanese Journal of Mathematics, vol. 12, no. 6, pp. 1401-1432, 2008.

[2] L. C. Ceng, H.-Y. Hu, and M. M. Wong, "Strong and weak convergence theorems for generalized mixed equilibrium problem with perturbation and fixed pointed problem of infinitely many nonexpansive mappings," Taiwanese Journal of Mathematics, vol. 15, no. 3, pp. 1341-1367, 2011.

[3] G. Cai and S. Bu, "Strong and weak convergence theorems for general mixed equilibrium problems and variational inequality problems and fixed point problems in Hilbert spaces," Journal of Computational and Applied Mathematics, vol. 247, pp. 34-52, 2013.

[4] L.-C. Ceng and J.-C. Yao, "A relaxed extragradient-like method for a generalized mixed equilibrium problem, a general system of generalized equilibria and a fixed point problem," Nonlinear Analysis: Theory, Methods \& Applications, vol. 72, no. 3-4, pp. 1922-1937, 2010.

[5] L.-C. Ceng, Q. H. Ansari, and S. Schaible, "Hybrid extragradient-like methods for generalized mixed equilibrium problems, systems of generalized equilibrium problems and optimization problems," Journal of Global Optimization, vol. 53, no. 1, pp. 69-96, 2012.

[6] J.-B. Baillon and G. Haddad, "Quelques propriétés des opérateurs angle-bornés et $n$-cycliquement monotones," Israel Journal of Mathematics, vol. 26, no. 2, pp. 137-150, 1977.

[7] H.-K. Xu, "Averaged mappings and the gradient-projection algorithm," Journal of Optimization Theory and Applications, vol. 150, no. 2, pp. 360-378, 2011.

[8] L.-C. Ceng, Q. H. Ansari, and J.-C. Yao, "An extragradient method for solving split feasibility and fixed point problems," Computers \& Mathematics with Applications, vol. 64, no. 4, pp. 633-642, 2012.

[9] L.-C. Ceng, Q. H. Ansari, and J.-C. Yao, "Relaxed extragradient methods for finding minimum-norm solutions of the split feasibility problem," Nonlinear Analysis: Theory, Methods \& Applications, vol. 75, no. 4, pp. 2116-2125, 2012.

[10] J. L. Lions, Quelques Méthodes de Résolution des Problèmes aux Limites Non Linéaires, Dunod, Paris, France, 1969.

[11] R. Glowinski, Numerical Methods for Nonlinear Variational Problems, Springer, New York, NY, USA, 1984.

[12] W. Takahashi, Nonlinear Functional Analysis, Yokohama Publishers, Yokohama, Japan, 2000.

[13] J. T. Oden, Quantitative Methods on Nonlinear Mechanics, Prentice Hall, Englewood Cliffs, NJ, USA, 1986.

[14] E. Zeidler, Nonlinear Functional Analysis and Its Applications, Springer, New York, NY, USA, 1985.

[15] G. M. Korpelevič, "An extragradient method for finding saddle points and for other problems," Ekkonomika i Matematicheskie Metody, vol. 12, no. 4, pp. 747-756, 1976. 
[16] N. Nadezhkina and W. Takahashi, "Weak convergence theorem by an extragradient method for nonexpansive mappings and monotone mappings," Journal of Optimization Theory and Applications, vol. 128, no. 1, pp. 191-201, 2006.

[17] L.-C. Zeng and J.-C. Yao, "Strong convergence theorem by an extragradient method for fixed point problems and variational inequality problems," Taiwanese Journal of Mathematics, vol. 10, no. 5, pp. 1293-1303, 2006.

[18] N. Nadezhkina and W. Takahashi, "Strong convergence theorem by a hybrid method for nonexpansive mappings and Lipschitz-continuous monotone mappings," SIAM Journal on Optimization, vol. 16, no. 4, pp. 1230-1241, 2006.

[19] L.-C. Ceng and J.-C. Yao, "An extragradient-like approximation method for variational inequality problems and fixed point problems," Applied Mathematics and Computation, vol. 190, no. 1, pp. 205-215, 2007.

[20] K. Goebel and W. A. Kirk, "A fixed point theorem for asymptotically nonexpansive mappings," Proceedings of the American Mathematical Society, vol. 35, pp. 171-174, 1972.

[21] R. E. Bruck, T. Kuczumow, and S. Reich, "Convergence of iterates of asymptotically nonexpansive mappings in Banach spaces with the uniform Opial property," Colloquium Mathematicum, vol. 65 , no. 2, pp. 169-179, 1993.

[22] T.-H. Kim and H.-K. Xu, "Convergence of the modified Mann's iteration method for asymptotically strict pseudo-contractions," Nonlinear Analysis: Theory, Methods \& Applications, vol. 68, no. 9, pp. 2828-2836, 2008.

[23] D. R. Sahu, H.-K. Xu, and J.-C. Yao, "Asymptotically strict pseudocontractive mappings in the intermediate sense," Nonlinear Analysis: Theory, Methods \& Applications, vol. 70, no. 10, pp. 3502-3511, 2009.

[24] S. Huang, "Hybrid extragradient methods for asymptotically strict pseudo-contractions in the intermediate sense and variational inequality problems," Optimization, vol. 60, no. 6, pp. 739-754, 2011.

[25] L.-C. Ceng and J.-C. Yao, "Strong convergence theorems for variational inequalities and fixed point problems of asymptotically strict pseudocontractive mappings in the intermediate sense," Acta Applicandae Mathematicae, vol. 115, no. 2, pp. 167191, 2011.

[26] Y. Yao, H. Zhou, and Y.-C. Liou, "Weak and strong convergence theorems for an asymptotically $k$-strict pseudo-contraction and a mixed equilibrium problem," Journal of the Korean Mathematical Society, vol. 46, no. 3, pp. 561-576, 2009.

[27] S. Takahashi and W. Takahashi, "Strong convergence theorem for a generalized equilibrium problem and a nonexpansive mapping in a Hilbert space," Nonlinear Analysis: Theory, Methods \& Applications, vol. 69, no. 3, pp. 1025-1033, 2008.

[28] L. C. Ceng, A. Petruşel, and J. C. Yao, "Iterative approaches to solving equilibrium problems and fixed point problems of infinitely many nonexpansive mappings," Journal of Optimization Theory and Applications, vol. 143, no. 1, pp. 37-58, 2009.

[29] L.-C. Ceng and J.-C. Yao, "Approximate proximal methods in vector optimization," European Journal of Operational Research, vol. 183, no. 1, pp. 1-19, 2007.

[30] L.-C. Ceng, Q. H. Ansari, and J.-C. Yao, "An extragradient method for solving split feasibility and fixed point problems," Computers \& Mathematics with Applications, vol. 64, no. 4, pp. 633-642, 2012.

[31] L.-C. Ceng, Q. H. Ansari, and J.-C. Yao, "Relaxed extragradient methods for finding minimum-norm solutions of the split feasibility problem," Nonlinear Analysis: Theory, Methods \& Applications, vol. 75, no. 4, pp. 2116-2125, 2012.

[32] C. Byrne, "A unified treatment of some iterative algorithms in signal processing and image reconstruction," Inverse Problems, vol. 20, no. 1, pp. 103-120, 2004.

[33] P. L. Combettes, "Solving monotone inclusions via compositions of nonexpansive averaged operators," Optimization, vol. 53, no. 5-6, pp. 475-504, 2004.

[34] J. Górnicki, "Weak convergence theorems for asymptotically nonexpansive mappings in uniformly convex Banach spaces," Commentationes Mathematicae Universitatis Carolinae, vol. 30, no. 2, pp. 249-252, 1989.

[35] G. Marino and H.-K. Xu, "Weak and strong convergence theorems for strict pseudo-contractions in Hilbert spaces," Journal of Mathematical Analysis and Applications, vol. 329, no. 1, pp. 336-346, 2007.

[36] H. K. Xu, "Existence and convergence for fixed points of mappings of asymptotically nonexpansive type," Nonlinear Analysis: Theory, Methods \& Applications, vol. 16, no. 12, pp. 1139-1146, 1991.

[37] M. O. Osilike, S. C. Aniagbosor, and B. G. Akuchu, "Fixed points of asymptotically demicontractive mappings in arbitrary Banach spaces," Panamerican Mathematical Journal, vol. 12, no. 2, pp. 77-88, 2002.

[38] K.-K. Tan and H. K. Xu, "Approximating fixed points of nonexpansive mappings by the Ishikawa iteration process," Journal of Mathematical Analysis and Applications, vol. 178, no. 2, pp. 301-308, 1993.

[39] K. Geobel and W. A. Kirk, Topics on Metric Fixed-Point Theory, Cambridge University Press, Cambridge, UK, 1990.

[40] R. T. Rockafellar, "On the maximality of sums of nonlinear monotone operators," Transactions of the American Mathematical Society, vol. 149, pp. 75-88, 1970.

[41] L.-C. Ceng and J.-C. Yao, "A hybrid iterative scheme for mixed equilibrium problems and fixed point problems," Journal of Computational and Applied Mathematics, vol. 214, no. 1, pp. 186201, 2008.

[42] C. Martinez-Yanes and H.-K. Xu, "Strong convergence of the CQ method for fixed point iteration processes," Nonlinear Analysis: Theory, Methods \& Applications, vol. 64, no. 11, pp. 2400-2411, 2006. 


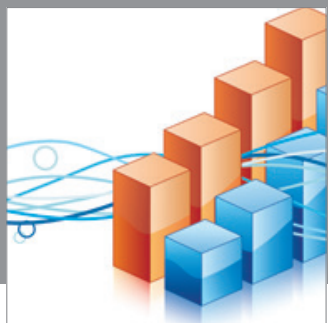

Advances in

Operations Research

mansans

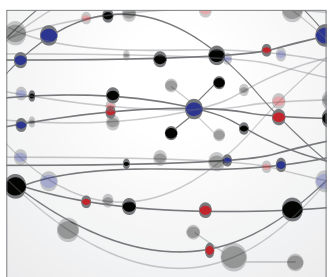

The Scientific World Journal
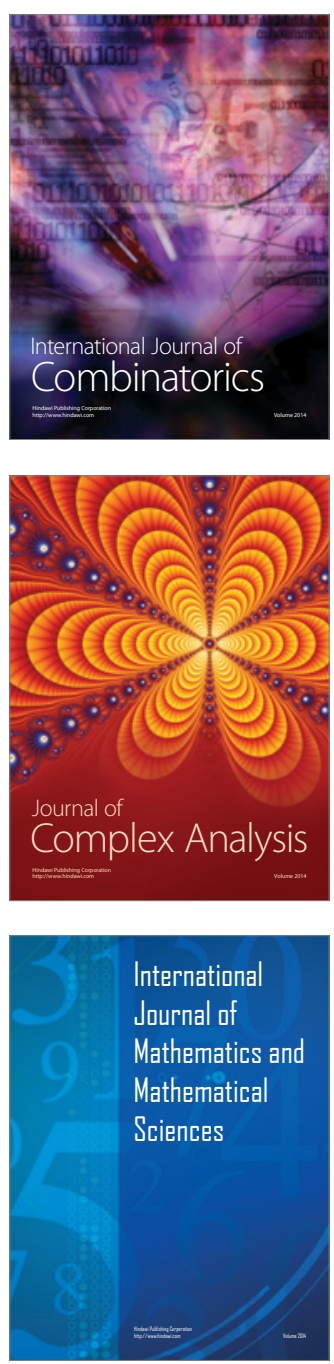
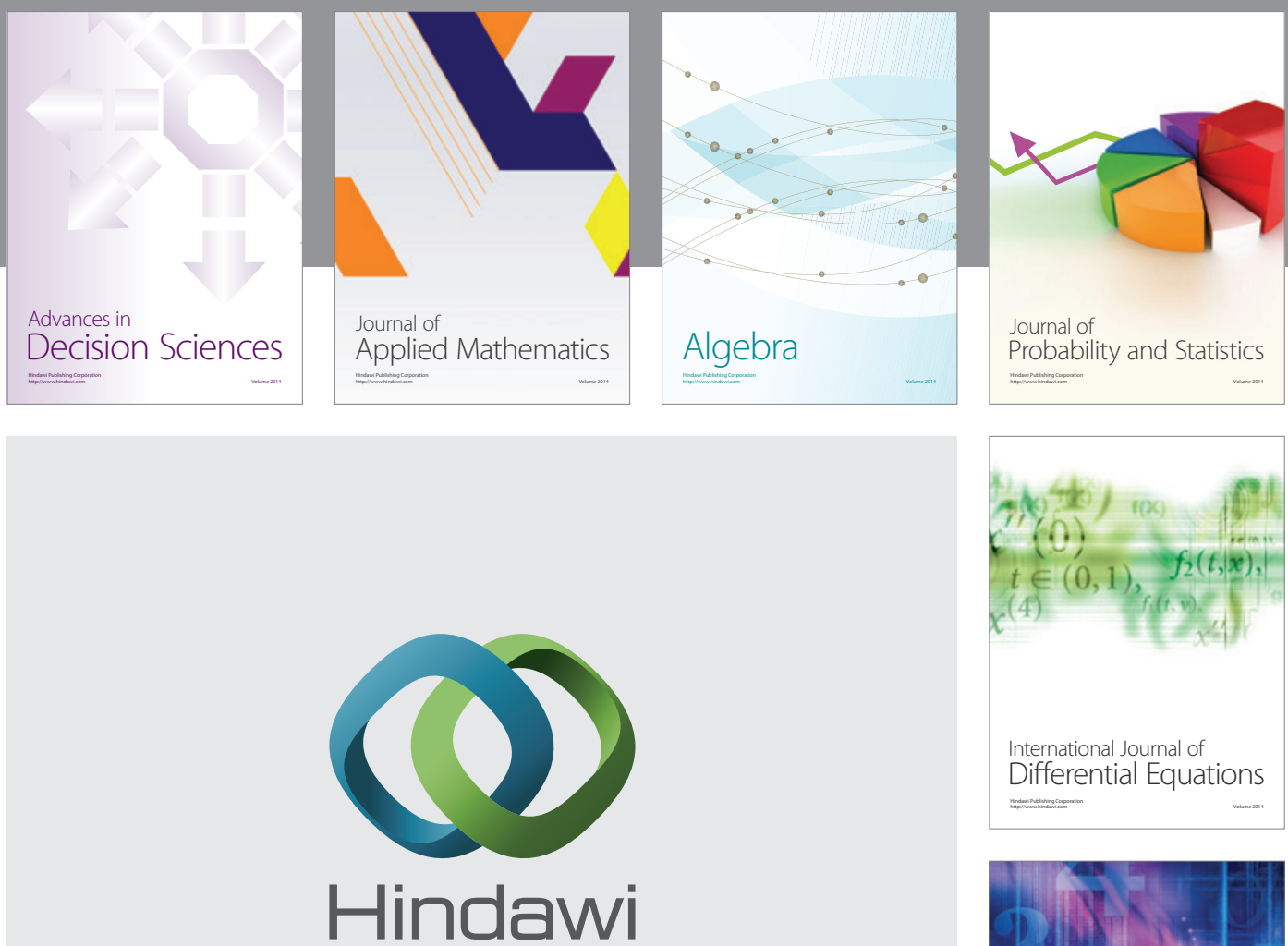

Submit your manuscripts at http://www.hindawi.com
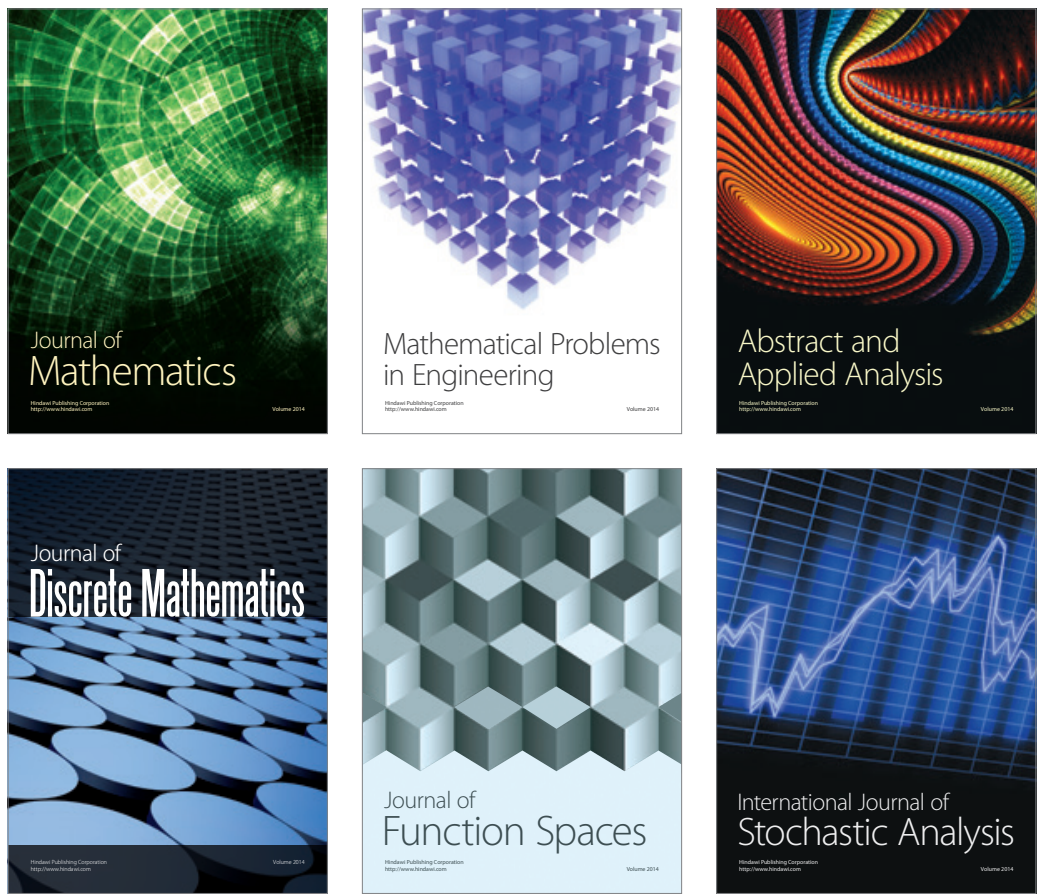

Journal of

Function Spaces

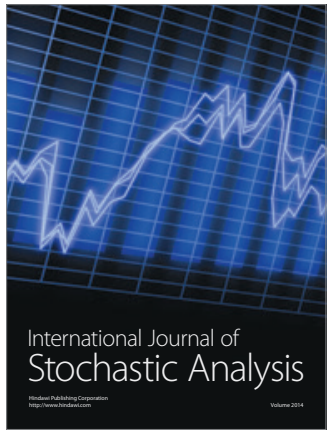

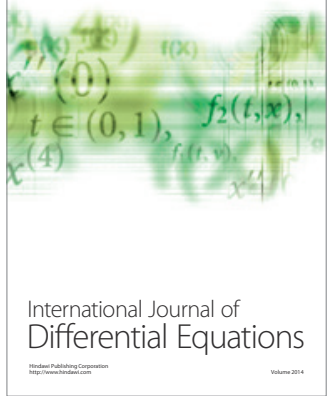
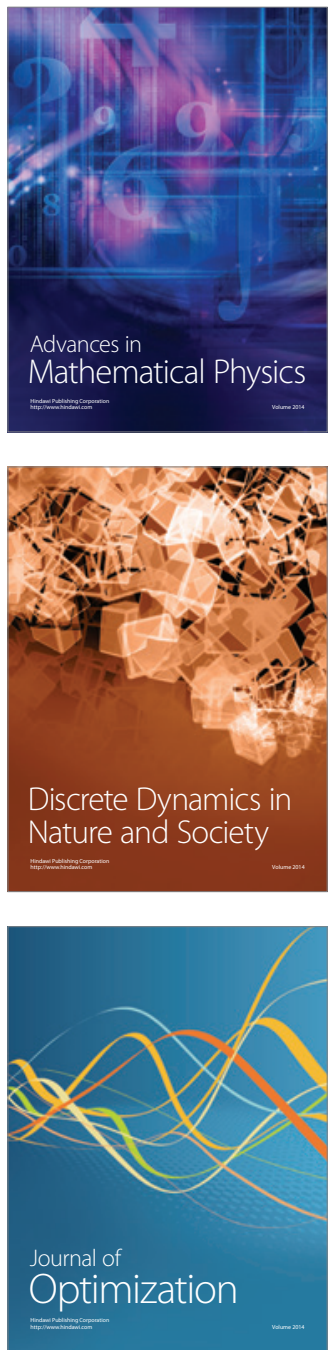